\title{
Stability of finite difference schemes for hyperbolic initial boundary value problems II
}

\author{
JEAN-FRANÇOIS COULOMBEL
}

\begin{abstract}
We study the stability of finite difference schemes for hyperbolic initial boundary value problems in one space dimension. Assuming $\ell^{2}$-stability for the discretization of the hyperbolic operator as well as a geometric regularity condition, we show that the uniform Kreiss-Lopatinskii condition yields strong stability for the discretized initial boundary value problem. The present work extends the results of $[4,7]$ to the widest possible class of finite difference schemes by dropping the technical assumptions of our former work [4]. We give some new examples of numerical schemes for which our results apply.
\end{abstract}

Mathematics Subject Classification (2010): 65M12 (primary); 65M06, 35L50 (secondary).

\section{Introduction}

The aim of this article is to study the stability of finite difference approximations for hyperbolic initial boundary value problems. This problem was addressed in the fundamental contributions [7, 8] for one-dimensional problems, and in [12] for multidimensional problems. The main results of these articles characterize stability in terms of a spectral condition, the so-called uniform Kreiss-Lopatinskii condition. It is not so hard to see that the latter is a necessary condition for stability of the finite difference approximation. The difficult part of the theory is to show that it is also a sufficient condition. The approach of $[7,8,12]$ is similar to the works $[9,15]$ which were devoted to the analogous characterization for hyperbolic partial differential equations. We also refer to $[2,3]$ for a detailed description of the theory.

In the works $[7,12]$ some dissipation assumptions are made on the finite difference schemes, which restricts the class of numerical schemes to which the theory applies. Moreover the underlying partial differential equation that we approximate is not dissipative. Therefore the result we aim at is to prove that the uniform KreissLopatinskii condition is sufficient for stability while considering the widest possible class of numerical schemes. In particular we wish the theory to cover the case of

Research of the author was supported by the Agence Nationale de la Recherche, contract ANR08-JCJC-0132-01.

Received September 9, 2009; accepted in revised form January 8, 2010. 
numerical schemes with a very low dissipation. This is of particular relevance in several space dimensions where some problems are only weakly well-posed and numerical approximations should reproduce this feature: dissipation should not damp weak stability.

In one space dimension, the generalization of the results of [7] was initiated in our former work [4]. However, the main result of [4] could not cover all possible situations due to some technical restrictions which we did not fully understand at that time. In this article we give some examples of numerical schemes that do not enter the framework of [4], nor do they enter the framework of [7]. This makes a generalization of [4] necessary in order to cover all possible cases. The present article generalizes the results of [4] and gives an optimal characterization of stability. Our result is optimal in the following sense. For finite difference schemes, the characterization of stability by means of the uniform Kreiss-Lopatinskii condition relies on a suitable discrete block structure. The only assumption that we make here is that the discrete block structure is satisfied. On the opposite, the technical assumptions of $[4,7]$ restricted either the size or the form of the blocks. The present article considers all possible blocks, including some for which new symmetrizers are required. Our main contribution is the construction of symmetrizers in all possible cases, which, in our opinion, shows the full power of Kreiss' approach. We thus complete the stability theory for one-dimensional finite difference approximations. We postpone the extension of our results for multidimensional problems to a future work. Since the stability theory for numerical schemes is closely linked to the stability theory for partial differential equations, we hope that our new construction of symmetrizers may be useful in other contexts.

No previous knowledge of our work [4] is required since the results are recalled -though without proof- when needed.

\section{Notation}

Throughout this article, we use the notation

$$
\begin{array}{ll}
\mathcal{U}:=\{\zeta \in \mathbb{C},|\zeta|>1\}, & \overline{\mathcal{U}}:=\{\zeta \in \mathbb{C},|\zeta| \geq 1\} \\
\mathbb{D}:=\{\zeta \in \mathbb{C},|\zeta|<1\}, & \mathbb{S}^{1}:=\{\zeta \in \mathbb{C},|\zeta|=1\} .
\end{array}
$$

We let $\mathcal{M}_{d, p}(\mathbb{K})$ denote the set of $d \times p$ matrices with entries in $\mathbb{K}=\mathbb{R}$ or $\mathbb{C}$, and we use the notation $\mathcal{M}_{d}(\mathbb{K})$ when $p=d$. The group of invertible matrices of size $d$ is denoted $G l_{d}(\mathbb{K})$. If $M \in \mathcal{M}_{d}(\mathbb{C}), \operatorname{sp}(M)$ denotes the spectrum of $M$ while $M^{*}$ denotes the conjugate transpose of $M$. The matrix $\left(M+M^{*}\right) / 2$ is called the real part of $M$ and is denoted $\operatorname{Re}(M)$. The real vector space of Hermitian matrices of size $d$ is denoted $\mathcal{H}_{d}$. The vector space of real symmetric matrices of size $d$ is denoted $\mathcal{S}_{d}$. We let $I_{d}$ denote the identity matrix of size $d$, without mentioning the dimension when no confusion is possible. If $H_{1}, H_{2} \in \mathcal{H}_{d}$, the notation $H_{1} \geq H_{2}$ is used when the inequality $x^{*}\left(H_{1}-H_{2}\right) x \geq 0$ holds for all $x \in \mathbb{C}^{d}$. The norm of a vector $x \in \mathbb{C}^{d}$ is $|x|:=\left(x^{*} x\right)^{1 / 2}$. The corresponding norm on $\mathcal{M}_{d}(\mathbb{C})$ is denoted 
$\|\cdot\|$. Eventually, we let $\ell^{2}$ denote the set of square integrable sequences, without mentioning the indices of the sequences. Sequences may be valued in $\mathbb{C}^{k}$ for some integer $k$.

\section{Main result}

We consider a hyperbolic initial boundary value problem in one space dimension

$$
\begin{cases}\partial_{t} u+A \partial_{x} u=F(t, x), & (t, x) \in \mathbb{R}^{+} \times \mathbb{R}^{+}, \\ B u(t, 0)=g(t), & t \in \mathbb{R}^{+}, \\ u(0, x)=f(x), & x \in \mathbb{R}^{+},\end{cases}
$$

where $A \in \mathcal{M}_{N}(\mathbb{R})$ is diagonalizable with real eigenvalues, and $B \in \mathcal{M}_{N_{+}, N}(\mathbb{R})$ with $N_{+}$the number of positive eigenvalues of $A$ counted with their multiplicity. We introduce a finite difference discretization of (2.1). Let $\Delta x, \Delta t>0$ denote a space and a time step where the ratio $\lambda:=\Delta t / \Delta x$ is a fixed positive constant, and let $p, q, r, s$ be some integers. The solution to (2.1) is approximated by a sequence $\left(U_{j}^{n}\right)$ defined for $n \in \mathbb{N}$, and $j \in 1-r+\mathbb{N}$. For $j=1-r, \ldots, 0, U_{j}^{n}$ approximates the trace $u(n \Delta t, 0)$ on the boundary $\{x=0\}$, and possibly the trace of normal derivatives. The boundary meshes $[j \Delta x,(j+1) \Delta x[, j=1-r, \ldots, 0$, shrink to $\{0\}$ as $\Delta x$ tends to 0 , so the formal continuous limit problem as $\Delta x$ tends to 0 is set on the half-line $\mathbb{R}^{+}$as for (2.1).

We consider finite difference approximations of (2.1) that read ${ }^{1}$

$$
\begin{cases}U_{j}^{n+1}=\sum_{\sigma=0}^{s} Q_{\sigma} U_{j}^{n-\sigma}+\Delta t F_{j}^{n}, & j \geq 1, \quad n \geq s, \\ U_{j}^{n+1}=\sum_{\sigma=-1}^{s} B_{j, \sigma} U_{1}^{n-\sigma}+g_{j}^{n+1}, & j=1-r, \ldots, 0, \quad n \geq s, \\ U_{j}^{n}=f_{j}^{n}, & j \geq 1-r, \quad n=0, \ldots, s,\end{cases}
$$

where the shift operators $Q_{\sigma}$ and $B_{j, \sigma}$ are given by

$$
Q_{\sigma}:=\sum_{\ell=-r}^{p} A_{\ell, \sigma} T^{\ell}, \quad B_{j, \sigma}:=\sum_{\ell=0}^{q} B_{\ell, j, \sigma} T^{\ell}, \quad T^{\ell} U_{k}^{m}:=U_{k+\ell}^{m} .
$$

In (2.3), all matrices $A_{\ell, \sigma}, B_{\ell, j, \sigma}$ belong to $\mathcal{M}_{N}(\mathbb{R})$. They may depend on $\lambda$ but are independent of $\Delta t$. We keep $\Delta t$ as a free small parameter while $\Delta x$ is given by the relation $\Delta x=\Delta t / \lambda$.

${ }^{1}$ We do not focus here on the construction of such approximations and refer the reader to [6] for some discretized boundary conditions. 
We recall the following definition:

Definition 2.1 (Strong stability [7]). The finite difference approximation (2.2) is said to be strongly stable if there exists a constant $C_{0}$ such that for all $\gamma>0$ and all $\Delta t \in] 0,1]$, the solution $\left(U_{j}^{n}\right)$ to $(2.2)$ with vanishing initial data $\left(f^{0}=\cdots=\right.$ $\left.f^{s}=0\right)$ satisfies the estimate

$$
\begin{gathered}
\frac{\gamma}{\gamma \Delta t+1} \sum_{n \geq s+1} \sum_{j \geq 1-r} \Delta t \Delta x \mathrm{e}^{-2 \gamma n \Delta t}\left|U_{j}^{n}\right|^{2}+\sum_{n \geq s+1} \sum_{j=1-r}^{0} \Delta t \mathrm{e}^{-2 \gamma n \Delta t}\left|U_{j}^{n}\right|^{2} \\
\leq C_{0}\left\{\frac{\gamma \Delta t+1}{\gamma} \sum_{n \geq s} \sum_{j \geq 1} \Delta t \Delta x \mathrm{e}^{-2 \gamma(n+1) \Delta t}\left|F_{j}^{n}\right|^{2}\right. \\
\left.+\sum_{n \geq s+1} \sum_{j=1-r}^{0} \Delta t \mathrm{e}^{-2 \gamma n \Delta t}\left|g_{j}^{n}\right|^{2}\right\} .
\end{gathered}
$$

For later use, we introduce the symbol (or amplification matrix) associated with the discretization of the hyperbolic operator

$$
\begin{gathered}
\forall \kappa \in \mathbb{C} \backslash\{0\}, \quad \mathcal{A}(\kappa):=\left(\begin{array}{cccc}
\widehat{Q_{0}}(\kappa) & \ldots & \ldots & \widehat{Q_{s}}(\kappa) \\
I & 0 & \ldots & 0 \\
0 & \ddots & \ddots & \vdots \\
0 & 0 & I & 0
\end{array}\right) \in \mathcal{M}_{N(s+1)}(\mathbb{C}), \\
\widehat{Q_{\sigma}}(\kappa):=\sum_{\ell=-r}^{p} \kappa^{\ell} A_{\ell, \sigma}
\end{gathered}
$$

The uniform power boundedness of $\mathcal{A}(\kappa)$ for $\kappa \in \mathbb{S}^{1}$ is a necessary and sufficient condition for the $\ell^{2}$-stability of the discretized Cauchy problem, see [5, Chapter III.1] or [6, Chapter 5]. The aim of this article is to give necessary and/or sufficient conditions on the symbol (2.4) and on the boundary conditions in (2.2) so that the scheme (2.2) is strongly stable in the sense of Definition 2.1.

The resolvent equation is formally obtained from (2.2) by applying a Laplace transform in time, see [7]. It reads

$$
\left\{\begin{array}{l}
w_{j}-\sum_{\sigma=0}^{s} z^{-\sigma-1} Q_{\sigma} w_{j}=F_{j}, \quad j \geq 1, \\
w_{j}-\sum_{\sigma=-1}^{s} z^{-\sigma-1} B_{j, \sigma} w_{1}=g_{j}, \quad j=1-r, \ldots, 0
\end{array}\right.
$$

where $z \in \mathcal{U},\left(F_{j}\right) \in \ell^{2}$, and $g_{1-r}, \ldots, g_{0} \in \mathbb{C}^{N}$. It is convenient to rewrite the resolvent equation (2.5) as an evolution equation for the sequence $\left(w_{j}\right)$. Assumption 
2.2 below is crucial in order to achieve this transformation. For $\ell=-r, \ldots, p$, let us therefore define the matrices

$$
\forall z \in \mathbb{C} \backslash\{0\}, \quad \mathbb{A}_{\ell}(z):=\delta_{\ell, 0} I-\sum_{\sigma=0}^{s} z^{-\sigma-1} A_{\ell, \sigma},
$$

where $\delta_{\ell_{1}, \ell_{2}}$ is the Kronecker symbol. Then as in [7], we make the following assumption:

Assumption 2.2 (Discrete noncharacteristic boundary). The matrices $\mathbb{A}_{-r}(z)$ and $\mathbb{A}_{p}(z)$ are invertible for all $z \in \overline{\mathcal{U}}$, or equivalently for all $z$ in some open neighborhood $\mathcal{V}$ of $\overline{\mathcal{U}}$.

We first consider the case $q<p$. In that case, all the $w_{j}$ 's involved in the boundary conditions for the resolvent equation (2.5) are coordinates of the augmented vector ${ }^{2} W_{1}:=\left(w_{p}, \ldots, w_{1-r}\right)$. Using Assumption 2.2, we can define a matrix $\mathbb{M}(z)$ that is holomorphic on some open neighborhood $\mathcal{V}$ of $\overline{\mathcal{U}}$

$$
\begin{aligned}
\forall z \in \mathcal{V}, \quad \mathbb{M}(z) & :=\left(\begin{array}{ccccc}
-\mathbb{A}_{p}(z)^{-1} \mathbb{A}_{p-1}(z) & \ldots & \ldots & -\mathbb{A}_{p}(z)^{-1} \mathbb{A}_{-r}(z) \\
I & 0 & \ldots & 0 \\
0 & \ddots & \ddots & \vdots \\
0 & 0 & I & 0
\end{array}\right) \\
& \in \mathcal{M}_{N(p+r)}(\mathbb{C}) .
\end{aligned}
$$

Using (2.3) and (2.6), we can rewrite the resolvent equation (2.5) as an induction relation for the augmented vector $W_{j}:=\left(w_{j+p-1}, \ldots, w_{j-r}\right)$. This induction relation reads

$$
\left\{\begin{array}{l}
W_{j+1}=\mathbb{M}(z) W_{j}+\widetilde{F}_{j}, \quad j \geq 1, \\
B(z) W_{1}=g,
\end{array}\right.
$$

with some suitable source terms $\left(\widetilde{F_{j}}\right), g$. It is easy to check that the matrix $B(z) \in$ $\mathcal{M}_{N r, N(p+r)}$ that encodes the boundary conditions in (2.8) depends holomorphically on $z \in \mathbb{C} \backslash\{0\}$ and has maximal rank $N r$ for all $z$. The exact expression of the matrix $B(z)$ can be easily obtained from (2.5) and (2.3) but is not very relevant here so we omit it.

Let us now treat the case $q \geq p$. In that case, we can still write the resolvent equation under the form of a one-step induction relation up to defining $W_{j}:=$ $\left(w_{j+q}, \ldots, w_{j-r}\right), j \geq 1$, and

$$
\mathbb{M}(z):=\left(\begin{array}{cccccc}
-\mathbb{A}_{p}(z)^{-1} \mathbb{A}_{p-1}(z) & \ldots & -\mathbb{A}_{p}(z)^{-1} \mathbb{A}_{-r}(z) & 0 & \ldots & 0 \\
I & 0 & \ldots & 0 & & 0 \\
0 & & \ddots & & & \\
& & & & & \\
0 & & \ldots & 0 & I & 0
\end{array}\right) \in \mathcal{M}_{N(q+r+1)}(\mathbb{C}) .
$$

${ }^{2}$ Vectors are written indifferently in rows or columns to simplify the writing. 
The definition of $B(z) \in \mathcal{M}_{N r, N(q+r+1)}$ varies from the previous case but this matrix keeps a maximal rank $N r$ for all $z$ and is still holomorphic on $\mathbb{C} \backslash\{0\}$. This equivalent form of the resolvent equation varies from what was done in [7, page 672]. In our approach, we can easily verify that the matrix $B(z)$ has maximal rank for all $z \in \overline{\mathcal{U}}$. This is important in view of the so-called uniform Kreiss-Lopatinskii condition defined below.

For simplicity, we shall deal from now on with the case $q<p$ but our proofs can be easily extended to the case $q \geq p$.

Theorem 2.3. Let Assumption 2.2 be satisfied. Assume moreover that the symbol $\mathcal{A}$ defined by (2.4) satisfies the two following conditions

- Uniform power boundedness: there exists a constant $C_{1}>0$ such that for all $\kappa \in \mathbb{S}^{1}$ and all $n \in \mathbb{N},\left\|\mathcal{A}(\kappa)^{n}\right\| \leq C_{1}$,

- Geometric regularity of eigenvalues in $\mathbb{S}^{1}$ : if $\underline{\kappa} \in \mathbb{S}^{1}$ and $\underline{z} \in \mathbb{S}^{1} \cap \operatorname{sp}(\mathcal{A}(\underline{\kappa}))$ has algebraic multiplicity $\underline{\alpha}$, then there exist some functions $\lambda_{1}(\kappa), \ldots, \lambda_{\alpha}(\kappa)$ that are holomorphic in a neighborhood $\mathcal{W}$ of $\underline{\kappa}$ in $\mathbb{C}$ and that satisfy

$$
\lambda_{1}(\underline{\kappa})=\cdots=\lambda_{\underline{\alpha}}(\underline{\kappa})=\underline{z}, \quad \operatorname{det}(z I-\mathcal{A}(\kappa))=\vartheta(\kappa, z) \prod_{j=1}^{\underline{\alpha}}\left(z-\lambda_{j}(\kappa)\right),
$$

with $\vartheta$ a holomorphic function of $(\kappa, z)$ in some neighborhood of $(\underline{\kappa}, \underline{z})$ such that $\vartheta(\underline{\kappa}, \underline{z}) \neq 0$. Furthermore, there exist some vectors $E_{1}(\kappa), \ldots, E_{\underline{\alpha}}(\kappa) \in$ $\mathbb{C}^{N(s+1)}$ that depend holomorphically on $\kappa \in \mathcal{W}$, that are linearly independent for all $\kappa \in \mathcal{W}$, and that satisfy

$$
\forall \kappa \in \mathcal{W}, \quad \forall j=1, \ldots, \underline{\alpha}, \quad \mathcal{A}(\kappa) E_{j}(\kappa)=\lambda_{j}(\kappa) E_{j}(\kappa)
$$

For $z \in \mathcal{U}$, we let $\mathbb{E}^{s}(z)$ denote the generalized eigenspace associated with eigenvalues of $\mathbb{M}(z)$ in $\mathbb{D}$. Then $\mathbb{E}^{s}(z)$ has constant dimension $N$ for all $z \in \mathcal{U}$ and $\mathbb{E}^{s}$ defines a holomorphic vector bundle over $\mathcal{U}$. This vector bundle can be extended continuously in a unique way over $\overline{\mathcal{U}}$. We let $\mathbb{E}^{s}(z)$ denote this continuous extension for $z \in \mathbb{S}^{1}(=\partial \mathcal{U})$.

In addition to all previous assumptions, assume that for all $z \in \overline{\mathcal{U}}$ we have $\mathbb{E}^{s}(z) \cap \operatorname{Ker} B(z)=\{0\}$. In what follows this condition is referred to as the uniform Kreiss-Lopatinskii condition. Then the scheme (2.2) is strongly stable in the sense of Definition 2.1.

Theorem 2.3 shows that proving stability for the numerical scheme (2.2) follows the same path as when one studies multidimensional hyperbolic initial boundary value problems, see for instance [2,3,9, 15] and above all [11, Appendix C]. More precisely, we first make the assumption that the boundary is noncharacteristic. This is Assumption 2.2. Then two assumptions are made on the discretized hyperbolic operator. 
- The uniform power boundedness of the matrices $\mathcal{A}(\kappa)$ is a stability assumption for the discretized Cauchy problem. This condition plays the same role as hyperbolicity for the continuous problem. Let us observe that for all $\kappa \in \mathbb{S}^{1}$, the eigenvalues of the matrix $\mathcal{A}(\kappa)$ necessarily belong to $\mathbb{D} \cup \mathbb{S}^{1}$. Moreover, eigenvalues of $\mathcal{A}(\kappa)$ that belong to $\mathbb{S}^{1}$ are semi-simple.

- The geometric regularity assumption makes the behavior of eigenvalues and eigenvectors precise near a point $\underline{\kappa} \in \mathbb{S}^{1}$ where the spectrum of $\mathcal{A}(\underline{\kappa})$ meets $\mathbb{S}^{1}$. This assumption is similar to the geometric regularity condition that characterizes the block structure for continuous problems, see [11, Appendix C]. Let us observe that the matrix

$$
\left(\begin{array}{cc}
1+\Upsilon(\kappa) & \Upsilon(\kappa) \\
0 & 1+\Upsilon(\kappa)
\end{array}\right), \quad \Upsilon(\kappa):=\frac{\left(\kappa-\kappa^{-1}\right)^{2}}{4},
$$

is holomorphic with respect to $\kappa \in \mathbb{C} \backslash\{0\}$, and is uniformly power bounded for $\kappa \in \mathbb{S}^{1}$. However 1 is not a geometrically regular eigenvalue near $\underline{\kappa}=1$. Geometric regularity does not automatically follow from uniform power boundedness.

The assumptions on $\mathcal{A}$ enable us to extend the stable bundle of $\mathbb{M}$ from $\mathcal{U}$ to $\overline{\mathcal{U}}$ in a unique way. As in the theory for multidimensional hyperbolic initial boundary value problems, this result is independent of the boundary conditions that are considered in the scheme (2.2). Once this first result is known, then strong stability for (2.2) is encoded in the so-called uniform Kreiss-Lopatinskii condition. This condition can be equivalently formulated as a determinant condition by choosing a basis of $\mathbb{E}^{s}(z)$ and a basis of $\operatorname{Ker} B(z)$. Let us observe that this characterization of strong stability makes sense only when $B(z)$ has maximal rank for all $z \in \overline{\mathcal{U}}$, otherwise the dimensions of $\mathbb{E}^{S}(z)$ and $\operatorname{Ker} B(z)$ do not match.

Compared to the previous works $[4,7]$, Theorem 2.3 drops the technical assumptions that were made on the symbol $\mathcal{A}$, see [7, Assumptions 5.2 and 5.4] or the less restrictive conditions (i), (ii), (iii) of [4, Theorem 2.7]. In particular, Theorem 2.3 makes precise the structural assumptions that are needed to prove the continuous extension of the stable bundle of $\mathbb{M}$. As a corollary of our analysis, if $\mathbb{M}$ satisfies the discrete block structure recalled in Theorem 3.1 below, then the stable bundle of $\mathbb{M}$ extends continuously from $\mathcal{U}$ to $\overline{\mathcal{U}}$.

Eventually, we observe that Theorem 2.3 is optimal if one wishes to characterize strong stability by the uniform Kreiss-Lopatinskii condition. More precisely, when the energy method is not available, showing strong stability for (2.2) requires the construction of a so-called Kreiss symmetrizer. Such construction relies on the discrete block structure which is recalled in Theorem 3.1. As proved in [4, Theorem 2.4], the discrete block structure is satisfied if and only if the structural assumptions of Theorem 2.3 hold for $\mathcal{A}$. Consequently, one could rephrase Theorem 2.3 by assuming only that the discrete block structure holds for $\mathbb{M}$.

The rest of this article is organized as follows: in Section 3, we make some preliminary reductions which show that the proof of Theorem 2.3 reduces to the 
construction of a so-called $K$-symmetrizer. The arguments of Section 3 use some results of our former work [4] which are recalled for the reader's convenience. Then in Sections 4-8, we give a complete construction of the $K$-symmetrizer. Our results generalize the constructions in $[2,3,9,15]$ which were devoted either to the hyperbolic case with no dissipation or to scalar blocks. Our new construction depends on the size of the block we consider and on its dissipation index. This notion is the crucial novelty compared to [4] and is introduced in Section 4. We are able to construct a $K$-symmetrizer for any dissipative block, while the construction in [4] was restricted to some specific dissipative $2 \times 2$ blocks. Eventually, Section 9 provides with some examples of numerical schemes that produce dissipative blocks. To our knowledge, no existing theory was able to prove strong stability for such schemes.

Some results of matrix theory are used throughout the article, some of which are proved in Appendix A. Eventually, the reader could reasonably ask whether new symmetrizers are really needed to deal with the dissipative blocks considered in this article. At first glance one might hope that the constructions in [9] would work even though they had not been designed for this purpose. This question is discussed in Appendix B. In particular, Appendix B shows that Kreiss' construction does not apply for the dissipative blocks that we consider. This makes our work both new and relevant.

\section{Preliminary reductions}

\subsection{The discrete block structure}

We first recall the following theorem that was proved in our former work [4]:

Theorem 3.1 ([4]). Let Assumption 2.2 be satisfied and assume furthermore that the symbol $\mathcal{A}$ defined by (2.4) satisfies the two conditions stated in Theorem 2.3, that is uniform power boundedness and geometric regularity of eigenvalues in $\mathbb{S}^{1}$.

Then the matrix $\mathbb{M}$ defined by (2.7) satisfies the so-called discrete block structure condition:

1. for all $z \in \mathcal{U}, \operatorname{sp}(\mathbb{M}(z)) \cap \mathbb{S}^{1}=\emptyset$,

2. for all $\underline{z} \in \overline{\mathcal{U}}$, there exists an open neighborhood $\mathcal{O}$ of $\underline{z}$ in $\mathbb{C}$, there exists a holomorphic function $T$ defined on $\mathcal{O}$ with values in $G l_{N(p+r)}(\mathbb{C})$ such that

$$
\forall z \in \mathcal{O}, \quad T(z)^{-1} \mathbb{M}(z) T(z)=\operatorname{diag}\left(M_{1}(z), \ldots, M_{L}(z)\right),
$$

where the number $L$ of diagonal blocks and the size $m_{\ell}$ of each block $M_{\ell}$ do not depend on $z \in \mathcal{O}$, and where each block satisfies one of the following properties:

- there exists $\varepsilon>0$ such that for all $z \in \mathcal{O}, M_{\ell}(z)^{*} M_{\ell}(z) \geq(1+\varepsilon) I$,

- there exists $\varepsilon>0$ such that for all $z \in \mathcal{O}, M_{\ell}(z)^{*} M_{\ell}(z) \leq(1-\varepsilon) I$,

- $m_{\ell}=1, \underline{z}$ and $M_{\ell}(\underline{z})$ belong to $\mathbb{S}^{1}$, and $\underline{z} M_{\ell}^{\prime}(\underline{z}) \overline{M_{\ell}(\underline{z})} \in \overline{\mathbb{R}} \backslash\{0\}$, 
- $m_{\ell} \geq 2, \underline{z} \in \mathbb{S}^{1}$ and $M_{\ell}(\underline{z})$ has the form

$$
M_{\ell}(\underline{z})=\underline{\kappa}_{\ell}\left(\begin{array}{cccc}
1 & 1 & 0 & 0 \\
0 & \ddots & \ddots & 0 \\
\vdots & \ddots & \ddots & 1 \\
0 & \ldots & 0 & 1
\end{array}\right), \quad \underline{\kappa}_{\ell} \in \mathbb{S}^{1} .
$$

Moreover the lower left coefficient $\alpha_{\ell}$ of $M_{\ell}^{\prime}(\underline{z})$ is such that for all $\omega \in \mathbb{C}$ with $\operatorname{Re} \omega>0$, any root $\zeta$ of the equation $\zeta^{m_{\ell}}=\underline{\bar{K}}_{\ell} \alpha_{\ell} \underline{z} \omega$ satisfies $\operatorname{Re} \zeta \neq 0$.

We refer to the blocks $M_{\ell}$ above as being of the first, second, third or fourth type.

The first point in Theorem 3.1 shows that for $z \in \mathcal{U}, \mathbb{M}(z)$ has no eigenvalue on $\mathbb{S}^{1}$. The eigenvalues split in two groups: the stable eigenvalues belonging to $\mathbb{D}$ and the unstable eigenvalues belonging to $\mathcal{U}$. It is then clear that the generalized eigenspace $\mathbb{E}^{s}(z)$ associated with the stable eigenvalues has constant dimension for all $z$ in the connected set $\mathcal{U}$. It varies holomorphically with respect to $z$ because $\mathbb{M}$ depends holomorphically on $z$. The dimension of $\mathbb{E}^{s}(z)$ is computed as in [8, page 706] by letting $z$ tend to infinity, and we obtain that the dimension equals $N r$.

Using the discrete block structure condition, we want to prove that $\mathbb{E}^{s}(z)$ extends by continuity as $z \in \mathcal{U}$ tends to the unit circle $\mathbb{S}^{1}$. Then we also want to prove that the uniform Kreiss-Lopatinskii condition - which is defined after first extending the stable subspace for $z \in \mathbb{S}^{1}$ - implies strong stability for (2.2). Following [10], it turns out that a single argument can give both results at the same time. This requires the introduction of so-called $K$-symmetrizers, which is done in the following paragraph.

\subsection{Symmetrizers and continuity of the stable subspace}

We recall the following terminology that was introduced in [4] for numerical schemes and that is adapted from [10].

Definition 3.2 ( $K$-symmetrizer). Let $\underline{z} \in \overline{\mathcal{U}}$, and let $M$ be a function defined on some neighborhood $\mathcal{O}$ of $\underline{z}$ with values in $\mathcal{M}_{m}(\mathbb{C})$ for some integer $m$. Then $M$ is said to admit a $K$-symmetrizer at $\underline{z}$ if there exists a decomposition

$$
\mathbb{C}^{m}=\underline{\mathbb{E}}^{s} \oplus \underline{\mathbb{E}}^{u},
$$

with associated projectors $\left(\pi^{s}, \pi^{u}\right)$, such that for all $K \geq 1$, there exists a neighborhood $\mathcal{O}_{K}$ of $\underline{z}$, there exists a $\mathcal{C}^{\infty}$ function $S_{K}$ on $\mathcal{O}_{K}$ with values in $\mathcal{H}_{m}$, and there exists a constant $c_{K}>0$ such that the following properties hold for all $z \in \mathcal{O}_{K} \cap \overline{\mathcal{U}}$ :

- $M(z)^{*} S_{K}(z) M(z)-S_{K}(z) \geq c_{K}(|z|-1) /|z| I$,

- for all $W \in \mathbb{C}^{m}, W^{*} S_{K}(z) W \geq K^{2}\left|\underline{\pi}^{u} W\right|^{2}-\left|\underline{\pi}^{s} W\right|^{2}$.

The following result was also proved in [4]. 
Theorem 3.3 ( [4]). Let the assumptions of Theorem 2.3 be satisfied. Assume moreover that the matrix $\mathbb{M}$ defined by (2.7) admits a $K$-symmetrizer at all points of $\overline{\mathcal{U}}$ and that the dimension of the corresponding vector space $\underline{\mathbb{E}}^{s}$ in the decomposition of $\mathbb{C}^{N(p+r)}$ equals $N r$ at all points of $\overline{\mathcal{U}}$.

Then the holomorphic vector bundle $\mathbb{E}^{s}$ defined over $\mathcal{U}$ can be extended in a unique way as a continuous vector bundle over $\overline{\mathcal{U}}$. If moreover the uniform KreissLopatinskii condition holds, then the scheme (2.2) is strongly stable.

In order to prove Theorem 2.3 , we thus only need to construct a $K$-symmetrizer for $\mathbb{M}$ at all points $\underline{z}$ of $\overline{\mathcal{U}}$ with a vector space $\underline{\mathbb{E}}^{s}$ of dimension $N r$. This argument is made more precise in the following paragraph.

\subsection{A sufficient result for proving Theorem 2.3}

Let us first state without proof the following result which is the key point of our work.

Theorem 3.4. Let $\underline{z}, \underline{\kappa} \in \mathbb{S}^{1}$, let $m \geq 2$ be an integer. Let $M$ be a holomorphic function defined on a neighborhood $\mathcal{O}$ of z with values in $\mathcal{M}_{m}(\mathbb{C})$, that satisfies the following three conditions:

- for all $z \in \mathcal{O} \cap \mathcal{U}, \operatorname{sp}(M(z)) \cap \mathbb{S}^{1}=\emptyset$,

- $M(\underline{z})$ has the form

$$
M(\underline{z})=\underline{\kappa}\left(\begin{array}{cccc}
1 & 1 & 0 & 0 \\
0 & \ddots & \ddots & 0 \\
\vdots & \ddots & \ddots & 1 \\
0 & \ldots & 0 & 1
\end{array}\right) .
$$

- The lower left coefficient $\alpha$ of $M^{\prime}(\underline{z})$ is such that for all $\omega \in \mathbb{C}$ with $\operatorname{Re} \omega>0$, any root $\zeta$ of the equation $\zeta^{m}=\underline{\bar{\kappa}} \alpha \underline{z} \omega$ satisfies $\operatorname{Re} \zeta \neq 0$.

Then up to shrinking $\mathcal{O}$, the number of stable eigenvalues of $M(z)$ when $z \in \mathcal{O} \cap \mathcal{U}$ does not depend on $z$. Letting $\mu$ denote this number, $M$ admits a $K$-symmetrizer at $\underline{z}$ with a corresponding vector space $\underline{\mathbb{E}}^{S}$ of dimension $\mu$.

The proof of Theorem 3.4 is detailed in Sections 4-8. We show now why the result of Theorem 3.4 is sufficient for proving Theorem 2.3.

Proof of Theorem 2.3 using Theorem 3.4. Theorem 3.4 shows that blocks of the fourth type in the discrete block structure admit a $K$-symmetrizer. Moreover, we have already shown in our former work [4] that blocks of the first, second and third type admit a $K$-symmetrizer. As a matter of fact, the analysis for these blocks is far easier than for blocks of the fourth type and we refer to [4, page 2863] for the construction of $K$-symmetrizers in this case. For each type of block, the dimension of the corresponding vector space $\mathbb{E}^{s}$ coincides with the number of stable eigenvalues of the block when $z$ belongs to $\overline{\mathcal{O}} \cap \mathcal{U}$. 
We now use the following two results whose proof - which is omitted here relies on some direct applications of Definition 3.2.

Lemma 3.5. Let $\underline{z} \in \overline{\mathcal{U}}$, and let $M_{1}$, respectively $M_{2}$, be a function defined on some neighborhood $\mathcal{O}$ of $\underline{z}$ with values in $\mathcal{M}_{m_{1}}(\mathbb{C})$, respectively $\mathcal{M}_{m_{2}}(\mathbb{C})$, for some integer $m_{1}$, respectively $m_{2}$. Assume that both matrices $M_{1}, M_{2}$ admit a $K$ symmetrizer at $\underline{z}$ with vector spaces $\underline{\mathbb{E}}_{1}^{s}, \underline{\mathbb{E}}_{2}^{s}$ of dimension $\mu_{1}, \mu_{2}$.

Then the block diagonal matrix $\operatorname{diag}\left(M_{1}, M_{2}\right) \in \mathcal{M}_{m_{1}+m_{2}}(\mathbb{C})$ admits a $K$ symmetrizer at $\underline{z}$ with a vector space $\underline{\mathbb{E}}^{s}$ of dimension $\mu_{1}+\mu_{2}$.

Lemma 3.6. Let $\underline{z} \in \overline{\mathcal{U}}$, and let $M$ be a function defined on some neighborhood $\mathcal{O}$ of $z$ with values in $\mathcal{M}_{m}(\mathbb{C})$ for some integer $m$. Assume that there exists a $\mathcal{C}^{\infty}$ function $T$ defined on $\mathcal{O}$ with values in $G l_{m}(\mathbb{C})$ such that $T^{-1} M T$ admits a $K$ symmetrizer at $\underline{z}$ with a vector space $\underline{\mathbb{E}}^{s}$ of dimension $\mu$.

Then $M$ admits a $K$-symmetrizer at $\underline{z}$ with a vector space $\underline{\mathbb{E}}^{s}$ of dimension $\mu$.

Combining Lemma 3.5, Lemma 3.6 and Theorem 3.1 above, we obtain that the matrix $\mathbb{M}$ defined by (2.7) admits a $K$-symmetrizer at all points $\underline{z} \in \overline{\mathcal{U}}$. Moreover, the dimension of the corresponding vector space $\underline{\mathbb{E}}^{s}$ coincides with the number of stable eigenvalues of $\mathbb{M}(z)$ when $z \in \mathcal{U}$ is close to $\underline{z}$. Therefore the dimension of $\underline{\mathbb{E}}^{S}$ equals $N r$. We can apply Theorem 3.3 above, and the result of Theorem 2.3 follows.

The following sections are devoted to the proof of Theorem 3.4, or in other words to the construction of a $K$-symmetrizer for a block of the fourth type.

\section{The dissipation index}

From now on, we consider a matrix $M(z) \in \mathcal{M}_{m}(\mathbb{C})$ satisfying all the assumptions of Theorem 3.4. There is no loss of generality in assuming that the neighborhood $\mathcal{O}$ of $\underline{z}$ is an open disk of center $\underline{z}$ and of radius $r \leq 1$. Consequently $\mathcal{O} \cap \mathcal{U}$ is an open connected set. We first compute the number of stable eigenvalues, that is the number of eigenvalues of $M(z)$ in $\mathbb{D}$ when $z$ belongs to $\mathcal{O} \cap \mathcal{U}$.

For convenience, we introduce the nilpotent matrix $N_{m}$, or $N$ in short when the dimension is clear, which is defined by

$$
N_{m}:=\left(\begin{array}{cccc}
0 & 1 & & 0 \\
\vdots & \ddots & \ddots & \\
\vdots & & \ddots & 1 \\
0 & \cdots & \cdots & 0
\end{array}\right) \in \mathcal{M}_{m}(\mathbb{C})
$$

We warn the reader that $N$ does not refer to the size of the matrices in (2.2) anylonger since from now on we focus on the proof of Theorem 3.4, which is a completely independent result. 


\subsection{The number of stable eigenvalues}

The following result clarifies the number of stable eigenvalues.

Proposition 4.1. Under the assumptions of Theorem 3.4, the number $\mu$ of eigenvalues of $M(z)$ in $\mathbb{D}$ does not depend on $z \in \mathcal{O} \cap \mathcal{U}$. If $m$ is even, we have

$$
\mu=\frac{m}{2}, \quad \frac{\alpha \underline{z}}{\underline{\kappa}} \neq 0 \quad \text { and } \quad(-1)^{m / 2} \operatorname{Re}\left(\frac{\alpha \underline{z}}{\underline{\kappa}}\right) \leq 0 .
$$

If $m$ is odd, then $\alpha \underline{z} / \underline{\kappa} \in \mathbb{R} \backslash\{0\}$ and

$$
\mu= \begin{cases}\frac{m+1}{2} & \text { if }(-1)^{(m-1) / 2} \alpha \underline{z} / \underline{\kappa}<0, \\ \frac{m-1}{2} & \text { if }(-1)^{(m-1) / 2} \alpha \underline{z} / \underline{\kappa}>0 .\end{cases}
$$

Proof of Proposition 4.1. When $z$ belongs to the open connected set $\mathcal{O} \cap \mathcal{U}$, the matrix $M(z)$ has no eigenvalue on the unit circle $\mathbb{S}^{1}$. Consequently, the number of eigenvalues of $M(z)$ in $\mathbb{D}$ does not depend on $z \in \mathcal{O} \cap \mathcal{U}$.

Let us first consider the case where $m$ is even, and $m \equiv 2(4)$. Then we know that for all $\omega \in \mathbb{C}$ with $\operatorname{Re} \omega>0,(\alpha \underline{z} / \underline{\kappa}) \omega$ does not belong to the real nonpositive axis ] $-\infty, 0]$. Otherwise, we could find a purely imaginary $m$-th root of $(\alpha \underline{z} / \underline{\kappa}) \omega$. It is easy to see that the complex number $\alpha \underline{z} / \underline{\kappa}$ satisfies the latter property if and only if it is non-zero and its real part is nonnegative.

In the case where $m$ is even and $m \equiv 0(4)$, we find that for all $\omega \in \mathbb{C}$ with $\operatorname{Re} \omega>0,(\alpha \underline{z} / \underline{\kappa}) \omega$ does not belong to the real nonnegative axis $[0,+\infty[$. This is equivalent to the fact that $\alpha \underline{z} / \underline{\kappa}$ is non-zero and its real part is nonpositive. In both cases, the real part of $(-1)^{m / 2} \alpha \underline{z} / \underline{\kappa}$ is nonpositive.

Let us now consider the case where $m$ is odd. Then for all $\omega \in \mathbb{C}$ with $\operatorname{Re} \omega>$ $0,(\alpha \underline{z} / \underline{\kappa}) \omega$ does not belong to the purely imaginary axis $i \mathbb{R}$. This forces $\alpha \underline{z} / \underline{\kappa}$ to be a non-zero real number.

Let us now compute the number $\mu$ of stable eigenvalues of $M(z)$ when $z \in$ $\mathcal{O} \cap \mathcal{U}$. We define the characteristic polynomial

$$
\forall(z, \kappa) \in \mathcal{O} \times \mathbb{C}, \quad P(z, \kappa):=\operatorname{det}(M(z)-\kappa I) .
$$

When $z=\underline{z}$, we have $P(\underline{z}, \kappa)=(\underline{\kappa}-\kappa)^{m}$. Moreover, the form of $M(\underline{z})$ gives the relation

$$
\frac{\partial P}{\partial z}(\underline{z}, \underline{\kappa})=(-1)^{m+1} \alpha \underline{\kappa}^{m-1} .
$$

Consider $\varepsilon>0$ small enough. Then we compute the number $\mu$ by counting the stable eigenvalues of $M((1+\varepsilon) \underline{z})$. The characteristic polynomial of the matrix $M((1+\varepsilon) \underline{z})$ is $P((1+\varepsilon) \underline{z}, \kappa)$. By using Puiseux expansions theory, for which we refer to [1], the roots of the polynomial $P((1+\varepsilon) \underline{z}, \cdot)$ admit the asymptotic expansion

$$
\kappa_{\ell}(\varepsilon)=\underline{\kappa}\left(1+\varepsilon^{1 / m} \zeta_{\ell}+O\left(\varepsilon^{2 / m}\right)\right), \quad \ell=1, \ldots, m,
$$


where the complex numbers $\zeta_{1}, \ldots, \zeta_{m}$ denote the $m$-th roots of $\alpha \underline{z} / \underline{\kappa}$. Observe that all these $m$-th roots have non-zero real part. Consequently, the number $\mu$ of stable eigenvalues of $M((1+\varepsilon) \underline{z})$ equals the number of $m$-th roots of $\alpha \underline{z} / \underline{\kappa}$ of negative real part (use a Taylor expansion for $\left|\kappa_{\ell}(\varepsilon)\right|$ ).

Let us consider the case where $m$ is even. The $m$-th roots of $\alpha \underline{z} / \underline{\kappa}$ are simple and invariant under the transformation $(\zeta \mapsto-\zeta)$. Therefore $m / 2$ of these roots have positive real part and $m / 2$ have negative real part. Thus $\mu$ equals $m / 2$.

Let us now consider the case where $m$ is odd, and $m \equiv 1$ (4). We know from the analysis above that $\beta:=\alpha \underline{z} / \underline{\kappa}$ is a non-zero real number. Let us first assume that $\beta$ is positive. Then there are exactly $(m-1) / 2$ roots of negative real part among all possible $m$-th roots of $\beta$. If $\beta$ is negative, there are $(m+1) / 2$ roots of negative real part among all possible $m$-th roots of $\beta$. Let us now assume that $m$ is odd, and $m \equiv 3$ (4). If $\beta$ is positive, then there are $(m+1) / 2$ roots of negative real part among all possible $m$-th roots of $\beta$. If $\beta$ is negative, then there are $(m-1) / 2$ roots of negative real part among all possible $m$-th roots of $\beta$. The result of Proposition 4.1 follows.

\subsection{A new formulation of Theorem 3.4 in flat coordinates}

As was already pointed out in [4,7], the construction of a $K$-symmetrizer is tedious in the $z$-space because the variable $z$ belongs to the exterior of the curved unit disk. We are going to reformulate the problem with a matrix depending on a new parameter $\tau$ that belongs to a half-plane.

Lemma 4.2. Under the assumptions of Theorem 3.4, let $\xi \in[0,2 \pi$ [ denote the argument of $\underline{\kappa}$. Then the series

$$
M_{\sharp}(\tau):=i \underline{\xi} I+\sum_{n=1}^{+\infty} \frac{(-1)^{n-1}}{n}\left(\frac{1}{\underline{\kappa}} M\left(\underline{z} \mathrm{e}^{\tau}\right)-I\right)^{n},
$$

defines a holomorphic function $M_{\sharp}$ on a neighborhood $\mathcal{V}_{\sharp}$ of 0 , with values in $\mathcal{M}_{m}(\mathbb{C})$. For all z sufficiently close to $\underline{z}$, we have

$$
M(z)=\exp M_{\sharp}\left(\ln \frac{z}{\underline{z}}\right) .
$$

Moreover, $M_{\sharp}(\tau)$ has no purely imaginary eigenvalue when $\tau \in \mathcal{V}_{\sharp}$ has positive real part, and the lower left coefficient of $M_{\sharp}^{\prime}(0)$ equals $\alpha \underline{z} / \underline{\kappa}$.

Proof of Lemma 4.2. The spectral radius of the matrix $M\left(\underline{z} \mathrm{e}^{\tau}\right) / \underline{\kappa}-I$ is zero when $\tau=0$. Consequently the spectral radius of $M\left(\underline{z} \mathrm{e}^{\tau}\right) / \underline{\kappa}-I$ remains smaller than $1 / 2$ when $\tau$ belongs to a small neighborhood $\mathcal{V}_{\sharp}$ of 0 . Then the series (4.1) converges normally and defines a holomorphic function $M_{\sharp}$ on $\mathcal{V}_{\sharp}$. The matrix $M_{\sharp}$ is a logarithm of $M\left(\underline{z} \mathrm{e}^{\tau}\right)$, see for instance [13, page 60]. When $\tau$ has positive real part, $M_{\sharp}(\tau)$ has no purely imaginary eigenvalue. Otherwise $M\left(\underline{z} \mathrm{e}^{\tau}\right)$ would have an eigenvalue on $\mathbb{S}^{1}$ which is ruled out by the assumptions on $M$. 
It remains to compute the lower left coefficient of $M_{\sharp}^{\prime}(0)$. We make use of the following formula that is fully justified in [13, page 78]:

$$
\begin{aligned}
\operatorname{dexp}_{\left.\right|_{A}} B & :=\left.\frac{\mathrm{d}}{\mathrm{d} \zeta} \exp (A+\zeta B)\right|_{\zeta=0}=\exp (A) \sum_{\nu=0}^{+\infty} \frac{(-1)^{\nu}}{(v+1) !}(\operatorname{ad} A)^{\nu} B, \\
(\operatorname{ad} A) B & :=A B-B A .
\end{aligned}
$$

Let us now differentiate the relation $M\left(\underline{z} \mathrm{e}^{\tau}\right)=\exp M_{\sharp}(\tau)$ with respect to $\tau$, and evaluate at $\tau=0$. We obtain

$$
\underline{z} M^{\prime}(\underline{z})=\operatorname{dexp}_{\left.\right|_{M_{\sharp}(0)}} M_{\sharp}^{\prime}(0) .
$$

Using the relation $M(\underline{z})=\underline{\kappa}(I+N)$, we get

$$
M_{\sharp}(0)=i \underline{\xi} I+N_{\sharp}, \quad N_{\sharp}:=\sum_{k=1}^{m-1} \frac{(-1)^{k-1}}{k} N^{k} .
$$

Using (4.2), (4.3) reads

$$
\underline{z} M^{\prime}(\underline{z})-\underline{\kappa} M_{\sharp}^{\prime}(0)=\underline{\kappa} N M_{\sharp}^{\prime}(0)+\underline{\kappa}(I+N) \sum_{\nu=1}^{+\infty} \frac{(-1)^{\nu}}{(v+1) !}\left(\operatorname{ad} N_{\sharp}\right)^{\nu} M_{\sharp}^{\prime}(0) .
$$

Observe that $N_{\sharp}$ can we written as $N_{\sharp}=N Q(N)=Q(N) N$ where $Q$ is a polynomial. Moreover, for all $B \in \mathcal{M}_{m}(\mathbb{C})$, the last row of $N B$ and the first column of $B N$ are zero. Consequently the lower left coefficient of $\left(\operatorname{ad} N_{\sharp}\right) B$ is zero for all matrix $B$. The relation (4.4) thus shows that the lower left coefficient $\alpha_{\sharp}$ of $M_{\sharp}^{\prime}(0)$ satisfies $\underline{z} \alpha=\underline{\kappa} \alpha_{\sharp}$. The proof of Lemma 4.2 is complete.

The following result is originally due to Ralston.

Lemma 4.3 (Ralston [14]). Let $M_{\sharp}$ be defined by (4.1). Then up to shrinking the neighborhood $\mathcal{V}_{\sharp}$ of 0 on which $M_{\sharp}$ is defined, there exists a holomorphic function $Q$ defined on $\mathcal{V}_{\sharp}$ with values in $G l_{m}(\mathbb{C})$, and there exist holomorphic functions $b_{1}, \ldots, b_{m}$ on $\mathcal{V}_{\sharp}$ such that for all $\tau \in \mathcal{V}_{\sharp}$, there holds

$$
M_{\triangleright}(\tau):=Q(\tau)^{-1} M_{\sharp}(\tau) Q(\tau)=i \underline{\xi} I+i N+\left(\begin{array}{cccc}
b_{1}(\tau) & 0 & \cdots & 0 \\
\vdots & \vdots & & \vdots \\
b_{m}(\tau) & 0 & \cdots & 0
\end{array}\right) .
$$

Moreover, $M_{b}(\tau)$ has no purely imaginary eigenvalue when $\tau$ has positive real part, and the function $b_{m}$ satisfies $b_{m}^{\prime}(0)=(-i)^{m-1} \alpha \underline{z} / \underline{\kappa} \neq 0$. If $m$ is even, then $\operatorname{Im} b_{m}^{\prime}(0) \leq 0$. If $m$ is odd, then $b_{m}^{\prime}(0) \in \mathbb{R}$. 
We refer to [14] and [3, Chapter 7] for the proof of this Lemma. The property of $b_{m}^{\prime}(0)$ follows from Proposition 4.1 .

The number of eigenvalues of $M_{b}(\tau)$ with negative real part does not depend on $\tau$ as long as $\tau$ has positive real part. This number coincides with the number $\mu$ of stable eigenvalues of $M\left(\underline{z} \mathrm{e}^{\tau}\right)$. Rephrasing Proposition 4.1, we have

$$
\mu=\frac{m}{2} \quad \text { if } m \text { is even, } \quad \mu= \begin{cases}\frac{m+1}{2} & \text { if } m \text { is odd and } b_{m}^{\prime}(0)<0, \\ \frac{m-1}{2} & \text { if } m \text { is odd and } b_{m}^{\prime}(0)>0 .\end{cases}
$$

Theorem 3.4 is a consequence of the following analogous result in flat coordinates.

Theorem 4.4. Let $\xi \in \mathbb{R}$, let $m \geq 2$ be an integer. Let $b_{1}, \ldots, b_{m}$ denote some holomorphic functions on some neighborhood $\mathcal{V}_{b}$ of 0 , that vanish at 0 . Let us define a matrix $M_{b}(\tau)$ by the formula

$$
\forall \tau \in \mathcal{V}_{b}, \quad M_{b}(\tau):=i \underline{\xi} I+i N+\left(\begin{array}{cccc}
b_{1}(\tau) & 0 & \cdots & 0 \\
\vdots & \vdots & & \vdots \\
b_{m}(\tau) & 0 & \cdots & 0
\end{array}\right) .
$$

Let us assume that $M_{b}$ satisfies the following conditions:

- for all $\tau \in \mathcal{V}_{b}$ with $\operatorname{Re} \tau>0, \operatorname{sp}\left(M_{b}(\tau)\right) \cap i \mathbb{R}=\emptyset$,

- if $m$ is even, $b_{m}^{\prime}(0) \neq 0$ and $\operatorname{Im} b_{m}^{\prime}(0) \leq 0$,

- if $m$ is odd, $b_{m}^{\prime}(0) \in \mathbb{R} \backslash\{0\}$.

Let the integer $\mu$ be defined by (4.5), and introduce the decomposition

$$
\forall W \in \mathbb{C}^{m}, \quad W=\left(\begin{array}{l}
W^{s} \\
W^{u}
\end{array}\right), \quad W^{s} \in \mathbb{C}^{\mu}, \quad W^{u} \in \mathbb{C}^{m-\mu} .
$$

Then for all $K \geq 1$, there exists a neighborhood $\mathcal{V}_{K}^{b}$ of 0 , there exists a $\mathcal{C}^{\infty}$ function $S_{K}^{b}$ on $\mathcal{V}_{K}^{b}$ with values in $\mathcal{H}_{m}$, and there exists a constant $c_{K}^{b}>0$ such that the following properties hold:

- for all $\tau \in \mathcal{V}_{K}^{b}$ with $\operatorname{Re} \tau \geq 0, \operatorname{Re}\left(S_{K}^{b}(\tau) M_{b}(\tau)\right) \geq c_{K}^{b}(\operatorname{Re} \tau) I$,

- for all $\tau \in \mathcal{V}_{K}^{b}$ and for all $W \in \mathbb{C}^{m}, W^{*} S_{K}^{b}(\tau) W \geq K^{2}\left|W^{u}\right|^{2}-\left|W^{s}\right|^{2}$.

Proof of Theorem 3.4 using Theorem 4.4. Let us now show why the result of Theorem 4.4 implies the result of Theorem 3.4. Using Theorem 4.4, we already know that the matrix $M_{b}$ defined in Lemma 4.3 admits a symmetrizer $S_{K}^{b}$ for all $K \geq 1$. The properties satisfied by $S_{K}^{b}$ are those stated in Theorem 4.4. In the same spirit as Lemma 3.6, one easily shows that the existence of such symmetrizers is invariant under $\mathcal{C}^{\infty}$ changes of basis. More precisely, the matrix $M_{\sharp}$ defined in Lemma 4.2 
equals $M_{b}$ up to a smooth change of basis, see Lemma 4.3. Thus for all $K \geq 1$, there exists a neighborhood $\mathcal{V}_{K}^{\sharp}$ of 0 , there exists a $\mathcal{C}^{\infty}$ function $S_{K}^{\sharp}$ on $\mathcal{V}_{K}^{\sharp}$ with values in $\mathcal{H}_{m}$, and there exists a constant $c_{K}^{\sharp}>0$ such that the following properties hold:

- for all $\tau \in \mathcal{V}_{K}^{\sharp}$ with $\operatorname{Re} \tau>0, \operatorname{Re}\left(S_{K}^{\sharp}(\tau) M_{\sharp}(\tau)\right) \geq c_{K}^{\sharp}(\operatorname{Re} \tau) I$,

- for all $\tau \in \mathcal{V}_{K}^{\sharp}$ and for all $W \in \mathbb{C}^{m}, W^{*} S_{K}^{\sharp}(\tau) W \geq K^{2}\left|W^{u}\right|^{2}-\left|W^{s}\right|^{2}$.

Let $K \geq 1$, and consider the function $S_{K}^{\sharp}$ defined on a neighborhood $\mathcal{V}_{K}^{\sharp}$ of 0 with values in $\mathcal{H}_{m}$. Let $z$ belong to a sufficiently small neighborhood $\mathcal{O}_{K}$ of $\underline{z}$ so that $\ln (z / \underline{z}) \in \mathcal{V}_{K}^{\sharp}$. We define $S_{K}(z):=S_{K}^{\sharp}(\ln (z / \underline{z}))$. We are going to check that $S_{K}$ defines a $K$-symmetrizer for the matrix $M(z)$. We recall that in Lemma 4.2, the matrix $M_{\sharp}$ was constructed in such a way that the relation $M(z)=\exp M_{\sharp}(\ln (z / z))$ holds for $z$ sufficiently close to $\underline{z}$.

Let $z \in \overline{\mathcal{U}} \cap \mathcal{O}_{K}$. Then we know that $S_{K}(z)$ is Hermitian, and for all $W \in \mathbb{C}^{m}$ we have

$$
W^{*} S_{K}(z) W=W^{*} S_{K}^{\sharp}(\ln (z / \underline{z})) W \geq K^{2}\left|W^{u}\right|^{2}-\left|W^{s}\right|^{2} .
$$

Moreover, the calculations of [7, page 685] show that there exists a constant $c_{K}>0$, possibly smaller than the constant $c_{K}^{\sharp}$, such that

$$
M(z)^{*} S_{K}(z) M(z)-S_{K}(z) \geq c_{K} \frac{|z|-1}{|z|} I .
$$

We have thus proved that $S_{K}$ is a $K$-symmetrizer for $M$. The corresponding vector space $\underline{\mathbb{E}}^{s}$ in the decomposition of $\mathbb{C}^{m}$ is the vector space spanned by the $\mu$ first vectors in the canonical basis of $\mathbb{C}^{m}$ :

$$
\underline{\mathbb{E}}^{s}=\left\{\left(\begin{array}{c}
W^{s} \\
0
\end{array}\right), \quad W^{s} \in \mathbb{C}^{\mu}\right\}, \quad \underline{\mathbb{E}}^{u}=\left\{\left(\begin{array}{c}
0 \\
W^{u}
\end{array}\right), \quad W^{u} \in \mathbb{C}^{m-\mu}\right\} .
$$

As claimed in Theorem 3.4, the dimension of $\underline{\mathbb{E}}^{s}$ coincides with the number of stable eigenvalues of $M(z)$ when $|z|>1$.

It remains to prove Theorem 4.4, which is done in the following sections. Let us already observe that the existence of the symmetrizer $S_{K}^{b}$ in Theorem 4.4 does not depend on the real number $\xi$. More precisely, if the symmetrizer $S_{K}^{b}$ works for one value of $\xi$, then it also works for any value of $\xi$. We shall therefore assume from now on that $\xi$ is zero, which simplifies a little bit the notation.

Theorem 4.4 is due to Kreiss [9], see also [2,3,14], in the case where all functions $b_{1}, \ldots, b_{m}$ have purely imaginary values when $\tau$ is purely imaginary. In particular, all derivatives $b_{1}^{\prime}(0), \ldots, b_{m}^{\prime}(0)$ should be real, $b_{1}^{\prime \prime}(0), \ldots, b_{m}^{\prime \prime}(0)$ should be purely imaginary and so on. In our framework, there is no reason why $b_{j}(\tau)$ should have purely imaginary values when $\tau$ is purely imaginary. This phenomenon was 
already highlighted in [4, Lemma 5.2] where we proved Theorem 4.4 in the special case $m=2, \operatorname{Im} b_{2}^{\prime}(0)<0$. We extend here the result of [4] to the general framework of Theorem 4.4. This is done by first classifying the matrices $M_{b}$ according to the dissipation index defined in Proposition 4.5 below. We then construct the symmetrizer $S_{K}^{b}$ in the various possible cases depending on the size $m$ and on the dissipation index.

For simplicity, we omit the index or superscript $b$ from now on.

\subsection{The dissipation index. Classification of all possible cases}

This paragraph is devoted to the following result.

Proposition 4.5. Let $M$ satisfy all the assumptions of Theorem 4.4 with $\xi=0$. Then there exists a unique holomorphic function $\ell$ defined on a neighborhood $\mathcal{W}$ of 0 such that for all $(\tau, \zeta) \in \mathcal{V} \times \mathcal{W}$, there holds

$$
\operatorname{det}(M(\tau)-\zeta I)=\vartheta(\tau, \zeta)(\tau-\ell(\zeta))
$$

where $\vartheta$ is holomorphic and does not vanish on $\mathcal{V} \times \mathcal{W}$. Furthermore, the function $\ell$ satisfies

$$
\ell(0)=\ell^{\prime}(0)=\cdots=\ell^{(m-1)}(0)=0, \quad \ell^{(m)}(0)=\frac{m !}{i^{m-1} b_{m}^{\prime}(0)} \neq 0,
$$

and one of the following two properties holds true:

- $\ell(\zeta) \in i \mathbb{R}$ for all $\zeta \in i \mathbb{R} \cap \mathcal{W}$, or equivalently $i^{\nu-1} \ell^{(\nu)}(0) \in \mathbb{R}$ for all integer $v$,

- there exists a smallest integer $m_{0}$, and this integer $m_{0}$ is even, and there exists a constant $c>0$ such that for all $\xi \in \mathbb{R} \cap \mathcal{W}$, there holds $\operatorname{Re} \ell(i \xi) \leq-c \xi^{m_{0}}$. This condition equivalently reads

$$
\forall v=0, \ldots, m_{0}-1, \quad i^{\nu-1} \ell^{(v)}(0) \in \mathbb{R}, \quad \text { and } \quad(-1)^{m_{0} / 2} \operatorname{Re} \ell^{\left(m_{0}\right)}(0)<0 .
$$

In the first case, we define the dissipation index of $M$ as $+\infty$ while in the second case, we define the dissipation index of $M$ as $m_{0}$. The dissipation index is always larger than or equal to $m$.

Proof of Proposition 4.5. The existence of the holomorphic function $\ell$ follows from the Weierstrass preparation Theorem by simply noting that

$$
\left.\frac{\partial}{\partial \tau} \operatorname{det}(M(\tau)-\zeta I)\right|_{(\tau, \zeta)=(0,0)}=(-i)^{m-1} b_{m}^{\prime}(0) \neq 0 .
$$

Once we know that $\ell$ exists, we evaluate (4.6) at $\tau=0$, and we obtain the relation

$$
(-\zeta)^{m}=\operatorname{det}(M(0)-\zeta I)=-\vartheta(0, \zeta) \ell(\zeta), \quad \vartheta(0,0)=(-i)^{m-1} b_{m}^{\prime}(0) \neq 0
$$

Differentiating $m$ times with respect to $\zeta$ and evaluating at $\zeta=0$, we obtain (4.7). 
We know that for $\tau \in \mathcal{V}$ of positive real part, $M(\tau)$ has no purely imaginary eigenvalue. This implies that for $\zeta \in i \mathbb{R}$ sufficiently small, $\ell(\zeta)$ has nonpositive real part.

Let us consider the real function $\operatorname{Re} \ell(i \xi)$ of the real variable $\xi$, which is defined on an interval ] $-\xi_{0}, \xi_{0}$ [. This function vanishes at 0 and has nonpositive values. There are two possible cases: either all derivatives at 0 vanish (case 1), or there exists a smallest integer $m_{0}$ such that the derivative of order $m_{0}$ is non-zero (case 2).

In case 1 , we have $\ell^{(v)}(0) \in \mathbb{R}$ if $v$ is odd and $\ell^{(v)}(0) \in i \mathbb{R}$ if $v$ is even. Then we can expand $\ell(i \xi)$ in power series for small $\xi$ because $\ell$ is holomorphic, and we find that $\ell(i \xi) \in i \mathbb{R}$ for all small real $\xi$. In case 2 , the integer $m_{0}$ is necessarily even because 0 is a maximum of $\operatorname{Re} \ell(i \xi)$. Then we find that the $m_{0}$-th derivative of $\operatorname{Re} \ell(i \xi)$ at 0 is negative because the function is nonpositive, and the conclusion follows from a Taylor expansion.

The terminology for the dissipation index may be a little confusing. We warn the reader that the lower is the dissipation index, the more dissipative the block will be. The case where the dissipation index is infinite corresponds to a non-dissipative block (in some sense a hyperbolic block). Our terminology is similar to the notion of dissipative scheme for the Cauchy problem, see [6, Definition 5.2.1].

There are now four cases to consider for the proof of Theorem 4.4. The construction of the symmetrizer varies from one case to the other.

- Case I: $m$ is even and the dissipation index $m_{0}$ equals $m$.

- Case II: $m$ is even and the dissipation index $m_{0}$ is larger than $m$ but finite.

- Case III: $m$ is odd and the dissipation index $m_{0}$ is finite.

- Case IV: the dissipation index is infinite ( $m$ is either even or odd).

We are now going to construct a symmetrizer for each case. For the reader's convenience, we present the proofs with an increasing degree of difficulty. This implies some repeated arguments from time to time but we hope that it will clarify the construction. The proof in Section 5 for case I is a generalization of [4, Theorem 2.7] where we dealt with the case $m=m_{0}=2$. It is also important to understand the construction of the symmetrizer in case I in view of the more involved cases II and III. The latter have never been considered in the literature so far.

Case IV is somehow simpler since it can be treated with the standard Kreiss symmetrizers of [9]. In particular, we shall see that in case IV, $M(i \delta)$ has purely imaginary coefficients while in cases I, II and III, $M(i \delta)$ does not have purely imaginary coefficients.

\section{Construction of a symmetrizer: case I}

We recall for clarity that we consider a function $M$ that is holomorphic on a neighborhood $\mathcal{V}$ of 0 with values in $\mathcal{M}_{m}(\mathbb{C}), m \geq 2$, and whose expression is given 
by

$$
\forall \tau \in \mathcal{V}, \quad M(\tau)=i N_{m}+\left(\begin{array}{cccc}
b_{1}(\tau) & 0 & \cdots & 0 \\
\vdots & \vdots & & \vdots \\
b_{m}(\tau) & 0 & \cdots & 0
\end{array}\right), \quad N_{m}=\left(\begin{array}{cccc}
0 & 1 & & 0 \\
\vdots & \ddots & \ddots & \\
\vdots & & \ddots & 1 \\
0 & \cdots & \cdots & 0
\end{array}\right)
$$

All functions $b_{1}, \ldots, b_{m}$ vanish at 0 . Case I corresponds to a function $b_{m}$ that satisfies $\operatorname{Im} b_{m}^{\prime}(0)<0$, see Proposition 4.5. In particular, $b_{m}(i \delta)$ is not purely imaginary for small real $\delta$. A numerical scheme that produces a block of case I with arbitrarily large $m$ is constructed in Section 9. We also recall that we have $m=2 \mu$, see (4.5), and that any vector $W \in \mathbb{C}^{m}$ is decomposed as $W=\left(W^{s}, W^{u}\right)$ where $W^{s} \in \mathbb{C}^{\mu}$ is the vector formed by the $\mu$ first coordinates of $W$, while $W^{u} \in \mathbb{C}^{\mu}$ is the vector formed by the $\mu$ last coordinates of $W$.

In the analysis of all cases I-IV, the construction of the symmetrizer $S_{K}(\tau)$ is based on the following observation. Writing $\tau=\gamma+i \delta$, we first expand $M(\tau)$ as

$$
M(\tau)=M(i \delta)+\gamma M^{\prime}(0)+\gamma r(\tau)
$$

where $r$ is continuous with respect to $\tau \in \mathcal{V}$ and $r(0)=0$. Then we choose the symmetrizer $S_{K}(\tau)$ under the form

$$
S_{K}(\tau)=\mathbf{S}(\delta)+\gamma \mathbf{H}
$$

where $\mathbf{S}(\delta)$ is Hermitian and $\mathbf{H}$ is a constant Hermitian matrix. The following Lemma is based on the above expansion of $M(\tau)$. Its elementary proof is omitted.

Lemma 5.1. Assume that for all $K \geq 1$, we can construct a $\mathcal{C}^{\infty}$ function $\mathbf{S}$ on some interval $]-\delta_{K}, \delta_{K}$ [ with values in $\mathcal{H}_{m}$, and a matrix $\mathbf{H} \in \mathcal{H}_{m}$ such that the following properties hold:

i) for all $\delta \in]-\delta_{K}, \delta_{K}[, \operatorname{Re}(\mathbf{S}(\delta) M(i \delta)) \geq 0$,

ii) for all $W \in \mathbb{C}^{m}, W^{*} \mathbf{S}(0) W \geq\left(K^{2}+1 / 2\right)\left|W^{u}\right|^{2}-\left|W^{s}\right|^{2} / 2$,

iii) $\operatorname{Re}\left(\mathbf{S}(0) M^{\prime}(0)+i \mathbf{H} N_{m}\right)$ is positive definite.

Then the result of Theorem 4.4 holds with the symmetrizer $S_{K}$ in (5.2).

The construction of $\mathbf{S}$ varies from one case to the other, because the behavior of the functions $b_{j}$ when $\tau$ is purely imaginary is encoded in the dissipation index $m_{0}$. In particular, the choice in [9] of a real symmetric matrix $\mathbf{S}(\delta)$ such that $\mathbf{S}(\delta) M(i \delta)$ is skew-Hermitian is not convenient in cases I, II and III (see Appendix B). However, we shall see in the analysis of cases II and III that the larger the dissipation index $m_{0}$ is, the more our construction mimics Kreiss' choice. 
Let $K \geq 1$, and let us construct a matrix $\mathbf{S}(\delta)$ satisfying conditions i) and ii) of Lemma 5.1 in case I. We decompose the matrix $M(i \delta)$ as follows:

$$
M(i \delta)=i\left(\begin{array}{cc}
N_{\mu} & \tilde{N}_{\mu} \\
0 & N_{\mu}
\end{array}\right)+\left(\begin{array}{cc}
\delta \beta_{1}(\delta) & 0 \\
i \delta B_{1}+\delta^{2} \beta_{2}(\delta) & 0
\end{array}\right),
$$

where each block in (5.3) represents a matrix of size $\mu=m / 2$. The coefficients of the matrix $\widetilde{N}_{\mu}$ vanish, except the lower left coefficient that equals 1 . When $m$ equals 2 , we use the convention $N_{1}=0, \widetilde{N}_{1}=1$. Moreover, $B_{1}$ is a constant matrix defined by

$$
B_{1}:=\left(\begin{array}{cccc}
b_{\mu+1}^{\prime}(0) & 0 & \cdots & 0 \\
\vdots & \vdots & & \vdots \\
b_{m}^{\prime}(0) & 0 & \cdots & 0
\end{array}\right) \in \mathcal{M}_{\mu}(\mathbb{C}) .
$$

The matrices $\beta_{1}(\delta), \beta_{2}(\delta) \in \mathcal{M}_{\mu}(\mathbb{C})$ depend analytically on $\delta$ but we have no information about their coefficients. In case I, the only piece of information we have is on the lower left coefficient of $B_{1}: \operatorname{Im} b_{m}^{\prime}(0)<0$.

We choose the symmetrizer $\mathbf{S}(\delta)$ under the following form ${ }^{3}$ :

$$
\mathbf{S}(\delta):=\left(\begin{array}{cc}
\delta^{2} \mathbf{A}_{2} & \mathbf{C}_{0}+\delta \mathbf{C}_{1} \\
\mathbf{C}_{0}^{*}+\delta \mathbf{C}_{1}^{*} & \mathbf{D}_{0}
\end{array}\right),
$$

where $\mathbf{A}_{2}, \mathbf{D}_{0}$ belong to $\mathcal{H}_{\mu}$, and $\mathbf{C}_{0}, \mathbf{C}_{1}$ belong to $\mathcal{M}_{\mu}(\mathbb{C})$. We shall first fix $\mathbf{C}_{0}$, then $\mathbf{D}_{0}$, then $\mathbf{C}_{1}$ and eventually $\mathbf{A}_{2}$. Computing the product of $\mathbf{S}(\delta)$ in (5.5) with $M(i \delta)$ in (5.3) and taking the real part, we obtain

$$
\begin{aligned}
& \operatorname{Re}(\mathbf{S}(\delta) M(i \delta))= \\
& \left(\begin{array}{c}
\delta \operatorname{Re}\left(i \mathbf{C}_{0} B_{1}\right)+\delta^{2} \operatorname{Re}\left(i \mathbf{A}_{2} N_{\mu}+i \mathbf{C}_{1} B_{1}+\mathbf{C}_{0} \beta_{2}(0)\right) \\
i \\
\frac{i}{2}\left(\mathbf{C}_{0}^{*} N_{\mu}-N_{\mu}^{*} \mathbf{C}_{0}^{*}\right)+\frac{i \delta}{2}\left(\mathbf{C}_{1}^{*} N_{\mu}-N_{\mu}^{*} \mathbf{C}_{1}^{*}+\mathbf{D}_{0} B_{1}-i \mathbf{C}_{0}^{*} \beta_{1}(0)\right) \\
\operatorname{Re}\left(i \mathbf{D}_{0} N_{\mu}+i \mathbf{C}_{0}^{*} \widetilde{N}_{\mu}\right)
\end{array}\right) \\
& +\left(\begin{array}{cc}
O\left(\delta^{3}\right) & \star \\
O\left(\delta^{2}\right) & O(\delta)
\end{array}\right), \quad \text { (5.6) }
\end{aligned}
$$

where $\star$ denotes here and from now the only possible matrix such that the whole $m \times m$ matrix is Hermitian. As in [9], see also [3, Chapter VII.5], we shall use repeatedly the following fact:

Lemma 5.2. Let $v \geq 1$ be an integer. A matrix $S \in \mathcal{M}_{v}(\mathbb{C})$ satisfies $S N_{v}=N_{v}^{*} S$ if and only if $S$ has the form

$$
S=\left(\begin{array}{cccc}
0 & \cdots & 0 & s_{1} \\
\vdots & 0 & s_{1} & s_{2} \\
0 & s_{1} & s_{2} & \vdots \\
s_{1} & s_{2} & \cdots & s_{v}
\end{array}\right) .
$$

3 The reader will observe that this choice is similar to the form we had used in [4], and is completely different from the choice in [9]. 
In particular, $S \in \mathcal{H}_{v}$ satisfies $\operatorname{Re}\left(i S N_{v}\right)=0$ if and only if $S$ is a real symmetric matrix of the form (5.7).

We first fix the matrix $\mathbf{C}_{0}$.

Lemma 5.3. Let the numbers $\rho_{2}, \ldots, \rho_{\mu} \in \mathbb{C}$ be determined as the solution to the triangular system

$$
\left(\begin{array}{ccc}
b_{m}^{\prime}(0) & & 0 \\
\vdots & \ddots & \\
b_{\mu+2}^{\prime}(0) & \cdots & b_{m}^{\prime}(0)
\end{array}\right)\left(\begin{array}{c}
\rho_{2} \\
\vdots \\
\rho_{\mu}
\end{array}\right)=-\overline{b_{m}^{\prime}(0)}\left(\begin{array}{c}
b_{m-1}^{\prime}(0) \\
\vdots \\
b_{\mu+1}^{\prime}(0)
\end{array}\right)
$$

Let the matrix $\mathbf{C}_{0} \in \mathcal{M}_{\mu}(\mathbb{C})$ be defined by

$$
\mathbf{C}_{0}:=\left(\begin{array}{cccc}
0 & \cdots & 0 & \overline{b_{m}^{\prime}(0)} \\
\vdots & 0 & \overline{b_{m}^{\prime}(0)} & \rho_{2} \\
\frac{0}{b_{m}^{\prime}(0)} & \overline{b_{m}^{\prime}(0)} & \rho_{2} & \vdots \\
\rho_{2} & \cdots & \rho_{\mu}
\end{array}\right) .
$$

Then the matrix $\mathbf{C}_{0}$ satisfies $\operatorname{Re}\left(i \mathbf{C}_{0} B_{1}\right)=0, \mathbf{C}_{0}^{*} N_{\mu}=N_{\mu}^{*} \mathbf{C}_{0}^{*}$, and the upper left coefficient of $\operatorname{Re}\left(i \mathbf{C}_{0}^{*} \tilde{N}_{\mu}\right)$ is positive.

The proof of Lemma 5.3 follows from straightforward algebraic manipulations, from Lemma 5.2, and from the fact that $b_{m}^{\prime}(0)$ has negative imaginary part. The details are left to the reader. In view of our choice (5.5) and of the relation (5.6), we now wish to construct a matrix $\mathbf{D}_{0} \in \mathcal{H}_{\mu}$ that is positive definite - in order to satisfy condition ii) in Lemma 5.1 - and such that the real part of $i\left(\mathbf{D}_{0} N_{\mu}+\mathbf{C}_{0}^{*} \widetilde{N}_{\mu}\right)$ is positive definite. The construction of $\mathbf{D}_{0}$ is based on the following general result that is proved in Appendix A.

Lemma 5.4. Let $C_{1}, C_{2} \in \mathbb{R}$, let $c>0$, and let $v \geq 1$ be an integer. Then there exists a matrix $H \in \mathcal{H}_{v}$ such that for all $W=\left(W_{1}, \ldots, W_{v}\right) \in \mathbb{C}^{v}$, the following inequalities hold true

$$
\begin{aligned}
& W^{*} H W \geq C_{1}|W|^{2}, \\
& W^{*} \operatorname{Re}\left(i H N_{v}\right) W \geq-c\left|W_{1}\right|^{2}+C_{2} \sum_{j=2}^{v}\left|W_{j}\right|^{2} .
\end{aligned}
$$

Corollary 5.5. Let $v \geq 1$ be an integer and let $H_{1} \in \mathcal{H}_{v}$. If the upper left coefficient of $H_{1}$ is positive, then there exists $H_{2} \in \mathcal{H}_{v}$ such that $\operatorname{Re}\left(i H_{2} N_{v}\right)+H_{1}$ is positive definite. 
Since the upper left coefficient of $\operatorname{Re}\left(i \mathbf{C}_{0}^{*} \widetilde{N}_{\mu}\right)$ is positive (see Lemma 5.3), Young's inequality shows that there exists a constant $\tilde{c}>0$ such that

$$
\forall W \in \mathbb{C}^{\mu}, \quad W^{*} \operatorname{Re}\left(i \mathbf{C}_{0}^{*} \tilde{N}_{\mu}\right) W \geq 2 \tilde{c}\left|W_{1}\right|^{2}-\frac{1}{\tilde{c}} \sum_{j=2}^{\mu}\left|W_{j}\right|^{2} .
$$

We apply Lemma 5.4 with $C_{1}:=K^{2}+1 / 2+2\left\|\mathbf{C}_{0}\right\|^{2}, C_{2}:=\tilde{c}+1 / \tilde{c}$, and $c:=\tilde{c}$. We obtain that there exists $\mathbf{D}_{0} \in \mathcal{H}_{\mu}$ such that

$$
\begin{aligned}
\forall W \in \mathbb{C}^{\mu}, \quad W^{*} \mathbf{D}_{0} W \geq\left(K^{2}+\frac{1}{2}+2\left\|\mathbf{C}_{0}\right\|^{2}\right)|W|^{2}, \\
W^{*} \operatorname{Re}\left(i \mathbf{D}_{0} N_{\mu}\right) W \geq-\tilde{c}\left|W_{1}\right|^{2}+\left(\tilde{c}+\frac{1}{\tilde{c}}\right) \sum_{j=2}^{\mu}\left|W_{j}\right|^{2} .
\end{aligned}
$$

If we combine (5.9) and (5.11), we already see that the lower right block in the right-hand side of (5.6) satisfies

$$
\operatorname{Re}\left(i \mathbf{D}_{0} N_{\mu}+i \mathbf{C}_{0}^{*} \tilde{N}_{\mu}\right) \geq \tilde{c} I .
$$

Moreover, (5.10) shows that we have the following inequality for all $W \in \mathbb{C}^{m}$ :

$W^{*} \mathbf{S}(0) W=\left(W^{u}\right)^{*} \mathbf{D}_{0} W^{u}+2 \operatorname{Re}\left(\left(W^{s}\right)^{*} \mathbf{C}_{0} W^{u}\right) \geq\left(K^{2}+\frac{1}{2}\right)\left|W^{u}\right|^{2}-\frac{1}{2}\left|W^{s}\right|^{2}$.

Condition ii) of Lemma 5.1 is thus satisfied. Our choice of $\mathbf{C}_{0}$ and of $\mathbf{D}_{0}$ yields some simplification in (5.6):

$$
\begin{aligned}
\operatorname{Re}(\mathbf{S}(\delta) M(i \delta)) \geq & \left(\begin{array}{cc}
\delta^{2} \operatorname{Re}\left(i \mathbf{A}_{2} N_{\mu}+i \mathbf{C}_{1} B_{1}+\mathbf{C}_{0} \beta_{2}(0)\right) & \star \\
\frac{i \delta}{2}\left(\mathbf{C}_{1}^{*} N_{\mu}-N_{\mu}^{*} \mathbf{C}_{1}^{*}+\mathbf{D}_{0} B_{1}-i \mathbf{C}_{0}^{*} \beta_{1}(0)\right) \tilde{c} I
\end{array}\right) \\
& +\left(\begin{array}{c}
O\left(\delta^{3}\right) \star \\
O\left(\delta^{2}\right)
\end{array}\right)
\end{aligned}
$$

It remains to fix the matrices $\mathbf{C}_{1}$ and $\mathbf{A}_{2}$ such that condition i) in Lemma 5.1 holds. Let us first of all choose $\mathbf{C}_{1}$ of the form given in Lemma 5.2, so that $\mathbf{C}_{1}$ satisfies $\mathbf{C}_{1}^{*} N_{\mu}=N_{\mu}^{*} \mathbf{C}_{1}^{*}$. Applying Young's inequality in (5.12) shows that there exists a constant $\tilde{C}>0$, that does not depend on $\mathbf{C}_{1}$ nor on $\mathbf{A}_{2}$, such that

$$
\begin{aligned}
& \operatorname{Re}(\mathbf{S}(\delta) M(i \delta)) \geq\left(\begin{array}{cc}
\delta^{2}\left(\operatorname{Re}\left(i \mathbf{A}_{2} N_{\mu}+i \mathbf{C}_{1} B_{1}\right)-\tilde{C} I\right) & 0 \\
0 & \frac{\tilde{c}}{2} I
\end{array}\right) \\
& +\left(\begin{array}{cc}
O\left(\delta^{3}\right) & \star \\
O\left(\delta^{2}\right) & O(\delta)
\end{array}\right) .
\end{aligned}
$$

Let us now fix the matrices $\mathbf{C}_{1}$ and $\mathbf{A}_{2}$. 
Lemma 5.6. For all $C_{3} \in \mathbb{R}$, there exists a matrix $E \in \mathcal{M}_{\mu}(\mathbb{C})$ and there exists a matrix $H \in \mathcal{H}_{\mu}$ that satisfy

$$
E N_{\mu}=N_{\mu}^{*} E, \quad \operatorname{Re}\left(i H N_{\mu}+i E B_{1}\right) \geq C_{3} I .
$$

Proof of Lemma 5.6. We first choose the matrix $E \in \mathcal{M}_{\mu}(\mathbb{R})$ of the form

$$
E=\left(\begin{array}{cc}
0 & e \\
& e \\
e & 0
\end{array}\right), \quad e \in \mathbb{R},
$$

so that $E N_{\mu}=N_{\mu}^{*} E$. Then we compute the upper left coefficient of $\operatorname{Re}\left(i E B_{1}\right)$. This coefficient equals $e\left|\operatorname{Im} b_{m}^{\prime}(0)\right|$. We thus fix $e \in \mathbb{R}$ such that the upper left coefficient of $\operatorname{Re}\left(i E B_{1}\right)$ equals $C_{3}+1$. Then there exists a constant $C_{4}>0$ such that for all $W \in \mathbb{C}^{\mu}$ there holds

$$
W^{*} \operatorname{Re}\left(\text { i } E B_{1}\right) W \geq\left(C_{3}+\frac{1}{2}\right)\left|W_{1}\right|^{2}-C_{4} \sum_{j=2}^{\mu}\left|W_{j}\right|^{2} .
$$

It remains to apply Lemma 5.4 above with $c:=1 / 2, C_{2}:=C_{3}+C_{4}$ and with an arbitrary $C_{1}$ (take for instance $C_{1}=0$ ) to construct the Hermitian matrix $H$.

Applying Lemma 5.6 in (5.13), we can pick a matrix $\mathbf{C}_{1} \in \mathcal{M}_{\mu}(\mathbb{C})$ that satisfies $\mathbf{C}_{1}^{*} N_{\mu}=N_{\mu}^{*} \mathbf{C}_{1}^{*}$, and a matrix $\mathbf{A}_{2} \in \mathcal{H}_{\mu}$ such that

$$
\operatorname{Re}\left(i \mathbf{A}_{2} N_{\mu}+i \mathbf{C}_{1} B_{1}\right) \geq\left(\tilde{C}+\frac{\tilde{c}}{2}\right) I .
$$

Consequently, (5.13) reduces to

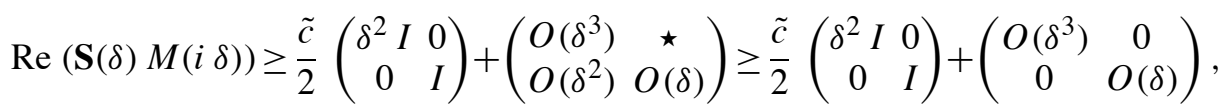

where we have used Young's inequality in the end. Choosing $\delta$ small enough, we have thus constructed a matrix $\mathbf{S}(\delta)$ of the form (5.5) and that satisfies conditions i) and ii) of Lemma 5.1. Moreover, the matrix $\mathbf{C}_{0}$ in the decomposition (5.5) is defined by (5.8).

We now construct the Hermitian matrix $\mathbf{H}$ such that condition iii) in Lemma 5.1 is satisfied. Using the expression (5.8) of $\mathbf{C}_{0}$, the upper left coefficient of the matrix $\operatorname{Re}\left(\mathbf{S}(0) M^{\prime}(0)\right)$ equals $\left|b_{m}^{\prime}(0)\right|^{2}>0$. Corollary 5.5 shows that there exists a matrix $\mathbf{H} \in \mathcal{H}_{m}$ such that $\operatorname{Re}\left(\mathbf{S}(0) M^{\prime}(0)+i \mathbf{H} N_{m}\right)$ is positive definite. Condition iii) in Lemma 5.1 is thus satisfied, which shows that Theorem 4.4 holds in case I.

The analysis of cases II and III below follows the same strategy as for case I. The most difficult part is to guess the form and construct the Hermitian matrix $\mathbf{S}(\delta)$. The construction of $\mathbf{H} \in \mathcal{H}_{m}$ always follows from Corollary 5.5. For clarity, we rephrase and simplify Lemma 5.1 in order to take the result of Corollary 5.5 into account. 
Lemma 5.7. Assume that for all $K \geq 1$, we can construct a $\mathcal{C}^{\infty}$ function $\mathbf{S}$ on some interval ] $-\delta_{K}, \delta_{K}$ [ with values in $\mathcal{H}_{m}$ such that the following properties hold:

i) for all $\delta \in]-\delta_{K}, \delta_{K}[, \operatorname{Re}(\mathbf{S}(\delta) M(i \delta)) \geq 0$,

ii) for all $W \in \mathbb{C}^{m}, W^{*} \mathbf{S}(0) W \geq\left(K^{2}+1 / 2\right)\left|W^{u}\right|^{2}-\left|W^{s}\right|^{2} / 2$,

iii) the upper left coefficient of $\operatorname{Re}\left(\mathbf{S}(0) M^{\prime}(0)\right)$ is positive.

Then the result of Theorem 4.4 holds with a symmetrizer $S_{K}$ of the form (5.2).

\section{Construction of a symmetrizer: case II}

In this section, we consider a matrix $M(\tau)$ of the form (5.1) where $m$ is an even number, and where the dissipation index $m_{0}$ defined in Proposition 4.5 is larger than $m$. The dissipation index gives some information on the holomorphic function $\ell$. First of all, we are going to convert this information on $\ell$ into some information on the derivatives $b_{j}^{(q)}(0)$. This is done in Proposition 6.1 below. According to the values of $m_{0}$ with respect to $m$, we shall construct a symmetrizer. Observe that since both $m$ and $m_{0}$ are even numbers, the Euclidean division of $m_{0}$ by $m$ reads

$$
m_{0}=q_{0} m+2 \mu_{0}, \quad q_{0} \geq 1, \quad 0 \leq \mu_{0} \leq \mu-1 .
$$

\subsection{What does the dissipation condition mean?}

The following proposition gives some information on the derivatives $b_{j}^{(q)}(0)$.

Proposition 6.1. Let $M$ satisfy the assumptions of Theorem 4.4, where without loss of generality $\xi=0$, and correspond to case II ( $m_{0}>m, m$ is even). Let the dissipation index $m_{0}$ satisfy (6.1). Then the following properties hold:

- $b_{m}^{\prime}(0) \in \mathbb{R} \backslash\{0\}$,

- for all $q=1, \ldots, q_{0}-1$ and for all $j=1, \ldots, m, i^{q-1} b_{j}^{(q)}(0) \in \mathbb{R}$,

- for all $j=0, \ldots, 2 \mu_{0}-1, i^{q_{0}-1} b_{m-j}^{\left(q_{0}\right)}(0) \in \mathbb{R}$,

- if $q_{0}$ is even, $b_{m}^{\prime}(0) \operatorname{Im}\left(i^{q_{0}-1} b_{m-2 \mu_{0}}^{\left(q_{0}\right)}(0)\right)<0$,

- if $q_{0}$ is odd, $\operatorname{Im}\left(i^{q_{0}-1} b_{m-2 \mu_{0}}^{\left(q_{0}\right)}(0)\right)<0$.

Proposition 6.1 shows in particular that $b_{m-2} \mu_{0}(i \delta)$ is not a purely imaginary number for all small real $\delta$. Hence $M(i \delta)$ does not have purely imaginary coefficients for small real $\delta$ in case II. The same property holds in case III, see Proposition 7.1 below. 
Proof of Proposition 6.1. We recall that there exists a unique holomorphic function $\ell$ satisfying (4.6). In case II, this function satisfies (4.7) and

$$
\begin{array}{ll} 
& i^{m-1} \ell^{(m)}(0) \in \mathbb{R} \backslash\{0\}, \\
\forall v=m+1, \ldots, m_{0}-1, \quad i^{\nu-1} \ell^{(v)}(0) \in \mathbb{R}, \\
(-1)^{m_{0} / 2} \operatorname{Re} \ell^{\left(m_{0}\right)}(0)<0 .
\end{array}
$$

If we use the form (5.1) of the matrix $M(\tau)$ together with the relation (4.6), we get

$$
0=\operatorname{det}(M(\ell(\zeta))-\zeta I)=(-1)^{m}\left\{\zeta^{m}-\sum_{j=1}^{m} i^{j-1} b_{j}(\ell(\zeta)) \zeta^{m-j}\right\}
$$

Defining the functions

$$
\tilde{\ell}(\omega):=\frac{1}{i} \ell(i \omega), \quad \widetilde{b}_{j}(\theta):=\frac{1}{i} b_{j}(i \theta),
$$

we obtain the relation

$$
\omega^{m}=\sum_{j=1}^{m} \tilde{b}_{j}(\tilde{\ell}(\omega)) \omega^{m-j}
$$

The latter equality holds for all $\omega \in \mathbb{C}$ sufficiently close to 0 . We recall that the functions $\widetilde{b}_{1}, \ldots, \widetilde{b}_{m}, \widetilde{\ell}$ are holomorphic on a neighborhood of 0 .

The proof of Proposition 6.1 then consists in expanding the functions $\tilde{b}_{1}, \ldots$, $\widetilde{b}_{m}, \tilde{\ell}$ near 0 , and in identifying the powers of $\omega$. Let us perform this argument in detail. First of all we assume $q_{0}=1$, and therefore $\mu_{0}>0$. Using (4.7), we have the Taylor expansions

$$
\begin{aligned}
\tilde{\ell}(\omega) & =\frac{\tilde{\ell}^{(m)}(0)}{m !} \omega^{m}+\cdots+\frac{\tilde{\ell}^{(2 m-1)}(0)}{(2 m-1) !} \omega^{2 m-1}+O\left(\omega^{2 m}\right), \\
\tilde{b}_{j}(\tilde{\ell}(\omega)) & =\tilde{b}_{j}^{\prime}(0) \sum_{\nu=m}^{2 m-1} \frac{\tilde{\ell}^{(\nu)}(0)}{\nu !} \omega^{\nu}+O\left(\omega^{2 m}\right) .
\end{aligned}
$$

We use the latter expansion in (6.4) and obtain

$$
\omega^{m}=\sum_{j=1}^{m} \widetilde{b}_{j}^{\prime}(0) \omega^{m-j} \sum_{\nu=m}^{2 m-1} \frac{\tilde{\ell}^{(\nu)}(0)}{\nu !} \omega^{\nu}+O\left(\omega^{2 m}\right) .
$$


Identifying the powers $\omega^{m}, \omega^{m+1}, \ldots, \omega^{m+2 \mu_{0}}$, we obtain

$$
\begin{array}{r}
\widetilde{b}_{m}^{\prime}(0) \frac{\tilde{\ell}^{(m)}(0)}{m !}=1, \\
\widetilde{b}_{m}^{\prime}(0) \frac{\widetilde{\ell}^{(m+1)}(0)}{(m+1) !}+\widetilde{b}_{m-1}^{\prime}(0) \frac{\tilde{\ell}^{(m)}(0)}{m !}=0, \\
\vdots \\
\widetilde{b}_{m}^{\prime}(0) \frac{\tilde{\ell}^{\left(m+2 \mu_{0}\right)}(0)}{\left(m+2 \mu_{0}\right) !}+\cdots+\widetilde{b}_{m-2 \mu_{0}}^{\prime}(0) \frac{\tilde{\ell}^{(m)}(0)}{m !}=0 .
\end{array}
$$

The definition (6.3) gives the relation $\tilde{\ell}^{(v)}(0)=i^{\nu-1} \ell^{(v)}(0)$. Using the properties (6.2), we obtain inductively

$$
\widetilde{b}_{m}^{\prime}(0) \in \mathbb{R} \backslash\{0\}, \quad \widetilde{b}_{m-1}^{\prime}(0), \ldots, \widetilde{b}_{m-2 \mu_{0}+1}^{\prime}(0) \in \mathbb{R} .
$$

Then we multiply (6.6) by the real number $\widetilde{b}_{m}^{\prime}(0)$, take the imaginary part, use $(6.5)$ and (6.2). We find

$$
\operatorname{Im} \widetilde{b}_{m-2 \mu_{0}}^{\prime}(0)<0 \text {. }
$$

We then obtain the result of Proposition 6.1 by using the relations $\widetilde{b}_{j}^{\prime}(0)=b_{j}^{\prime}(0)$, $j=1, \ldots, m$.

Let us now consider the case $q_{0} \geq 2$. We follow the same strategy as above and use the following expansions in (6.4):

$$
\begin{aligned}
\tilde{\ell}(\omega) & =\sum_{\nu=m}^{\left(q_{0}+1\right) m-1} \frac{\tilde{\ell}^{(\nu)}(0)}{\nu !} \omega^{\nu}+O\left(\omega^{\left(q_{0}+1\right) m}\right), \\
\tilde{b}_{j}(\theta) & =\sum_{q=1}^{q_{0}} \frac{\tilde{b}_{j}^{(q)}(0)}{q !} \theta^{q}+O\left(\theta^{q_{0}+1}\right) .
\end{aligned}
$$

We obtain the relation

$$
\omega^{m}=\sum_{j=1}^{m} \omega^{m-j} \sum_{q=1}^{q_{0}} \frac{\tilde{b}_{j}^{(q)}(0)}{q !}\left(\sum_{\nu=m}^{\left(q_{0}+1\right) m-1} \frac{\tilde{\ell}^{(\nu)}(0)}{\nu !} \omega^{\nu}\right)^{q}+O\left(\omega^{\left(q_{0}+1\right) m}\right) .
$$

We first identify the terms $\omega^{m}, \ldots, \omega^{2 m-1}$ on either side of (6.7). Following the same argument as in the case $q_{0}=1$ yields

$$
\widetilde{b}_{m}^{\prime}(0) \in \mathbb{R} \backslash\{0\}, \quad \widetilde{b}_{m-1}^{\prime}(0), \ldots, \widetilde{b}_{1}^{\prime}(0) \in \mathbb{R} .
$$

Then we identify the terms $\omega^{2 m}, \omega^{2 m+1}$ and so on, up to $\omega^{q_{0} m+2 \mu_{0}-1}$. We obtain

$$
\begin{aligned}
& \widetilde{b}_{m}^{\prime \prime}(0), \ldots, \widetilde{b}_{1}^{\prime \prime}(0) \in \mathbb{R}, \quad \ldots, \quad \tilde{b}_{m}^{\left(q_{0}-1\right)}(0), \ldots, \tilde{b}_{1}^{\left(q_{0}-1\right)}(0) \in \mathbb{R}, \\
& \widetilde{b}_{m}^{\left(q_{0}\right)}(0), \ldots, \widetilde{b}_{m-2 \mu_{0}+1}^{\left(q_{0}\right)}(0) \in \mathbb{R} .
\end{aligned}
$$


Let us now identify the term $\omega^{q_{0} m+2 \mu_{0}}=\omega^{m_{0}}$ on either side of (6.7) and get

$$
\begin{aligned}
& 0=\sum_{j=1}^{m} \frac{\tilde{b}_{j}^{\prime}(0)}{1 !} \frac{\tilde{\ell}^{\left(\left(q_{0}-1\right) m+2 \mu_{0}+j\right)}(0)}{\left(\left(q_{0}-1\right) m+2 \mu_{0}+j\right) !} \\
& +\sum_{j=1}^{m} \sum_{q=2}^{q_{0}-1} \frac{\tilde{b}_{j}^{(q)}(0)}{q !} \sum_{\substack{v_{1}, \ldots, v_{q}=m \\
v_{1}+\cdots+v_{q}=\left(q_{0}-1\right) m+2}}^{\left(q_{0}+1\right) m-1} \frac{\tilde{\ell}^{\left(v_{1}\right)}(0)}{v_{1} !} \ldots \frac{\tilde{\ell}^{\left(v_{q}\right)}(0)}{v_{q} !} \\
& +\sum_{j=m-2}^{m} \frac{\tilde{b}_{j}^{\left(q_{0}\right)}(0)}{q_{0} !} \sum_{\substack{v_{1}, \ldots, v_{q_{0}}=m \\
v_{1}+\cdots+v_{q_{0}}=\left(q_{0}-1\right) m+2 \mu_{0}+j}}^{\left(q_{0}+1\right) m-1} \frac{\tilde{\ell}^{\left(v_{1}\right)}(0)}{v_{1} !} \ldots \frac{\tilde{\ell}^{\left(v_{q_{0}}\right)}(0)}{v_{q_{0}} !} .
\end{aligned}
$$

Taking the imaginary part of either side gives

$$
0=\widetilde{b}_{m}^{\prime}(0) \frac{\operatorname{Im} \tilde{\ell}^{\left(m_{0}\right)}(0)}{m_{0} !}+\frac{\operatorname{Im} \tilde{b}_{m-2 \mu_{0}}^{\left(q_{0}\right)}(0)}{q_{0} !}\left(\frac{\tilde{\ell}^{(m)}(0)}{m !}\right)^{q_{0}} .
$$

The conclusion for $\widetilde{b}_{m-2 \mu_{0}}^{\left(q_{0}\right)}(0)$ immediately follows (use (6.5) when $q_{0}$ is odd). We have thus proved all the relations stated in Proposition 6.1.

Our aim is to construct a symmetrizer $\mathbf{S}(\delta) \in \mathcal{H}_{m}$ that satisfies the properties i), ii), iii) of Lemma 5.7. It turns out that the construction of $\mathbf{S}(\delta)$ depends on the integers $q_{0}, \mu_{0}$ in the Euclidean division (6.1). More precisely we shall distinguish the following four possible cases, which correspond to an increasing level of difficulty.

- Case IIa: $\mu_{0}=0, q_{0}$ is even.

- Case IIb: $\mu_{0}=0, q_{0}$ is odd. (This implies $q_{0} \geq 3$.)

- Case IIc: $1 \leq \mu_{0} \leq \mu-1, q_{0}$ is even. (This implies $m \geq 4$.)

- Case IId: $1 \leq \mu_{0} \leq \mu-1, q_{0}$ is odd. (Same remark as for case IIc.)

\subsection{Case IIa}

We shall repeatedly use the following result. We recall that $\mathcal{S}_{v}$ denotes the vector space of real symmetric matrices of size $v$.

Lemma 6.2. Let $v \geq 1$ be an integer, and let $\Psi$ be the linear mapping defined by

$$
\begin{aligned}
\Psi: \mathcal{S}_{v} & \longrightarrow \mathcal{H}_{v} \\
S & \longmapsto \operatorname{Re}\left(i S N_{v}\right) .
\end{aligned}
$$

The kernel of $\Psi$ is made of all matrices $S \in \mathcal{S}_{v}$ of the form (5.7), and the image of $\Psi$ is $\mathcal{H}_{v} \cap i \mathcal{M}_{v}(\mathbb{R})$. 
Proof of Lemma 6.2. The dimension of $\mathcal{S}_{v}$ is $v(v+1) / 2$. Moreover, Lemma 5.2 shows that the kernel of $\Psi$ is made of all matrices $S \in \mathcal{S}_{v}$ of the form (5.7). Thus the kernel of $\Psi$ has dimension $v$, and the image of $\Psi$ has dimension $v(v-1) / 2$.

If $S \in \mathcal{S}_{\nu}$, then $\operatorname{Re}\left(i S N_{v}\right.$ ) is a Hermitian matrix with purely imaginary coefficients. Consequently, the image of $\Psi$ is included in $\mathcal{H}_{v} \cap i \mathcal{M}_{v}(\mathbb{R})$. Moreover, the dimension of $\mathcal{H}_{v} \cap i \mathcal{M}_{v}(\mathbb{R})$ is $v(v-1) / 2$ so the claim of Lemma 6.2 holds.

We decompose the matrix $M(i \delta)$ as follows:

$$
M(i \delta)=i N_{m}+i \sum_{q=1}^{q_{0}} \delta^{q} B_{q}+O\left(\delta^{q_{0}+1}\right)
$$

where the matrices $B_{1}, \ldots, B_{q_{0}}$ are given by ${ }^{4}$

$$
\forall q=1, \ldots, q_{0}, \quad B_{q}:=\frac{i^{q-1}}{q !}\left(\begin{array}{cccc}
b_{1}^{(q)}(0) & 0 & \ldots & 0 \\
\vdots & \vdots & & \vdots \\
b_{m}^{(q)}(0) & 0 & \ldots & 0
\end{array}\right) \in \mathcal{M}_{m}(\mathbb{C})
$$

In case IIa, Proposition 6.1 shows that $B_{1}, \ldots, B_{q_{0}-1}$ have real coefficients. Some coefficients of $B_{q_{0}}$ are complex.

Let $K \geq 1$. We choose the symmetrizer $\mathbf{S}$ under the form

$$
\mathbf{S}(\delta):=\sum_{q=0}^{q_{0}} \delta^{q} \mathbf{S}_{q}, \quad \mathbf{S}_{0}, \ldots, \mathbf{S}_{q_{0}-1} \in \mathcal{S}_{m}, \quad \mathbf{S}_{q_{0}} \in \mathcal{H}_{m}
$$

Computing the product of $\mathbf{S}(\delta)$ in (6.10) with $M(i \delta)$ in (6.8), then taking the real part, we first get

$$
\begin{aligned}
\operatorname{Re}(\mathbf{S}(\delta) M(i \delta))= & \operatorname{Re}\left(i \mathbf{S}_{0} N_{m}\right) \\
& +\sum_{q=1}^{q_{0}} \delta^{q} \operatorname{Re}\left(i \mathbf{S}_{q} N_{m}+i \sum_{p=0}^{q-1} \mathbf{S}_{p} B_{q-p}\right)+O\left(\delta^{q_{0}+1}\right)
\end{aligned}
$$

The choice of the real symmetric matrix $\mathbf{S}_{0}$ is the same as in [9].

Lemma 6.3 ( [9]). Let $c>0$ and let $C \in \mathbb{R}$. If $m$ is even, there exists a matrix $\mathbf{S}_{0} \in \mathcal{S}_{m}$ of the form (5.7) with $s_{1}=b_{m}^{\prime}(0)$, and that satisfies

$$
\forall W \in \mathbb{C}^{m}, \quad W^{*} \mathbf{S}_{0} W \geq C\left|W^{u}\right|^{2}-c\left|W^{s}\right|^{2} .
$$

${ }^{4}$ We feel free to use the notation $B_{1}$ even though it does not denote the same matrix as in (5.4). We hope this does not create any confusion. 
For the sake of clarity, we reproduce the proof of Lemma 6.3 in Appendix A. The main reason for doing so is that in case IId, we shall need a refined version of Lemma 6.3. We hope that the proof of this refined version will be more clear once the reader is familiar with the classical proof of Lemma 6.3.

We choose $\mathbf{S}_{0} \in \mathcal{S}_{m}$ by applying Lemma 6.3 with $c:=1 / 2$ and $C:=K^{2}+1 / 2$. It is straightforward to check that the symmetrizer $\mathbf{S}(\delta)$ in (6.10) already satisfies the properties ii), iii) of Lemma 5.7. It thus remains to fix $\mathbf{S}_{1}, \ldots, \mathbf{S}_{q_{0}}$ such that property i) of Lemma 5.7 holds.

We wish to choose the matrix $\mathbf{S}_{1} \in \mathcal{S}_{m}$ such that the coefficient of $\delta^{1}$ in the right-hand side of (6.11) vanishes. In other words, we are looking for a matrix $\mathbf{S}_{1} \in \mathcal{S}_{m}$ that satisfies

$$
\Psi\left(\mathbf{S}_{1}\right)=-\operatorname{Re}\left(i \mathbf{S}_{0} B_{1}\right) .
$$

Recall that the linear mapping $\Psi$ is defined in Lemma 6.2. The matrix $\operatorname{Re}\left(i \mathbf{S}_{0} B_{1}\right)$ is Hermitian and has purely imaginary coefficients because both $\mathbf{S}_{0}$ and $B_{1}$ belong to $\mathcal{M}_{m}(\mathbb{R})$. Applying Lemma 6.2, we can thus choose $\mathbf{S}_{1} \in \mathcal{S}_{m}$ such that (6.12) holds. Applying repeatedly Lemma 6.2, we can choose some matrices $\mathbf{S}_{2}, \ldots, \mathbf{S}_{q_{0}-1} \in \mathcal{S}_{m}$ that satisfy

$$
\forall q=2, \ldots, q_{0}-1, \quad \Psi\left(\mathbf{S}_{q}\right)+\operatorname{Re}\left(i \sum_{p=0}^{q-1} \mathbf{S}_{p} B_{q-p}\right)=0
$$

Our construction of $\mathbf{S}_{0}, \ldots, \mathbf{S}_{q_{0}-1}$ yields the following simplification in (6.11):

$$
\operatorname{Re}(\mathbf{S}(\delta) M(i \delta))=\delta^{q_{0}} \operatorname{Re}\left(i \mathbf{S}_{q_{0}} N_{m}+i \sum_{p=0}^{q_{0}-1} \mathbf{S}_{p} B_{q_{0}-p}\right)+O\left(\delta^{q_{0}+1}\right)
$$

We wish to make the coefficient of $\delta^{q_{0}}$ in (6.13) positive definite by suitably choosing $\mathbf{S}_{q_{0}} \in \mathcal{H}_{m}$. In view of Corollary 5.5, it is sufficient to check that the upper left coefficient of the matrix

$$
\operatorname{Re}\left(i \sum_{p=0}^{q_{0}-1} \mathbf{S}_{p} B_{q_{0}-p}\right)=\operatorname{Re}\left(i \mathbf{S}_{0} B_{q_{0}}\right)+\operatorname{Re}\left(i \sum_{p=1}^{q_{0}-1} \mathbf{S}_{p} B_{q_{0}-p}\right)
$$

is positive. Observing that the upper left coefficient of a matrix in $\mathcal{H}_{m} \cap i \mathcal{M}_{m}(\mathbb{R})$ is zero, we compute

$$
\operatorname{Re}\left(i \sum_{p=0}^{q_{0}-1} \mathbf{S}_{p} B_{q_{0}-p}\right)_{1,1}=\operatorname{Re}\left(i \mathbf{S}_{0} B_{q_{0}}\right)_{1,1}=\frac{-b_{m}^{\prime}(0) \operatorname{Im}\left(i^{q_{0}-1} b_{m}^{\left(q_{0}\right)}(0)\right)}{q_{0} !}
$$

The latter quantity is positive thanks to the result of Proposition 6.1. (Recall that in case IIa, $q_{0}$ is even and $\mu_{0}=0$.) Applying Corollary 5.5, there exists a matrix 
$\mathbf{S}_{q_{0}} \in \mathcal{H}_{m}$ such that the coefficient of $\delta^{q_{0}}$ in (6.13) is positive definite. Choosing $\delta$ small enough, the symmetrizer $\mathbf{S}(\delta)$ satisfies

$$
\operatorname{Re}(\mathbf{S}(\delta) M(i \delta)) \geq c \delta^{q_{0}} I
$$

for a suitable constant $c>0$. Hence property i) of Lemma 5.7 is satisfied and the result of Theorem 4.4 holds. Let us turn to case IIb.

\subsection{Case IIb}

In case IIb, we use the refined expansion

$$
M(i \delta)=i N_{m}+i \sum_{q=1}^{q_{0}} \delta^{q} B_{q}+\delta^{q_{0}+1} \beta+O\left(\delta^{q_{0}+2}\right)
$$

The matrices $B_{1}, \ldots, B_{q_{0}}$ are defined in (6.9), while $\beta$ belongs to $\mathcal{M}_{m}(\mathbb{C})$. Using Proposition 6.1, we know that $B_{1}, \ldots, B_{q_{0}-1}$ have real coefficients. Moreover the lower left coefficient of $B_{q_{0}}$ has negative imaginary part. We recall that $q_{0}$ is an odd number, with $q_{0} \geq 3$.

Let $K \geq 1$. We choose the symmetrizer $\mathbf{S}$ under the form

$$
\mathbf{S}(\delta):=\sum_{q=0}^{q_{0}+1} \delta^{q} \mathbf{S}_{q}, \quad \mathbf{S}_{0}, \ldots, \mathbf{S}_{q_{0}-2} \in \mathcal{S}_{m}, \quad \mathbf{S}_{q_{0}-1}, \mathbf{S}_{q_{0}}, \mathbf{S}_{q_{0}+1} \in \mathcal{H}_{m}
$$

Computing the product of $\mathbf{S}(\delta)$ in (6.15) with $M(i \delta)$ in (6.14), then taking the real part, we first get

$$
\begin{aligned}
\operatorname{Re}(\mathbf{S}(\delta) M(i \delta))= & \operatorname{Re}\left(i \mathbf{S}_{0} N_{m}\right)+\sum_{q=1}^{q_{0}} \delta^{q} \operatorname{Re}\left(i \mathbf{S}_{q} N_{m}+i \sum_{p=0}^{q-1} \mathbf{S}_{p} B_{q-p}\right) \\
& +\delta^{q_{0}+1} \operatorname{Re}\left(i \mathbf{S}_{q_{0}+1} N_{m}+i \sum_{p=1}^{q_{0}} \mathbf{S}_{p} B_{q_{0}+1-p}+\mathbf{S}_{0} \beta\right) \\
& +O\left(\delta^{q_{0}+2}\right) .
\end{aligned}
$$

We start as in the analysis of case IIa. We first fix $\mathbf{S}_{0} \in \mathcal{S}_{m}$ by applying Lemma 6.3 with $c:=1 / 2$ and $C:=K^{2}+1 / 2$. Then we choose $\mathbf{S}_{1}, \ldots, \mathbf{S}_{q_{0}-2} \in \mathcal{S}_{m}$ such that 
the coefficients of $\delta^{1}, \ldots, \delta^{q_{0}-2}$ in (6.16) vanish. We thus get

$$
\begin{aligned}
\operatorname{Re}(\mathbf{S}(\delta) M(i \delta))= & \delta^{q_{0}-1} \operatorname{Re}\left(i \mathbf{S}_{q_{0}-1} N_{m}+i \sum_{p=0}^{q_{0}-2} \mathbf{S}_{p} B_{q_{0}-1-p}\right) \\
& +\delta^{q_{0}} \operatorname{Re}\left(i \mathbf{S}_{q_{0}} N_{m}+i \sum_{p=0}^{q_{0}-1} \mathbf{S}_{p} B_{q_{0}-p}\right) \\
& +\delta^{q_{0}+1} \operatorname{Re}\left(i \mathbf{S}_{q_{0}+1} N_{m}+i \sum_{p=1}^{q_{0}} \mathbf{S}_{p} B_{q_{0}+1-p}+\mathbf{S}_{0} \beta\right) \\
& +O\left(\delta^{q_{0}+2}\right) .
\end{aligned}
$$

Let us now fix the matrix $\mathbf{S}_{q_{0}-1}$.

Lemma 6.4. There exists a matrix $\mathbf{S}_{q_{0}-1} \in \mathcal{H}_{m}$ that satisfies

$$
\operatorname{Re}\left(i \mathbf{S}_{q_{0}-1} N_{m}+i \sum_{p=0}^{q_{0}-2} \mathbf{S}_{p} B_{q_{0}-1-p}\right)=\left(\begin{array}{ll}
0 & 0 \\
0 & \mathbf{H}_{q_{0}-1}
\end{array}\right),
$$

where $\mathbf{H}_{q_{0}-1} \in \mathcal{H}_{\mu}$ is positive definite. Furthermore, the upper left $\mu \times \mu$ block of the matrix

$$
\sum_{p=0}^{q_{0}-1} \mathbf{S}_{p} B_{q_{0}-p}
$$

vanishes.

Proof of Lemma 6.4. Applying Lemma 6.2, we first choose a matrix $\mathbf{S}_{q_{0}-1}^{\sharp} \in \mathcal{S}_{m}$ that satisfies

$$
\operatorname{Re}\left(i \mathbf{S}_{q_{0}-1}^{\sharp} N_{m}+i \sum_{p=0}^{q_{0}-2} \mathbf{S}_{p} B_{q_{0}-1-p}\right)=0 .
$$

We decompose $\mathbf{S}_{q_{0}-1}$ as $\mathbf{S}_{q_{0}-1}=\mathbf{S}_{q_{0}-1}^{\sharp}+\mathbf{S}_{q_{0}-1}^{\mathrm{b}}$, and we are going to construct $\mathbf{S}_{q_{0}-1}^{b}$. We look for $\mathbf{S}_{q_{0}-1}^{b}$ under the form

$$
\mathbf{S}_{q_{0}-1}^{\mathrm{b}}:=\left(\begin{array}{cc}
0 & \mathbf{C}_{q_{0}-1} \\
\mathbf{C}_{q_{0}-1}^{*} & \mathbf{D}_{q_{0}-1}
\end{array}\right), \quad \mathbf{C}_{q_{0}-1} \in \mathcal{M}_{\mu}(\mathbb{C}), \quad \mathbf{D}_{q_{0}-1} \in \mathcal{H}_{\mu} .
$$

The matrix $\mathbf{C}_{q_{0}-1}$ is fixed first. Namely let us choose $\mathbf{C}_{q_{0}-1}$ of the form (5.7), that is

$$
\mathbf{C}_{q_{0}-1}:=\left(\begin{array}{cccc}
0 & \cdots & 0 & \alpha_{1} \\
\vdots & 0 & \alpha_{1} & \alpha_{2} \\
0 & \alpha_{1} & \alpha_{2} & \vdots \\
\alpha_{1} & \alpha_{2} & \cdots & \alpha_{\mu}
\end{array}\right) .
$$


We compute

$$
\begin{aligned}
\operatorname{Re}\left(i \mathbf{S}_{q_{0}-1} N_{m}+i \sum_{p=0}^{q_{0}-2} \mathbf{S}_{p} B_{q_{0}-1-p}\right) & =\operatorname{Re}\left(i \mathbf{S}_{q_{0}-1}^{b} N_{m}\right) \\
& =\left(\begin{array}{lc}
0 & 0 \\
0 & \operatorname{Re}\left(i \mathbf{D}_{q_{0}-1} N_{\mu}+i \mathbf{C}_{q_{0}-1}^{*} \tilde{N}_{\mu}\right)
\end{array}\right)
\end{aligned}
$$

where the matrix $\widetilde{N}_{\mu}$ is the same as in the decomposition (5.3). Let us also compute the upper left $\mu \times \mu$ block of the matrix (6.18). The matrices $\mathbf{S}_{0}, \ldots, \mathbf{S}_{q_{0}-2}, \mathbf{S}_{q_{0}-1}^{\sharp}$ as well as the matrices $B_{1}, \ldots, B_{q_{0}-1}$ have real coefficients. Moreover, $\mathbf{S}_{0}$ has the form (5.7) with $s_{1}=b_{m}^{\prime}(0)$. The upper left $\mu \times \mu$ block of the matrix (6.18) thus reads

$$
\mathbf{C}_{q_{0}-1} \times\left(\begin{array}{cccc}
b_{\mu+1}^{\prime}(0) & 0 & \ldots & 0 \\
\vdots & \vdots & & \vdots \\
b_{m}^{\prime}(0) & 0 & \ldots & 0
\end{array}\right)+\left(\begin{array}{cccc}
v_{1} & 0 & \ldots & 0 \\
\vdots & \vdots & & \vdots \\
v_{\mu} & 0 & \ldots & 0
\end{array}\right)
$$

where $v_{1}, \ldots, v_{\mu}$ are some complex numbers that only depend on $\mathbf{S}_{0}, \ldots, \mathbf{S}_{q_{0}-2}$ The crucial observation for what follows is that $v_{1}$ satisfies

$$
v_{1}-b_{m}^{\prime}(0) \frac{i^{q_{0}-1} b_{m}^{\left(q_{0}\right)}(0)}{q_{0} !} \in \mathbb{R} .
$$

The matrix (6.19) vanishes if and only if the coefficients $\alpha_{1}, \ldots, \alpha_{\mu}$ solve the linear system

$$
\left(\begin{array}{ccc}
b_{m}^{\prime}(0) & & 0 \\
\vdots & \ddots & \\
b_{\mu+1}^{\prime}(0) & \ldots & b_{m}^{\prime}(0)
\end{array}\right)\left(\begin{array}{c}
\alpha_{1} \\
\vdots \\
\alpha_{\mu}
\end{array}\right)=-\left(\begin{array}{c}
v_{1} \\
\vdots \\
v_{\mu}
\end{array}\right) .
$$

This system has a unique solution, which determines the matrix $\mathbf{C}_{q_{0}-1} \in \mathcal{M}_{\mu}(\mathbb{C})$. In particular, (6.20) and Proposition 6.1 show that $\alpha_{1}=-v_{1} / b_{m}^{\prime}(0)$ has positive imaginary part.

It remains to fix the matrix $\mathbf{D}_{q_{0}-1} \in \mathcal{H}_{\mu}$. Using the expression above for the matrix $\mathbf{C}_{q_{0}-1}$, the upper left coefficient of $\operatorname{Re}\left(i \mathbf{C}_{q_{0}-1}^{*} \tilde{N}_{\mu}\right)$ equals $\operatorname{Im} \alpha_{1}>0$. Consequently we can apply Corollary 5.5 and find $\mathbf{D}_{q_{0}-1} \in \mathcal{H}_{\mu}$ such that

$$
\operatorname{Re}\left(i \mathbf{D}_{q_{0}-1} N_{\mu}+i \mathbf{C}_{q_{0}-1}^{*} \tilde{N}_{\mu}\right)
$$

is positive definite. This completes the proof of Lemma 6.4.

We now fix the matrices $\mathbf{S}_{q_{0}}$ and $\mathbf{S}_{q_{0}+1}$. We choose $\mathbf{S}_{q_{0}} \in \mathcal{H}_{m}$ of the form

$$
\mathbf{S}_{q_{0}}:=\left(\begin{array}{cc}
0 & \mathbf{C}_{q_{0}} \\
\mathbf{C}_{q_{0}}^{*} & 0
\end{array}\right), \quad \mathbf{C}_{q_{0}}:=\left(\begin{array}{cc}
0 & \mathfrak{c} \\
& \mathfrak{c} \\
\mathfrak{c} & 0
\end{array}\right) \in \mathcal{M}_{\mu}(\mathbb{C})
$$


and we choose $\mathbf{S}_{q_{0}+1} \in \mathcal{H}_{m}$ of the form

$$
\mathbf{S}_{q_{0}+1}:=\left(\begin{array}{cc}
\mathbf{A}_{q_{0}+1} & 0 \\
0 & 0
\end{array}\right), \quad \mathbf{A}_{q_{0}+1} \in \mathcal{H}_{\mu} .
$$

We compute

$$
\operatorname{Re}\left(i \mathbf{S}_{q_{0}} N_{m}\right)=\left(\begin{array}{lc}
0 & 0 \\
0 \operatorname{Re}\left(i \mathbf{C}_{q_{0}}^{*}\right. & \left.\tilde{N}_{\mu}\right)
\end{array}\right) .
$$

Using the latter relation as well as Lemma 6.4 yields some simplifications in (6.17). At this stage, we have

$$
\begin{aligned}
& \operatorname{Re}(\mathbf{S}(\delta) M(i \delta))=\delta^{q_{0}-1}\left(\begin{array}{ll}
0 & 0 \\
0 & \mathbf{H}_{q_{0}-1}
\end{array}\right)+\delta^{q_{0}}\left(\begin{array}{cc}
0 & \mathbf{H}_{q_{0}} \\
\mathbf{H}_{q_{0}}^{*} & 0
\end{array}\right) \\
& \quad+\delta^{q_{0}+1}\left(\begin{array}{cc}
\operatorname{Re}\left(i \mathbf{S}_{q_{0}+1} N_{m}+i \sum_{p=1}^{q_{0}} \mathbf{S}_{p} B_{q_{0}+1-p}+\mathbf{S}_{0} \beta\right)_{1 \ldots \mu, 1 \ldots \mu} & 0 \\
0 & 0
\end{array}\right) \\
& \quad+\left(\begin{array}{cc}
O\left(\delta^{q_{0}+2}\right) & O\left(\delta^{q_{0}+1}\right) \\
O\left(\delta^{q_{0}+1}\right) & O\left(\delta^{q_{0}}\right)
\end{array}\right),
\end{aligned}
$$

where $\mathbf{H}_{q_{0}-1} \in \mathcal{H}_{m}$ is positive definite, and $\mathbf{H}_{q_{0}} \in \mathcal{M}_{m}(\mathbb{C})$ does not depend on $\mathfrak{c}$ nor on $\mathbf{A}_{q_{0}+1}$.

Following the same strategy as in the proof of Lemma 5.6, we can choose $\mathfrak{c} \in \mathbb{C}$ and $\mathbf{A}_{q_{0}+1} \in \mathcal{H}_{\mu}$ such that the block

$$
\left(\operatorname{Re}\left(i \mathbf{S}_{q_{0}+1} N_{m}\right)+\operatorname{Re}\left(i \mathbf{S}_{q_{0}} B_{1}\right)\right)_{1 \ldots \mu, 1 \ldots \mu}
$$

is positive definite as large as we wish. We can now conclude as in the analysis of case I. Applying Young's inequality and choosing $\delta$ small enough, the symmetrizer $\mathbf{S}(\delta)$ satisfies

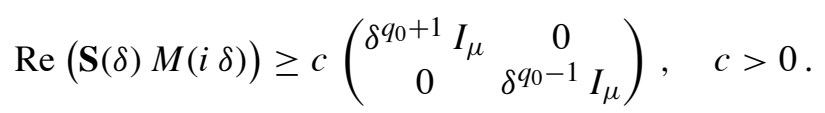

Hence $\mathbf{S}(\delta)$ satisfies properties i), ii), iii) of Lemma 5.7, and the result of Theorem 4.4 holds. Let us now turn to the more involved case IIc.

\subsection{Case IIc}

The following result is similar to Lemma 6.2 and will be used in the analysis below.

Lemma 6.5. Let $v \geq 1$ be an integer, and let $\Phi$ be the linear mapping defined by

$$
\begin{aligned}
\Phi: \mathcal{H}_{v} & \longrightarrow \mathcal{H}_{v} \\
S & \longmapsto \operatorname{Re}\left(i S N_{v}\right) .
\end{aligned}
$$


The kernel of $\Phi$ is made of all matrices $S \in \mathcal{S}_{\nu}$ of the form (5.7). If $v$ is even, the image of $\Phi$ is made of all matrices $H \in \mathcal{H}_{v}$ that satisfy

$$
\forall j=1, \ldots, \frac{v}{2}, \quad \frac{1}{2} H_{j, j}+\operatorname{Re}\left(\sum_{k=1}^{j-1} H_{j-k, j+k}\right)=\operatorname{Re}\left(\sum_{k=0}^{j-1} H_{j-k, j+k+1}\right)=0 .
$$

If $v$ is odd, the image of $\Phi$ is made of all matrices $H \in \mathcal{H}_{v}$ that satisfy

$$
\begin{aligned}
\forall j=1, \ldots, \frac{\nu+1}{2}, \quad \frac{1}{2} H_{j, j}+\operatorname{Re}\left(\sum_{k=1}^{j-1} H_{j-k, j+k}\right) & =0, \\
\forall j=1, \ldots, \frac{\nu-1}{2}, \quad \operatorname{Re}\left(\sum_{k=0}^{j-1} H_{j-k, j+k+1}\right) & =0 .
\end{aligned}
$$

Proof of Lemma 6.5. The dimension of the (real) vector space $\mathcal{H}_{v}$ is $v^{2}$. Lemma 5.2 shows that the kernel of $\Phi$ is made of all matrices $S \in \mathcal{S}_{\nu}$ of the form (5.7), and thus coincides with the kernel of $\Psi$. Consequently the rank of $\Phi$ is $v^{2}-v$.

It is not so difficult to check that if $v$ is even, then any matrix $H$ in the image of $\Phi$ satisfies the conditions (6.21). Moreover, the Hermitian matrices that satisfy (6.21) form a subspace of $\mathcal{H}_{v}$ of dimension $v^{2}-v$. The same arguments yield the characterization of the image of $\Phi$ when $v$ is odd.

We shall also need the following rectangular version of Lemma 5.2. The proof is completely elementary and therefore omitted.

Lemma 6.6. Let $v_{1} \geq 1$ and $v_{2}>v_{1}$ be some integers. A matrix $S \in \mathcal{M}_{v_{1}, v_{2}}(\mathbb{C})$ satisfies $S N_{v_{2}}=N_{\nu_{1}}^{*} S$ if and only if $S$ has the form

$$
S=\left(\begin{array}{ccccccc}
0 & \ldots & 0 & 0 & \cdots & 0 & s_{1} \\
\vdots & & \vdots & \vdots & 0 & s_{1} & s_{2} \\
\vdots & & \vdots & 0 & s_{1} & s_{2} & \vdots \\
0 & \ldots & 0 & s_{1} & s_{2} & \cdots & s_{v_{1}}
\end{array}\right)
$$

Let us now construct a symmetrizer $\mathbf{S}(\delta)$ in case IIc. We recall that $q_{0}$ is even and the remainder $\mu_{0}$ in the Euclidean division (6.1) satisfies $1 \leq \mu_{0} \leq \mu-1$. In particular there holds $\mu \geq 2$, that is $m \geq 4$. We expand $M(i \delta)$ as

$$
M(i \delta)=i N_{m}+i \sum_{q=1}^{q_{0}} \delta^{q} B_{q}+\delta^{q_{0}+1} \beta_{1}+\delta^{q_{0}+2} \beta_{2}+O\left(\delta^{q_{0}+3}\right) .
$$

The matrices $B_{1}, \ldots, B_{q_{0}}$ are defined in (6.9), while the matrices $\beta_{1}, \beta_{2}$ belong to $\mathcal{M}_{m}(\mathbb{C})$ and have the same form as $B_{1}, \ldots, B_{q_{0}}$ (only the first column is nonzero). Applying Proposition 6.1, we know that $B_{1}, \ldots, B_{q_{0}-1}$ have real coefficients. 
Moreover the coefficients of $B_{q_{0}}$ satisfy

$$
i^{q_{0}-1} b_{m}^{\left(q_{0}\right)}(0), \ldots, i^{q_{0}-1} b_{m-2 \mu_{0}+1}^{\left(q_{0}\right)}(0) \in \mathbb{R}, \quad b_{m}^{\prime}(0) \operatorname{Im}\left(i^{q_{0}-1} b_{m-2 \mu_{0}}^{\left(q_{0}\right)}(0)\right)<0 \text {. }
$$

We have absolutely no information on $b_{m-2 \mu_{0}-1}^{\left(q_{0}\right)}(0), \ldots, b_{1}^{\left(q_{0}\right)}(0)$, nor on $\beta_{1}, \beta_{2}$.

Let $K \geq 1$. We seek the symmetrizer $\mathbf{S}$ under the form

$$
\mathbf{S}(\delta):=\sum_{q=0}^{q_{0}+2} \delta^{q} \mathbf{S}_{q}, \quad \mathbf{S}_{0}, \ldots, \mathbf{S}_{q_{0}-1} \in \mathcal{S}_{m}, \quad \mathbf{S}_{q_{0}}, \mathbf{S}_{q_{0}+1}, \mathbf{S}_{q_{0}+2} \in \mathcal{H}_{m}
$$

Computing the product of $\mathbf{S}(\delta)$ in (6.27) with $M(i \delta)$ in (6.25) and taking the real part yields

$$
\begin{aligned}
& \operatorname{Re}(\mathbf{S}(\delta) M(i \delta))=\operatorname{Re}\left(i \mathbf{S}_{0} N_{m}\right)+\sum_{q=1}^{q_{0}} \delta^{q} \operatorname{Re}\left(i \mathbf{S}_{q} N_{m}+i \sum_{p=0}^{q-1} \mathbf{S}_{p} B_{q-p}\right) \\
& \quad+\delta^{q_{0}+1} \operatorname{Re}\left(i \mathbf{S}_{q_{0}+1} N_{m}+i \sum_{p=1}^{q_{0}} \mathbf{S}_{p} B_{q_{0}+1-p}+\mathbf{S}_{0} \beta_{1}\right) \\
& \quad+\delta^{q_{0}+2} \operatorname{Re}\left(i \mathbf{S}_{q_{0}+2} N_{m}+i \sum_{p=2}^{q_{0}+1} \mathbf{S}_{p} B_{q_{0}+2-p}+\mathbf{S}_{1} \beta_{1}+\mathbf{S}_{0} \beta_{2}\right) \\
& \quad+O\left(\delta^{q_{0}+2}\right) .
\end{aligned}
$$

The starting point is the same as in cases IIa and IIb. We choose $\mathbf{S}_{0} \in \mathcal{S}_{m}$ by applying Lemma 6.3 with $c:=1 / 2$ and $C:=K^{2}+1 / 2$. Properties ii), iii) of Lemma 5.7 are satisfied. Then we apply Lemma 6.2 and choose $\mathbf{S}_{1}, \ldots, \mathbf{S}_{q_{0}-1} \in$ $\mathcal{S}_{m}$ such that the coefficients of $\delta^{1}, \ldots, \delta^{q_{0}-1}$ in the right-hand side of (6.28) vanish. The choice of the matrix $\mathbf{S}_{q_{0}}$ is more delicate.

Lemma 6.7. There exists a matrix $\mathbf{S}_{q_{0}} \in \mathcal{H}_{m}$ that satisfies

$$
\operatorname{Re}\left(i \mathbf{S}_{q_{0}} N_{m}+i \sum_{p=0}^{q_{0}-1} \mathbf{S}_{p} B_{q_{0}-p}\right)=\left(\begin{array}{cc}
0 & 0 \\
0 & \mathbf{H}_{q_{0}}
\end{array}\right)
$$

where $\mathbf{H}_{q_{0}} \in \mathcal{H}_{m-\mu_{0}}$ is positive definite. Furthermore, the upper left $\mu_{0} \times \mu_{0}$ block of the matrix

$$
\sum_{p=1}^{q_{0}} \mathbf{S}_{p} B_{q_{0}+1-p}-i \mathbf{S}_{0} \beta_{1}
$$

vanishes. 
Proof of Lemma 6.7. The difficulty lies in the fact that the product $\mathbf{S}_{0} B_{q_{0}}$ appears in the left-hand side of (6.29) and we do not know how all the coefficients of $B_{q_{0}}$ look like. More precisely, we know that $B_{q_{0}}$ has the form (6.9) and its coefficients satisfy (6.26). The matrix $\mathbf{S}_{0}$ has the form (5.7) with $s_{1}=b_{m}^{\prime}(0)$. Therefore we compute

$$
\operatorname{Re}\left(i \mathbf{S}_{0} B_{q_{0}}\right)=\frac{1}{2}\left(\begin{array}{cccc}
0 & -i \overline{v_{2}} & \ldots & -i \overline{v_{m}} \\
i v_{2} & 0 & \ldots & 0 \\
\vdots & \vdots & & \vdots \\
i v_{m} & 0 & \ldots & 0
\end{array}\right) .
$$

The numbers $v_{2}, \ldots, v_{m}$ in (6.31) satisfy

$$
v_{2}, \ldots, v_{2} \mu_{0} \in \mathbb{R}, \quad \operatorname{Im} v_{2} \mu_{0}+1<0 .
$$

We have no information on $v_{2} \mu_{0}+2, \ldots, v_{m}$. We decompose the matrix in (6.31) as

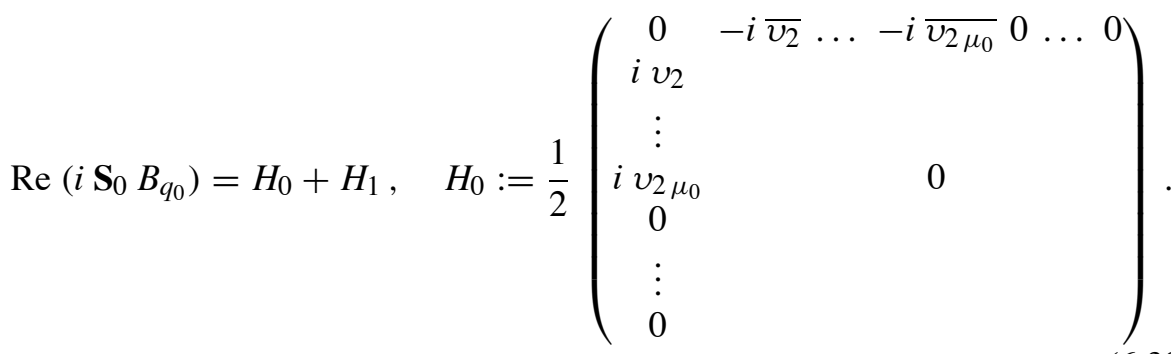

In particular, the matrix $H_{0}$ is Hermitian with purely imaginary coefficients.

Using Lemma 6.2, we first choose a matrix $\mathbf{S}_{q_{0}}^{\sharp} \in \mathcal{S}_{m}$ that satisfies

$$
\operatorname{Re}\left(i \mathbf{S}_{q_{0}}^{\sharp} N_{m}+i \sum_{p=1}^{q_{0}-1} \mathbf{S}_{p} B_{q_{0}-p}\right)+H_{0}=0 .
$$

This is possible because the matrices $\mathbf{S}_{1}, \ldots, \mathbf{S}_{q_{0}-1}, B_{1}, \ldots, B_{q_{0}-1}$ have real coefficients.

The Hermitian matrix $H_{1}$ is the difference between the matrix $\operatorname{Re}\left(i \mathbf{S}_{0} B_{q_{0}}\right)$ whose expression is given in (6.31), and the matrix $H_{0}$ whose expression is given in (6.33). At this stage, the reader can check that $H_{1}$ does not belong to the image of the linear mapping $\Phi$ (see the conditions (6.21) that characterize the image of $\Phi$ ). Let us construct a matrix $H_{2} \in \mathcal{H}_{m}$ such that $H_{1}-H_{2}$ belongs to the image of $\Phi$. We choose $\mathrm{H}_{2}$ of the form

$$
H_{2}:=\left(\begin{array}{cc}
0 & 0 \\
0 & \widetilde{H}_{2}
\end{array}\right), \quad \widetilde{H}_{2} \in \mathcal{H}_{m-\mu_{0}} .
$$

The matrix $\widetilde{H}_{2} \in \mathcal{H}_{m-\mu_{0}}$ is defined in the following way:

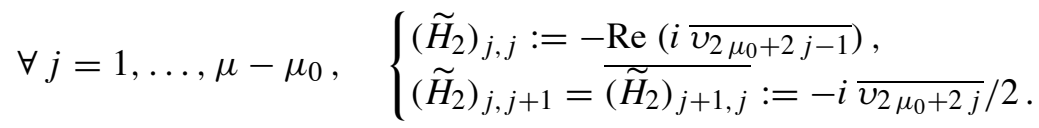


All other coefficients in $\widetilde{H}_{2}$ vanish. Straightforward computations show that $H_{1}-$ $\mathrm{H}_{2}$ satisfies conditions (6.21) and thus belongs to the image of $\Phi$. Moreover, the upper left coefficient of $\widetilde{H}_{2}$ is positive (use (6.35) and (6.32)). We can choose $\mathbf{S}_{q_{0}}^{\mathrm{b}} \in \mathcal{H}_{m}$ that satisfies

$$
\Phi\left(\mathbf{S}_{q_{0}}^{b}\right)+H_{1}-H_{2}=0 .
$$

It remains to choose $\mathbf{S}_{q_{0}}$ under the form

$$
\mathbf{S}_{q_{0}}:=\mathbf{S}_{q_{0}}^{\sharp}+\mathbf{S}_{q_{0}}^{b}+\mathbf{S}_{q_{0}}^{\natural},
$$

where the matrix $\mathbf{S}_{q_{0}}^{\natural}$ has the structure

$$
\mathbf{S}_{q_{0}}^{\natural}:=\left(\begin{array}{cc}
0 & \mathbf{C}_{q_{0}} \\
\mathbf{C}_{q_{0}}^{*} & \mathbf{D}_{q_{0}}
\end{array}\right), \quad \mathbf{C}_{q_{0}} \in \mathcal{M}_{\mu_{0}, m-\mu_{0}}(\mathbb{C}), \quad \mathbf{D}_{q_{0}} \in \mathcal{H}_{m-\mu_{0}}
$$

The matrix $\mathbf{C}_{q_{0}}$ is chosen of the form (6.24), that is

$$
\mathbf{C}_{q_{0}}:=\left(\begin{array}{ccccccc}
0 & \ldots & 0 & 0 & \cdots & 0 & \Theta_{1} \\
\vdots & & \vdots & \vdots & 0 & \Theta_{1} & \Theta_{2} \\
\vdots & & \vdots & 0 & \Theta_{1} & \Theta_{2} & \vdots \\
0 & \ldots & 0 & \Theta_{1} & \Theta_{2} & \cdots & \Theta_{\mu_{0}}
\end{array}\right) \in \mathcal{M}_{\mu_{0}, m-\mu_{0}}(\mathbb{C})
$$

The upper left $\mu_{0} \times \mu_{0}$ block of the matrix (6.30) then reads ${ }^{5}$

$$
\mathbf{C}_{q_{0}} \times \underbrace{\left(\begin{array}{cccc}
b_{\mu_{0}+1}^{\prime}(0) & 0 & \ldots & 0 \\
\vdots & \vdots & & \vdots \\
b_{m}^{\prime}(0) & 0 & \ldots & 0
\end{array}\right)}_{\in \mathcal{M}_{m-\mu_{0}, \mu_{0}}(\mathbb{R})}+\underbrace{\left(\begin{array}{cccc}
\Upsilon_{1} & 0 & \ldots & 0 \\
\vdots & \vdots & & \vdots \\
\Upsilon_{\mu_{0}} & 0 & \ldots & 0
\end{array}\right)}_{\in \mathcal{M}_{\mu_{0}}(\mathbb{C})}
$$

where the coefficients $\Upsilon_{1}, \ldots, \Upsilon_{\mu_{0}}$ are determined by the matrices $\mathbf{S}_{0}, \ldots, \mathbf{S}_{q_{0}-1}$, $\mathbf{S}_{q_{0}}^{\sharp}, \mathbf{S}_{q_{0}}^{\mathrm{b}}$ which have already been fixed. It is clear that there exists a unique choice of the coefficients $\Theta_{1}, \ldots, \Theta_{\mu_{0}}$ such that this upper left block vanishes. These coefficients are determined by solving an invertible linear system of dimension $\mu_{0}$.

It remains to fix $\mathbf{D}_{q_{0}}$ such that (6.29) holds with a positive definite $\mathbf{H}_{q_{0}}$. Let us compute the matrix in the left-hand side of (6.29) by using the relations (6.34) and

${ }^{5}$ We recall that only the first column of the matrices $B_{1}, \ldots, B_{q_{0}}, \beta_{1}$ does not vanish. 
(6.36). We have

$$
\begin{aligned}
\operatorname{Re}\left(i \mathbf{S}_{q_{0}} N_{m}+i \sum_{p=0}^{q_{0}-1} \mathbf{S}_{p} B_{q_{0}-p}\right) \\
=\operatorname{Re}\left(i \mathbf{S}_{q_{0}}^{\natural} N_{m}\right)+H_{2} \\
\quad+\operatorname{Re}\left(i \mathbf{S}_{q_{0}}^{b} N_{m}\right)+H_{1}-H_{2} \\
\quad+\operatorname{Re}\left(i \mathbf{S}_{q_{0}}^{\sharp} N_{m}\right)+H_{0}+\operatorname{Re}\left(i \sum_{p=1}^{q_{0}-1} \mathbf{S}_{p} B_{q_{0}-p}\right) \\
=\operatorname{Re}\left(i \mathbf{S}_{q_{0}}^{\natural} N_{m}\right)+H_{2} .
\end{aligned}
$$

Following the block decomposition (6.37) of $\mathbf{S}_{q_{0}}^{\natural}$, we introduce the block decomposition of $N_{m}$, that is

$$
N_{m}=\left(\begin{array}{cc}
N_{\mu_{0}} & \tilde{N}_{\mu_{0}, m-\mu_{0}} \\
0 & N_{m-\mu_{0}}
\end{array}\right) .
$$

Going back to (6.38), we compute the real part of $i \mathbf{S}_{q_{0}}^{\natural} N_{m}$ by applying Lemma 6.6. We thus obtain

$$
\operatorname{Re}\left(i \mathbf{S}_{q_{0}} N_{m}+i \sum_{p=0}^{q_{0}-1} \mathbf{S}_{p} B_{q_{0}-p}\right)=\left(\begin{array}{ll}
0 & \tilde{H}_{2}+\operatorname{Re}\left(i \mathbf{C}_{q_{0}}^{*} \tilde{N}_{\mu_{0}, m-\mu_{0}}+i \mathbf{D}_{q_{0}} N_{m-\mu_{0}}\right) \\
0 & 0
\end{array}\right)
$$

The first row of $\mathbf{C}_{q_{0}}^{*}$ is zero so the upper left coefficient of $\operatorname{Re}\left(i \mathbf{C}_{q_{0}}^{*} \widetilde{N}_{\mu_{0}, m-\mu_{0}}\right)$ is zero. Consequently, the upper left coefficient of $\widetilde{H}_{2}+\operatorname{Re}\left(i \mathbf{C}_{q_{0}}^{*} \widetilde{N}_{\mu_{0}, m-\mu_{0}}\right)$ is positive. Applying Corollary 5.5, we can choose $\mathbf{D}_{q_{0}} \in \mathcal{H}_{m-\mu_{0}}$ such that the lower right block in (6.29) is positive definite. form

It remains to fix the matrices $\mathbf{S}_{q_{0}+1}$ and $\mathbf{S}_{q_{0}+2}$. We first choose $\mathbf{S}_{q_{0}+1}$ of the

$$
\mathbf{S}_{q_{0}+1}:=\left(\begin{array}{cc}
0 & \mathbf{C}_{q_{0}+1} \\
\mathbf{C}_{q_{0}+1}^{*} & 0
\end{array}\right), \quad \mathbf{C}_{q_{0}+1} \in \mathcal{M}_{\mu_{0}, m-\mu_{0}}(\mathbb{C}),
$$

and $\mathbf{S}_{q_{0}+2}$ of the form

$$
\mathbf{S}_{q_{0}+2}:=\left(\begin{array}{cc}
\mathbf{A}_{q_{0}+2} & 0 \\
0 & 0
\end{array}\right), \quad \mathbf{A}_{q_{0}+2} \in \mathcal{H}_{\mu_{0}}
$$

We choose $\mathbf{C}_{q_{0}+1}$ of the form (6.24). Lemma 6.6 shows that the coefficient of $\delta^{q_{0}+1}$ in (6.29) has the form

$$
\left(\begin{array}{cc}
0 & \mathbf{H}_{q_{0}+1} \\
\mathbf{H}_{q_{0}+1}^{*} & \star
\end{array}\right)
$$


where $\mathbf{H}_{q_{0}+1} \in \mathcal{M}_{\mu_{0}, m-\mu_{0}}(\mathbb{C})$ does not depend on $\mathbf{C}_{q_{0}+1}$. To conclude the construction of $\mathbf{S}$, it remains to observe that we can choose $\mathbf{C}_{q_{0}+1}$ of the form (6.24) and $\mathbf{A}_{q_{0}+2} \in \mathcal{H}_{\mu_{0}}$ so that the upper left $\mu_{0} \times \mu_{0}$ block of

$$
\operatorname{Re}\left(i \mathbf{S}_{q_{0}+2} N_{m}+i \mathbf{S}_{q_{0}+1} B_{1}\right)
$$

is positive definite as large as we wish. (The argument is entirely similar to Lemma 5.6 so we do not repeat it.) Young's inequality and the argument already used for case I and case IIb show that we can achieve the estimate

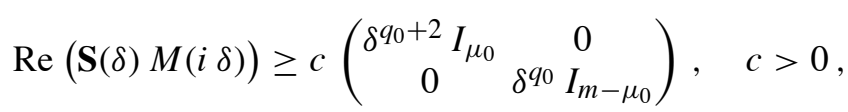

provided that $\delta$ is small enough. The symmetrizer $\mathbf{S}(\delta)$ thus satifies all properties of Lemma 5.7 and Theorem 4.4 holds. We now turn to case IId, which still requires some new arguments.

\subsection{Case IId}

In this paragraph, $q_{0}$ is odd and $\mu_{0}$ satisfies $1 \leq \mu_{0} \leq \mu-1$. In particular, we have $\mu \geq 2$. The case $q_{0}=1$ has to be dealt with separately from the case $q_{0} \geq 3$ because the value of the symmetrizer $\mathbf{S}$ at the origin is different. Let us therefore deal first with the case $q_{0}=1$. Applying Proposition 6.1, we know that $b_{m}^{\prime}(0), \ldots, b_{m-2 \mu_{0}+1}^{\prime}(0)$ are real numbers while $b_{m-2 \mu_{0}}^{\prime}(0)$ has negative imaginary part. We expand $M(i \delta)$ as

$$
M(i \delta)=i N_{m}+i \delta B_{1}+\delta^{2} \beta+O\left(\delta^{3}\right), \quad B_{1}:=\left(\begin{array}{cccc}
b_{1}^{\prime}(0) & 0 & \ldots & 0 \\
\vdots & \vdots & & \vdots \\
b_{m}^{\prime}(0) & 0 & \ldots & 0
\end{array}\right)
$$

and we choose the symmetrizer $\mathbf{S}$ under the form

$$
\mathbf{S}(\delta)=\mathbf{S}_{0}+\delta \mathbf{S}_{1}+\delta^{2} \mathbf{S}_{2}, \quad \mathbf{S}_{0}, \mathbf{S}_{1}, \mathbf{S}_{2} \in \mathcal{H}_{m}
$$

We fix a constant $K \geq 1$. As usual, the matrix $\mathbf{S}_{0}$ is determined first.

Lemma 6.8. There exists a matrix $\mathbf{S}_{0} \in \mathcal{H}_{m}$ that satisfies

- for all $W \in \mathbb{C}^{m}, W^{*} \mathbf{S}_{0} W \geq\left(K^{2}+1 / 2\right)\left|W^{u}\right|^{2}-\left|W^{s}\right|^{2} / 2$,

- the upper left coefficient of $\operatorname{Re}\left(\mathbf{S}_{0} M^{\prime}(0)\right)$ is positive,

- the matrix $\operatorname{Re}\left(i \mathbf{S}_{0} N_{m}\right)$ has the form

$$
\left(\begin{array}{cc}
0 & 0 \\
0 & \mathbf{H}_{\mathbf{0}}
\end{array}\right), \quad \mathbf{H}_{\mathbf{0}} \in \mathcal{H}_{\mu-\mu_{0}},
$$

where $\mathbf{H}_{\mathbf{0}}$ is positive definite, 
- the upper left $\left(\mu+\mu_{0}\right) \times\left(\mu+\mu_{0}\right)$ block of $\operatorname{Re}\left(i \mathbf{S}_{0} B_{1}\right)$ has purely imaginary coefficients.

Proof of Lemma 6.8. The idea consists in "interpolating" between the construction of $\mathbf{S}(0)$ in case I and the classical construction by Kreiss (see Lemma 6.3 whose proof is reproduced in Appendix A). More precisely, let us consider some real numbers $c_{1}, \ldots, c_{2} \mu_{0}$, some complex numbers $c_{2} \mu_{0}+1, \ldots, c_{\mu+\mu_{0}}$ and a matrix $\widetilde{\mathbf{D}}_{0} \in \mathcal{H}_{\mu-\mu_{0}}$ to be fixed later on. We choose $\mathbf{S}_{0}$ of the form

$$
\mathbf{S}_{0}:=\left(\begin{array}{cc}
0 & \mathbf{C}_{0} \\
\mathbf{C}_{0}^{*} & \mathbf{D}_{\mathbf{0}}
\end{array}\right)
$$

where the matrices $\mathbf{C}_{0} \in \mathcal{M}_{\mu}(\mathbb{C})$ and $\mathbf{D}_{0} \in \mathcal{H}_{\mu}$ are defined as follows:

$$
\mathbf{C}_{0}:=\left(\begin{array}{cccc}
0 & \cdots & 0 & c_{1} \\
\vdots & 0 & c_{1} & c_{2} \\
0 & c_{1} & c_{2} & \vdots \\
c_{1} & c_{2} & \cdots & c_{\mu}
\end{array}\right), \quad \mathbf{D}_{0}:=\left(\begin{array}{cccccc}
c_{2} & \ldots & c_{\mu_{0}+1} & c_{\mu_{0}+2} & \ldots & c_{\mu+1} \\
\ddots & \vdots & \vdots & & \vdots \\
& c_{2} \mu_{0} & c_{2} \mu_{0}+1 & \ldots & c_{\mu+\mu_{0}} \\
\star & & & \widetilde{\mathbf{D}}_{0} &
\end{array}\right) .
$$

The $\star$ symbol in the definition of $\mathbf{D}_{0}$ stands for the unique coefficients that make $\mathbf{D}_{0}$ a Hermitian matrix.

For a vector $W=\left(W_{1}, \ldots, W_{m}\right) \in \mathbb{C}^{m}$, we recall that $W^{s}$ denotes the vector $\left(W_{1}, \ldots, W_{\mu}\right), W^{u}$ denotes the vector $\left(W_{\mu+1}, \ldots, W_{m}\right)$. We also introduce the notation $\widetilde{W}$ to denote the vector $\left(W_{\mu+\mu_{0}+1}, \ldots, W_{m}\right) \in \mathbb{C}^{\mu-\mu_{0}}$. We compute

$$
W^{*} \mathbf{S}_{0} W=\sum_{k=1}^{2 \mu_{0}} c_{k} \sum_{j=k}^{m} \bar{W}_{j} W_{m+k-j}+\sum_{k=2 \mu_{0}+1}^{\mu+\mu_{0}} \sum_{\substack{j=k \\ j<m+k-j}}^{m} 2 \operatorname{Re}\left(c_{k} \bar{W}_{j} W_{m+k-j}\right)+\widetilde{W}^{*} \widetilde{D}_{0} \widetilde{W} .
$$

We first choose $c_{1}:=b_{m}^{\prime}(0)$ so the upper left coefficient of $\operatorname{Re}\left(\mathbf{S}_{0} M^{\prime}(0)\right)$ equals $b_{m}^{\prime}(0)^{2}>0$. Following the proof of Lemma 6.3, we also choose $c_{3}=\cdots=$ $c_{2} \mu_{0}-1:=0$. Using the same arguments as in the proof of Lemma 6.3 (see Appendix A), we can choose inductively $c_{2}, \ldots, c_{2} \mu_{0}>0$ sufficiently large such that, for a certain constant $C_{0}>0$, there holds

$$
\begin{aligned}
W^{*} \mathbf{S}_{0} W \geq & -\frac{1}{4}\left|W^{s}\right|^{2}+\left(K^{2}+1\right) \sum_{k=1}^{\mu_{0}}\left|W_{\mu+k}\right|^{2}-C_{0}|\widetilde{W}|^{2} \\
& +\sum_{\substack{j=k \\
j<m+k-j}}^{m} 2 \operatorname{Re}\left(c_{k} \bar{W}_{j} W_{m+k-j}\right)+\widetilde{W}^{*} \widetilde{D}_{0} \widetilde{W} .
\end{aligned}
$$

The constant $C_{0}$ in (6.41) depends on $K$ and $b_{m}^{\prime}(0)$ but it does not depend on $c_{2 \mu_{0}+1}, \ldots, c_{\mu+\mu_{0}}$ nor on $\widetilde{D}_{0}$. 
We now choose the coefficients $c_{2} \mu_{0}+1, \ldots, c_{\mu+\mu_{0}}$ as the unique solution to the triangular linear system

$$
\begin{aligned}
&\left(\begin{array}{ccc}
b_{m}^{\prime}(0) & & 0 \\
\vdots & \ddots & \\
b_{\mu+\mu_{0}+1}^{\prime}(0) & \cdots & b_{m}^{\prime}(0)
\end{array}\right)\left(\begin{array}{c}
c_{2} \mu_{0}+1 \\
\vdots \\
c_{\mu+\mu_{0}}
\end{array}\right) \\
&=-\left(\begin{array}{c}
c_{1} b_{m-2 \mu_{0}}^{\prime}(0)+\cdots+c_{2 \mu_{0}} b_{m-1}^{\prime}(0) \\
\vdots \\
c_{1} b_{\mu-\mu_{0}+1}^{\prime}(0)+\cdots+c_{2 \mu_{0}} b_{\mu+\mu_{0}}^{\prime}(0)
\end{array}\right) .
\end{aligned}
$$

This choice has two consequences. First, $c_{2} \mu_{0}+1$ has positive imaginary part. Second, the reader can check that the upper left $\left(\mu+\mu_{0}\right) \times\left(\mu+\mu_{0}\right)$ block of $\operatorname{Re}\left(i \mathbf{S}_{0} B_{1}\right)$ has purely imaginary coefficients.

Let us now go back to the estimate (6.41). Since $c_{2} \mu_{0}+1, \ldots, c_{\mu+\mu_{0}}$ are fixed, we wish to apply Young's inequality for the cross products in the right-hand side of (6.41). More precisely, let us consider a product $c_{k} \bar{W}_{j} W_{m+k-j}$ with $k=2 \mu_{0}+$ $1, \ldots, \mu+\mu_{0}$ and $j \geq k, j<m+k-j$. Then at least one of the indices $j$, $m+k-j$ is strictly larger than $\mu+\mu_{0}$. It may happen that both are strictly larger than $\mu+\mu_{Q}$. Consequently, we need to derive a lower bound for terms of the form $\left|W^{s}\right||W|,\left|\left(W_{\mu+1}, \ldots, W_{\mu+\mu_{0}}\right)\right||\widetilde{W}|$ or $|\widetilde{W}|^{2}$. We apply Young's inequality in (6.41) and obtain

$$
W^{*} \mathbf{S}_{0} W \geq-\frac{1}{2}\left|W^{s}\right|^{2}+\left(K^{2}+\frac{1}{2}\right) \sum_{k=1}^{\mu_{0}}\left|W_{\mu+k}\right|^{2}+\widetilde{W}^{*}\left(\widetilde{D}_{0}-C_{1} I\right) \tilde{W},
$$

where $C_{1}>C_{0}$ is a new constant that does not depend on $\widetilde{D}_{0}$.

With our choice of $\mathbf{S}_{0}$, we can compute

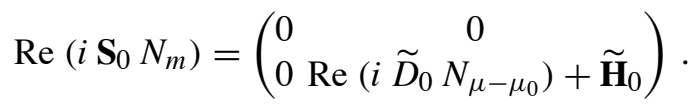

The upper left coefficient of $\widetilde{\mathbf{H}}_{0}$ equals Im $c_{2} \mu_{0}+1>0$. Applying Lemma 5.4, we can choose a matrix $\widetilde{\mathbf{D}}_{0} \in \mathcal{H}_{\mu-\mu_{0}}$ that satisfies

$$
\widetilde{D}_{0} \geq\left(C_{1}+K^{2}+\frac{1}{2}\right) I,
$$

and such that $\operatorname{Re}\left(i \widetilde{D}_{0} N_{\mu-\mu_{0}}\right)+\widetilde{\mathbf{H}}_{0}$ is positive definite. We have thus constructed a matrix $\mathbf{S}_{0} \in \mathcal{H}_{m}$ that satisfies all the properties stated in Lemma 6.8.

We now need to fix the matrices $\mathbf{S}_{1}, \mathbf{S}_{2}$. The choice of $\mathbf{S}_{0}$ yields

$$
\begin{aligned}
\operatorname{Re}(i \mathbf{S}(\delta) M(i \delta))= & \left(\begin{array}{cc}
0 & 0 \\
0 & \mathbf{H}_{0}
\end{array}\right)+\delta\left(\operatorname{Re}\left(i \mathbf{S}_{1} N_{m}\right)+\left(\begin{array}{cc}
\mathbf{H}_{1} & \mathbf{H}_{2} \\
\mathbf{H}_{2}^{*} & 0
\end{array}\right)\right) \\
& +\delta^{2} \operatorname{Re}\left(i \mathbf{S}_{2} N_{m}+i \mathbf{S}_{1} B_{1}+\mathbf{S}_{0} \beta\right)+O\left(\delta^{3}\right),
\end{aligned}
$$


where $\mathbf{H}_{1} \in \mathcal{H}_{\mu+\mu_{0}}$ has purely imaginary coefficients. Let us first choose $\mathbf{S}_{1}^{\sharp} \in \mathcal{S}_{m}$ such that

$$
\operatorname{Re}\left(i \mathbf{S}_{1}^{\sharp} N_{m}\right)+\left(\begin{array}{cc}
\mathbf{H}_{1} & 0 \\
0 & 0
\end{array}\right)=0
$$

We choose $\mathbf{S}_{1}:=\mathbf{S}_{1}^{\sharp}+\mathbf{S}_{1}^{b}$ where $\mathbf{S}_{1}^{b} \in \mathcal{H}_{m}$ still needs to be determined. Using (6.43) in (6.42) eliminates the upper left block $\mathbf{H}_{1}$ in the coefficient of $\delta$. Moreover, the matrix $\mathbf{H}_{2} \in \mathcal{M}_{\mu+\mu_{0}, \mu-\mu_{0}}(\mathbb{C})$ only depends on $\mathbf{S}_{0}$ which has already been fixed. We can thus apply Young's inequality in (6.42). For appropriate positive constants $c_{0}$ and $C_{0}$, we obtain

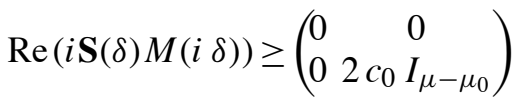

$$
\begin{aligned}
& +\delta \operatorname{Re}\left(i \mathbf{S}_{1}^{b} N_{m}\right)+\delta^{2}\left(\operatorname{Re}\left(i \mathbf{S}_{2} N_{m}+i \mathbf{S}_{1}^{b} B_{1}\right)-C_{0} I\right) \\
& +O\left(\delta^{3}\right) \text {. }
\end{aligned}
$$

At this point, it would seem natural to seek $\mathbf{S}_{1}^{b}$ such that only the lower right $(\mu-$ $\left.\mu_{0}\right) \times\left(\mu-\mu_{0}\right)$ block of $\operatorname{Re}\left(i \mathbf{S}_{1}^{b} N_{m}\right)$ is non-zero. However, this would lead to a disaster since with such a matrix $\mathbf{S}_{1}^{b}$, the upper left coefficient of $\operatorname{Re}\left(i \mathbf{S}_{1}^{b} B_{1}\right)$ would be zero (this is because, opposite to case $\mathrm{I}, b_{m}^{\prime}(0)$ is now a real number). There would be no way to make the coefficient of $\delta^{2}$ positive definite.

The following choice turns out to work:

$$
\mathbf{S}_{1}^{b}:=\left(\begin{array}{cccc}
0 & \ldots & 0 & -i b_{m}^{\prime}(0) \mathfrak{s} \\
0 & \ldots & 0 & 0 \\
\vdots & & & \vdots \\
0 & 0 & & \\
i b_{m}^{\prime}(0) \mathfrak{s} & 0 & \ldots & 0
\end{array}\right), \quad \mathfrak{s} \in \mathbb{R}
$$

This may seem desperate at first glance because $\mathfrak{s}$ should be so large that the upper left coefficient of $\delta^{2}$ in (6.44) is positive. However, choosing $\mathfrak{s}$ large will introduce a large $O(\delta)$ cross term in (6.44). Let us show in detail why the choice (6.45) is appropriate. The upper left coefficient of $\operatorname{Re}\left(i \mathbf{S}_{1}^{b} B_{1}\right)$ is $b_{m}^{\prime}(0)^{2} \mathfrak{s}$. We thus choose $\mathfrak{s}$ large enough such that the upper left coefficient of the matrix

$$
\operatorname{Re}\left(i \mathbf{S}_{1}^{b} B_{1}\right)-C_{0} I
$$

equals 1 . With this choice of $\mathbf{S}_{1}^{b}$, we compute

$$
\left|W^{*} \operatorname{Re}\left(i \mathbf{S}_{1}^{b} N_{m}\right) W\right|=\left|b_{m}^{\prime}(0) \mathfrak{s} \operatorname{Re}\left(W_{2} \bar{W}_{m}\right)\right| \leq c_{0}\left|W_{m}\right|^{2}+C_{1}\left|W_{2}\right|^{2},
$$


where $c_{0}>0$ is the constant that appears in (6.44). Using the latter inequality in (6.44) and assuming that $|\delta|$ is not larger than 1 , we get

$$
\begin{aligned}
\operatorname{Re} & (i \mathbf{S}(\delta) M(i \delta)) \geq\left(\begin{array}{cc}
0 & 0 \\
0 & c_{0} I_{\mu-\mu_{0}}
\end{array}\right) \\
& +\delta^{2}\left(\operatorname{Re}\left(i \mathbf{S}_{2} N_{m}\right)+\operatorname{Re}\left(i \mathbf{S}_{1}^{b} B_{1}\right)-C_{0} I-C_{1}\left(\begin{array}{cc}
0 & 0 \\
0 & I_{m-1}
\end{array}\right)\right) \\
& +O\left(\delta^{3}\right) .
\end{aligned}
$$

Corollary 5.5 shows that we can find a matrix $\mathbf{S}_{2} \in \mathcal{H}_{m}$ such that the coefficient of $\delta^{2}$ in (6.46) is positive definite. Choosing $\delta$ small, we thus end up with the estimate

$$
\operatorname{Re}(i \mathbf{S}(\delta) M(i \delta)) \geq c\left(\begin{array}{cc}
\delta^{2} I_{\mu+\mu_{0}} & 0 \\
0 & I_{\mu-\mu_{0}}
\end{array}\right) .
$$

The symmetrizer $\mathbf{S}(\delta)$ satisfies properties i), ii), iii) of Lemma 5.7 so Theorem 4.4 holds.

The analysis of case IId with $q_{0} \geq 3$ is somehow simpler. The symmetrizer $\mathbf{S}(\delta)$ is chosen of the form

$$
\mathbf{S}(\delta):=\sum_{q=0}^{q_{0}+1} \delta^{q} \mathbf{S}_{q}, \quad \mathbf{S}_{0}, \ldots, \mathbf{S}_{q_{0}-2} \in \mathcal{S}_{m}, \quad \mathbf{S}_{q_{0}-1}, \mathbf{S}_{q_{0}}, \mathbf{S}_{q_{0}+1} \in \mathcal{H}_{m}
$$

The matrix $\mathbf{S}_{0}$ is fixed by applying Lemma 6.3 . We then choose $\mathbf{S}_{0}, \ldots, \mathbf{S}_{q_{0}-2}$ by applying Lemma 6.2. The construction of the matrices $\mathbf{S}_{q_{0}-1}, \mathbf{S}_{q_{0}}, \mathbf{S}_{q_{0}+1}$ follows the arguments that we have just developed above for case IId with $q_{0}=1$. We leave the details to the reader. The final estimate is

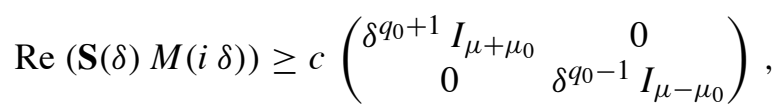

which yields the conclusion of Theorem 4.4.

\subsection{Some remarks}

The symmetrizer construction is probably better understood if, for a fixed even integer $m$, one starts with $m_{0}=m$ and increases $m_{0}$. The situation $m_{0}=m$ corresponds to case I treated in Section 5. The final estimate is

$$
\operatorname{Re}(\mathbf{S}(\delta) M(i \delta)) \geq c\left(\begin{array}{cc}
\delta^{2} I_{\mu} & 0 \\
0 & I_{\mu}
\end{array}\right), \quad c>0, \quad|\delta| \ll 1 .
$$

Each time $m_{0}$ increases of 2 with $m<m_{0} \leq 2 m$, the final estimate is weakened as follows: a coefficient 1 on the diagonal is replaced by a $\delta^{2}$, starting with the 
$(\mu+1)$-th coefficient and ending with the $m$-th coefficient. Since $\delta$ is small, the estimate is weaker. When $m_{0}$ reaches $m_{0}=2 \mathrm{~m}$, that is when $q_{0}=2$ and $\mu_{0}=0$, the diagonal is filled only with $\delta^{2}$. Then we increase $m_{0}$ with $2 m<m_{0} \leq 3 m$. Each time $m_{0}$ increases of 2 , one $\delta^{2}$ coefficient on the diagonal is replaced by a $\delta^{4}$. The process goes on and on. We conjecture that such estimates are optimal.

Compared to case IV below which was already treated by Kreiss [9], dissipation helps! More precisely, the matrix $\operatorname{Re}(\mathbf{S}(\delta) M(i \delta))$ is positive definite for $\delta \neq 0$ in cases I and II. At the opposite, Kreiss' construction in case IV yields a symmetrizer that satisfies $\operatorname{Re}(\mathbf{S}(\delta) M(i \delta))=0$. Here, the higher the dissipation is, the weaker the estimate will be.

Eventually, we observe that in all cases I, IIa,.., IId, the symmetrizer $\mathbf{S}(\delta)$ is a Hermitian matrix and not a real symmetric matrix (except possibly at the origin). Our construction thus differs from Kreiss' choice. In Appendix B we determine necessary conditions for the application of Kreiss' choice. In particular, we show that Kreiss' choice can not work for cases I, II and III. We also observe that as $m_{0}$ gets larger and larger, our symmetrizer $\mathbf{S}(\delta)$ tends more and more to become a symmetric matrix and thus to mimic Kreiss' choice.

\section{Construction of a symmetrizer: case III}

In this section, we consider a matrix $M(\tau)$ of the form (5.1) where $m \geq 3$ is an odd number. The dissipation index $m_{0}$ defined in Proposition 4.5 is even so it is necessarily larger than $m$. We construct in Section 9 a numerical scheme which gives rise to a block of size 3 with a dissipation index equal to 4 . The Euclidean division of $m_{0}$ by $m$ reads

$$
m_{0}=q_{0} m+v_{0}, \quad q_{0} \geq 1, \quad 0 \leq v_{0} \leq m-1 .
$$

The integers $q_{0}$ and $v_{0}$ are simultaneously even or odd. The analogue of Proposition 6.1 is the following result whose proof is omitted. The arguments used to prove Proposition 6.1 work exactly in the same way.

Proposition 7.1. Let $M$ satisfy the assumptions of Theorem 4.4 and correspond to case III. Let the dissipation index $m_{0}$ satisfy (7.1). Then the following properties hold:

- $b_{m}^{\prime}(0) \in \mathbb{R} \backslash\{0\}$,

- for all $q=1, \ldots, q_{0}-1$ and for all $j=1, \ldots, m, i^{q-1} b_{j}^{(q)}(0) \in \mathbb{R}$,

- for all $j=0, \ldots, v_{0}-1, i^{q_{0}-1} b_{m-j}^{\left(q_{0}\right)}(0) \in \mathbb{R}$,

- if $q_{0}$ is even, $b_{m}^{\prime}(0) \operatorname{Im}\left(i^{q_{0}-1} b_{m-v_{0}}^{\left(q_{0}\right)}(0)\right)<0$,

- if $q_{0}$ is odd, $\operatorname{Im}\left(i^{q_{0}-1} b_{m-v_{0}}^{\left(q_{0}\right)}(0)\right)<0$.

Our aim is to construct a symmetrizer $\mathbf{S}(\delta) \in \mathcal{H}_{m}$ that satisfies the properties i), ii), iii) of Lemma 5.7. As for case II, the construction of $\mathbf{S}(\delta)$ depends on the integers 
$q_{0}, v_{0}$ in the Euclidean division (7.1). We shall consider the following three possible cases.

- Case IIIa: $v_{0}=0, q_{0}$ is even.

- Case IIIb: $1 \leq v_{0} \leq m-1, q_{0}$ is even. (Hence $v_{0}$ is also even.)

- Case IIIc: $1 \leq v_{0} \leq m-1, q_{0}$ is odd. (Hence $v_{0}$ is also odd.)

We recall that the integer $\mu$ is given by (4.5). In particular, $\mu$ depends on the sign of $b_{m}^{\prime}(0)$.

\subsection{Case IIIa}

The construction of the symmetrizer follows very closely the analysis in case IIa. We use the expansion (6.8) for $M$ and choose $\mathbf{S}$ of the form (6.10). The matrix $\mathbf{S}_{0}$ is chosen by applying the following analogue of Lemma 6.3.

Lemma 7.2 ([9]). Let $c>0$ and let $C>0$. If $m$ is odd, there exists a matrix $\mathbf{S}_{0} \in \mathcal{S}_{m}$ of the form (5.7) that satisfies

$$
\forall W \in \mathbb{C}^{m}, \quad W^{*} \mathbf{S}_{0} W \geq C\left|W^{u}\right|^{2}-c\left|W^{s}\right|^{2} .
$$

Moreover, the coefficient $s_{1}$ satisfies $s_{1} b_{m}^{\prime}(0)>0$.

The construction of $\mathbf{S}_{0}$ in Lemma 7.2 depends on the sign of $b_{m}^{\prime}(0)$ because the size of $W^{s}$ and $W^{u}$ depend on $b_{m}^{\prime}(0)$. However, the upper left coefficient of $\operatorname{Re}\left(\mathbf{S}_{0} M^{\prime}(0)\right)$ is always positive.

For $K \geq 1$, we fix $\mathbf{S}_{0}$ by applying Lemma 7.2 with $c:=1 / 2$ and $C:=$ $K^{2}+1 / 2$. Then the construction of the matrices $\mathbf{S}_{1}, \ldots, \mathbf{S}_{q_{0}}$ follows by applying exactly the same arguments as in case IIa. Indeed Proposition 7.1 shows that $B_{1}, \ldots, B_{q_{0}-1}$ have real coefficients and the lower left coefficient of $B_{q_{0}}$ satisfies $b_{m}^{\prime}(0) \operatorname{Im}\left(i^{q_{0}-1} b_{m}^{\left(q_{0}\right)}(0)\right)<0$ as in case IIa. The final estimate reads

$$
\operatorname{Re}(\mathbf{S}(\delta) M(i \delta)) \geq c \delta^{q_{0}} I
$$

which yields the conclusion of Theorem 4.4.

\subsection{Case IIIb}

For the sake of clarity, we assume that $b_{m}^{\prime}(0)$ is positive. The relation (4.5) shows that $m$ equals $2 \mu+1$. Since $q_{0}$ is even, we can write $\nu_{0}=2 \mu_{0}$ with $1 \leq \mu_{0} \leq \mu$. We follow the analysis of case IIc, and choose the symmetrizer $\mathbf{S}$ of the form (6.27). We also use the expansion (6.25) of $M(i \delta)$ and recall that the relations (6.26) hold, see Proposition 7.1.

Given $K \geq 1$, the matrix $\mathbf{S}_{0}$ is fixed by applying Lemma 7.2. Then the matrices $\mathbf{S}_{1}, \ldots, \mathbf{S}_{q_{0}-1}$ are chosen in $\mathcal{S}_{m}$ such that the powers $\delta, \ldots, \delta^{q_{0}-1}$ in (6.28) vanish (use Lemma 6.2). The construction of $\mathbf{S}_{q_{0}}$ follows from Lemma 6.7. There is a 
slight subtlety here. The proof of Lemma 6.7 was made for the case $m \equiv 0(2)$. Here $m$ is odd and Lemma 6.5 shows that the image of $\Phi$ is characterized by the relations (6.22), (6.23). When proving Lemma 6.7 for $m$ odd, we need to adapt the definition (6.35) of the matrix $\widetilde{H}_{2}$ by adding one more coefficient on the diagonal. Anyway, this modification is harmless and the conclusion of Lemma 6.7 still holds.

Eventually, the construction of $\mathbf{S}_{q_{0}+1}, \mathbf{S}_{q_{0}+2}$ follows from the same arguments as in case IIc. The final estimate satisfied by $\operatorname{Re}(\mathbf{S}(\delta) M(i \delta))$ is identical to case IIc. We skip the details.

When $b_{m}^{\prime}(0)$ is negative, the analysis of case IIIb follows the same lines. Lemma 6.7 still holds, provided the modification indicated above, because $b_{m}^{\prime}(0)$ and the coefficient $s_{1}$ in the matrix $\mathbf{S}_{0}$ have the same sign (in particular (6.32) holds again and the rest of the proof follows).

\subsection{Case IIIc}

It remains to consider the case when $m$ is odd, $q_{0}$ is odd and therefore $\nu_{0}$ is also odd. For the sake of clarity, we shall assume $b_{m}^{\prime}(0)>0$, so $m=2 \mu+1$. The integer $v_{0}$ is written as $v_{0}:=2 \mu_{0}+1$ with $0 \leq \mu_{0} \leq \mu-1$.

First of all we consider the case $q_{0}=1$. Let $K \geq 1$. We use the expansion (6.39) for $M(i \delta)$ and choose the symmetrizer $\mathbf{S}$ of the form (6.40). The matrix $\mathbf{S}_{0}$ is fixed by applying the analogue of Lemma 6.8.

Lemma 7.3. There exists a matrix $\mathbf{S}_{0} \in \mathcal{H}_{m}$ that satisfies

- for all $W \in \mathbb{C}^{m}, W^{*} \mathbf{S}_{0} W \geq\left(K^{2}+1 / 2\right)\left|W^{u}\right|^{2}-\left|W^{s}\right|^{2} / 2$,

- the upper left coefficient of $\operatorname{Re}\left(\mathbf{S}_{0} M^{\prime}(0)\right)$ is positive,

- the matrix $\operatorname{Re}\left(i \mathbf{S}_{0} N_{m}\right)$ has the form

$$
\left(\begin{array}{cc}
0 & 0 \\
0 & \mathbf{H}_{\mathbf{0}}
\end{array}\right), \quad \mathbf{H}_{\mathbf{0}} \in \mathcal{H}_{\mu-\mu_{0}},
$$

where $\mathbf{H}_{\mathbf{0}}$ is positive definite,

- the upper left $\left(\mu+1+\mu_{0}\right) \times\left(\mu+1+\mu_{0}\right)$ block of $\operatorname{Re}\left(i \mathbf{S}_{0} B_{1}\right)$ has purely imaginary coefficients.

Proof of Lemma 7.3. We indicate the form of the matrix $\mathbf{S}_{0}$ :

$$
\mathbf{S}_{0}:=\left(\begin{array}{cc}
0 & \mathbf{C}_{0} \\
\mathbf{C}_{0}^{*} & \mathbf{D}_{\mathbf{0}}
\end{array}\right)
$$

where the matrices $\mathbf{C}_{0} \in \mathcal{M}_{\mu, \mu+1}(\mathbb{C})$ and $\mathbf{D}_{0} \in \mathcal{H}_{\mu+1}$ are defined as follows:

$$
\mathbf{C}_{0}:=\left(\begin{array}{ccccc}
0 & 0 & \cdots & 0 & c_{1} \\
\vdots & \vdots & 0 & c_{1} & c_{2} \\
0 & 0 & c_{1} & c_{2} & \vdots \\
0 & c_{1} & c_{2} & \cdots & c_{\mu}
\end{array}\right), \quad \mathbf{D}_{0}:=\left(\begin{array}{cccccc}
c_{1} & \ldots & c_{\mu_{0}+1} & c_{\mu_{0}+2} & \ldots & c_{\mu+1} \\
\ddots & \vdots & \vdots & & \vdots \\
& c_{2} \mu_{0}+1 & c_{2} \mu_{0}+1 & \cdots & c_{\mu+\mu_{0}+1} \\
& & & & \widetilde{\mathbf{D}}_{0} & \\
\star & & & & &
\end{array}\right) .
$$


The idea is to choose first $c_{2}=\cdots=c_{2} \mu_{0}=0$. Then we choose inductively $c_{1}, \ldots, c_{2} \mu_{0+1}>0$ sufficiently large. Then we choose $c_{2} \mu_{0}+2, \ldots, c_{\mu+\mu_{0}+1} \in \mathbb{C}$ so that the upper left $\left(\mu+1+\mu_{0}\right) \times\left(\mu+1+\mu_{0}\right)$ block of $\operatorname{Re}\left(i \mathbf{S}_{0} B_{1}\right)$ has purely imaginary coefficients. Eventually, we choose $\widetilde{\mathbf{D}}_{0} \in \mathcal{H}_{\mu-\mu_{0}}$ positive definite large enough in such a way that the block $\mathbf{H}_{0}$ is positive definite (the existence of $\widetilde{\mathbf{D}}_{0}$ is ensured by Lemma 5.4). The details are similar to the proof of Lemma 6.8.

Once $\mathbf{S}_{0}$ is fixed, the construction of $\mathbf{S}_{1}$ and $\mathbf{S}_{2}$ follows the method used in case IId. This completes the proof of Theorem 4.4 in case III.

\section{Construction of a symmetrizer: case IV}

First of all, we make the assumption on the dissipation index more explicit.

Proposition 8.1. Let $M$ satisfy the assumptions of Theorem 4.4 and correspond to case IV. Then $b_{m}^{\prime}(0)$ is a non-zero real number, and there holds

$$
\forall q \geq 1, \quad \forall j=1, \ldots, m, \quad i^{q-1} b_{j}^{(q)}(0) \in \mathbb{R} .
$$

In particular $M(i \delta)$ has purely imaginary coefficients when $\delta \in \mathbb{R}$ is sufficiently small.

The method used in the proof of Proposition 6.1 applies and the process has no end (because all the derivatives $i^{\nu-1} \ell^{(v)}(0)$ are real). To show that $M(i \delta)$ has purely imaginary coefficients, one expands $M(i \delta)$ in power series. This is possible because the functions $b_{j}$ are holomorphic.

Since $M(i \delta)$ has purely imaginary coefficients for real $\delta$, Kreiss' construction in [9] applies.

Theorem 8.2 ( [9]). For all $K \geq 1$, there exists an open interval $I_{K}$ that contains 0 and there exists a $\mathcal{C}^{\infty}$ function $\mathbf{S}$ defined on $I_{K}$ with values in $\mathcal{S}_{m}$ that satisfies

- for all $\delta \in I_{K}, \operatorname{Re}(\mathbf{S}(\delta) M(i \delta))=0$,

- for all $W \in \mathbb{C}^{m}, W^{*} \mathbf{S}(0) W \geq\left(K^{2}+1 / 2\right)\left|W^{u}\right|^{2}-\left|W^{s}\right|^{2} / 2$,

- the upper left coefficient of $\operatorname{Re}\left(\mathbf{S}(0) M^{\prime}(0)\right)$ is positive.

In particular, Lemma 5.7 shows that the conclusion of Theorem 4.4 holds. We have therefore proved Theorem 4.4 in all possible cases.

We refer to Appendix B for a converse result of Theorem 8.2.

\section{Examples of dissipative blocks}

\subsection{An example of large size block for case I}

We are going to show on an example that blocks of the fourth type and of arbitrarily large size can occur in the discrete block structure. In the example below, such 
blocks correspond to case I. We consider the scalar transport equation

$$
\partial_{t} u+\partial_{x} u=0 .
$$

We recall that $\mathcal{A}(\kappa)$ denotes the symbol defined by (2.4). For scalar equations and one-step schemes $(s=0), \mathcal{A}(\kappa)$ is a complex number so the uniform powerboundedness and geometric regularity of eigenvalues reduce to the inequality $|\mathcal{A}(\kappa)| \leq 1$ for all $\kappa \in \mathbb{S}^{1}$ (the well-known Von Neumann condition).

Let us consider an integer $J \in \mathbb{N}$ that is fixed once and for all. Then we define the numbers

$$
\forall j=0, \ldots, J, \quad q_{j}:=\frac{1}{2^{2 J+1}}\left(\begin{array}{c}
2 J+1 \\
J-j
\end{array}\right) \frac{1}{2 j+1} .
$$

Using these numbers, we define the following finite difference operator (the operator is an approximation of the space derivative $\partial_{x}$, as shown below):

$$
Q:=\sum_{j=0}^{J} q_{j}\left(T^{1+2 j}-T^{-1-2 j}\right) .
$$

Following [6, Chapter 6], we consider the Runge-Kutta scheme of order 3 that is obtained after using the operator $Q$ for the spatial discretization ( $\lambda$ still denotes the Courant number $\Delta t / \Delta x$ )

$$
U_{j}^{n+1}=\sum_{\ell=0}^{3} \frac{(-\lambda Q)^{\ell}}{\ell !} U_{j}^{n}
$$

We compute

$$
\mathcal{A}(\kappa)=\sum_{\ell=0}^{3} \frac{(-\lambda \widehat{Q}(\kappa))^{\ell}}{\ell !}, \quad \widehat{Q}(\kappa)=\sum_{j=0}^{J} q_{j}\left(\kappa^{1+2 j}-\kappa^{-1-2 j}\right) .
$$

For the scheme (9.3), we have $r=p=3(1+2 J)$ and

$$
\forall z \in \mathbb{C} \backslash\{0\}, \quad \mathbb{A}_{-r}(z)=\frac{\lambda^{3} q_{J}^{3}}{6 z}, \quad \mathbb{A}_{p}(z)=-\mathbb{A}_{-r}(z) .
$$

Assumption 2.2 is thus satisfied. We now check the $\ell^{2}$-stability of the scheme (9.3) and compute

$$
\left|\mathcal{A}\left(\mathrm{e}^{i \xi}\right)\right|^{2}=1-\frac{\lambda^{4}}{12} h(\xi)^{4}\left(1-\frac{\lambda^{2}}{3} h(\xi)^{2}\right), \quad h(\xi):=\sum_{j=0}^{J} 2 q_{j} \sin ((2 j+1) \xi) .
$$

The main properties of the function $h$ are summarized below. 
Lemma 9.1. Let the numbers $q_{j}$ be defined by (9.2) and let $h$ be defined by (9.5). Then $h$ is odd and satisfies

$$
\forall \xi \in \mathbb{R}, \quad h^{\prime}(\xi)=\cos ^{2 J+1} \xi .
$$

The function $h$ vanishes exactly for $\xi \in \mathbb{Z} \pi$. The maximum of $h$ on $\mathbb{R}$, that we denote $\beta_{J}$, is positive and is attained when $\xi \in \pi / 2+\mathbb{Z} 2 \pi$.

The scheme (9.3) is $\ell^{2}$-stable and geometrically regular if and only if $f^{6} \lambda \leq$ $\sqrt{3} / \beta_{J}$. Moreover, the scheme (9.3) is consistent with the transport equation (9.1).

Proof of Lemma 9.1. It is clear that $h$ is odd, and we now differentiate $h$ using the expression (9.2) of the $q_{j}$ 's, obtaining

$$
\begin{aligned}
h^{\prime}(\xi) & =\frac{1}{2^{2 J}} \sum_{j=0}^{J}\left(\begin{array}{c}
2 J+1 \\
J-j
\end{array}\right) \cos ((2 j+1) \xi) \\
& =\frac{1}{2^{2 J}} \sum_{j=0}^{J}\left(\begin{array}{c}
2 J+1 \\
j
\end{array}\right) \cos ((2 J+1-2 j) \xi) \\
& =\frac{1}{2^{2 J+1}} \sum_{j=0}^{2 J+1}\left(\begin{array}{c}
2 J+1 \\
j
\end{array}\right) \cos ((2 J+1-2 j) \xi) \\
& =\operatorname{Re}\left(\frac{\mathrm{e}^{i \xi}+\mathrm{e}^{-i \xi}}{2}\right)^{2 J+1}=\cos ^{2 J+1} \xi,
\end{aligned}
$$

where we have first changed $j$ for $J-j$, and then used the symmetry of the binomial coefficients.

It follows that $h$ behaves exactly as the sine function: $h$ vanishes at 0 , is increasing on $[0, \pi / 2]$, attains its maximum at $\pi / 2$, is decreasing on $[\pi / 2,3 \pi / 2]$ and vanishes at $\pi$, attains its minimum at $3 \pi / 2$, and so on.

We see on the relation (9.5) that $\left|\mathcal{A}\left(\mathrm{e}^{i \xi}\right)\right|$ is bounded by 1 for all $\xi \in \mathbb{R}$ if and only if $\lambda \max _{\mathbb{R}}|h| \leq \sqrt{3}$, which is equivalent to $\lambda \leq \sqrt{3} / \beta_{J}$.

It remains to prove that the scheme (9.3) is consistent with the transport equation (9.1). We have

$$
\mathcal{A}\left(\mathrm{e}^{i \xi}\right)=1-\frac{\lambda^{2}}{2} h(\xi)^{2}-i \lambda h(\xi)\left(1-\frac{\lambda^{2}}{6} h(\xi)^{2}\right) .
$$

Since $h(0)=0$ and $h^{\prime}(0)=1$, we have $h(\xi) \sim \xi$ for small $\xi$, and we obtain

$$
\mathcal{A}\left(\mathrm{e}^{i \xi}\right)=1-i \lambda \xi+O\left(\xi^{2}\right)=\mathrm{e}^{-i \lambda \xi}+O\left(\xi^{2}\right) .
$$

Applying Theorem 5.2.5 in [6], the scheme (9.3) is consistent with (9.1).

6 The value of $\beta_{J}$ is the Wallis integral $\int_{0}^{\pi / 2} \cos ^{2 J+1} \xi \mathrm{d} \xi$, that is $2^{2 J}(J !)^{2} /(2 J+1) !$. Since $\beta_{J}$ tends to 0 as $J$ tends to $+\infty$, the range of stability for the scheme (9.3) is getting larger and larger with $J$ going to $+\infty$. 
We analyze the behavior of $\mathcal{A}(\kappa)$ when it touches the unit circle $\mathbb{S}^{1}$. We assume that the CFL condition is chosen in an optimal way, that is $\lambda=\sqrt{3} / \beta_{J}$. According to (9.5) and to Lemma 9.1, we have $\mathcal{A}(\kappa) \in \mathbb{S}^{1}$ if and only if $\kappa \in\{ \pm 1, \pm i\}$. More precisely, we have $\mathcal{A}( \pm 1)=1$, and $\mathcal{A}( \pm i)=-1 / 2 \mp i \sqrt{3} / 2$.

Differentiating $\mathcal{A}\left(\mathrm{e}^{i \xi}\right)$ with respect to $\xi$ and using the properties of $h$, we obtain $\mathcal{A}^{\prime}( \pm 1)=\mp \lambda \neq 0$. The point 1 which is attained for $\xi \in \mathbb{Z} \pi$ on the parametrized curve $\left\{\mathcal{A}\left(\mathrm{e}^{i \xi}\right), \xi \in \mathbb{R}\right\}$ is a regular point, see Figure 9.1.

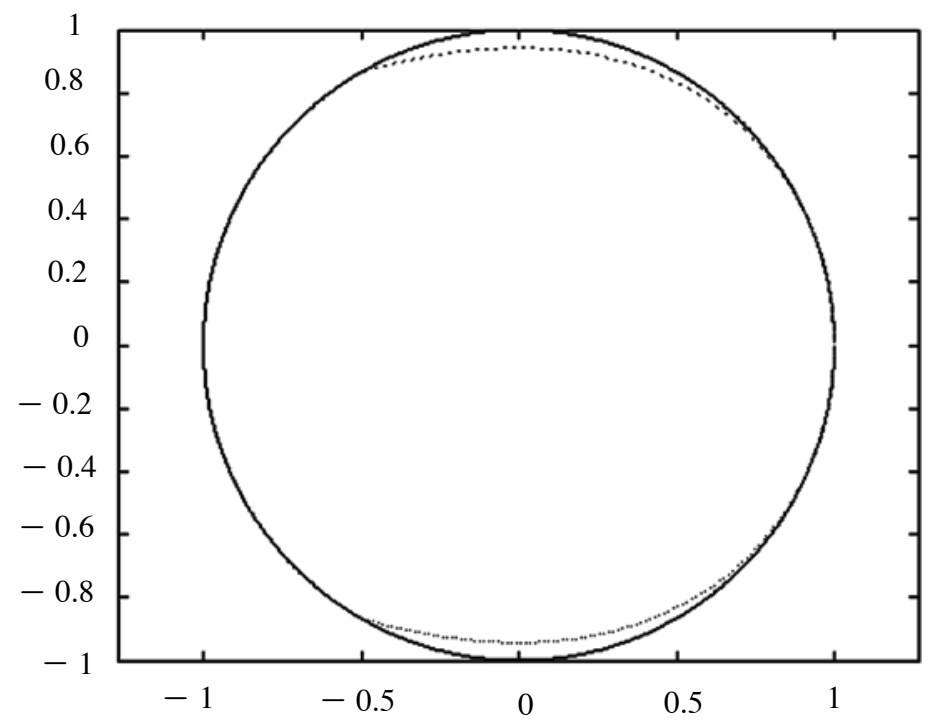

Figure 9.1. The parametrized curve $\left\{\mathcal{A}\left(\mathrm{e}^{i \xi}\right), \xi \in \mathbb{R}\right\}$ for $J=1$ in dots, with $\mathcal{A}\left(\mathrm{e}^{i \xi}\right)$ given by (9.4). The unit circle is plotted in black line.

Let us now study the function $\mathcal{A}\left(\mathrm{e}^{i \xi}\right)$ in the neighborhood of $\xi=\pi / 2$. Using Lemma 9.1, we have $h^{\prime}(\pi / 2)=\cdots=h^{(2 J+1)}(\pi / 2)=0$, and $h^{(2 J+2)}(\pi / 2)=$ $-(2 J+1)$ !. Performing a Taylor expansion, we obtain

$$
\mathcal{A}\left(\mathrm{e}^{i \xi}\right)=-\frac{1}{2}-i \frac{\sqrt{3}}{2}+\frac{\lambda(\sqrt{3}-i / 2)}{2 J+2}(\xi-\pi / 2)^{2 J+2}+O(\xi-\pi / 2)^{2 J+3} .
$$

This expansion has two consequences. First, we obtain $\mathcal{A}^{\prime}(i)=\ldots=\mathcal{A}^{(2 J+1)}(i)=$ $0, \mathcal{A}^{(2 J+2)}(i) \neq 0$. The points $-1 / 2 \pm i \sqrt{3} / 2$ on the curve $\left\{\mathcal{A}\left(\mathrm{e}^{i \xi}\right), \xi \in \mathbb{R}\right\}$ are singular points, see Figure 9.1. In the block reduction of $\mathbb{M}\left(\mathrm{e}^{ \pm i 2 \pi / 3}\right)$, the existence of such singular points gives rise to blocks of the fourth type and of size $2 J+2$, see [4, Section 3]. Moreover, we also obtain

$$
\left|\mathcal{A}\left(\mathrm{e}^{i \xi}\right)\right|^{2}=1-\frac{\lambda \sqrt{3}}{4(J+1)}(\xi-\pi / 2)^{2 J+2}+O(\xi-\pi / 2)^{2 J+3},
$$

which corresponds to a dissipation of order $2 J+2$. 
When the CFL condition is satisfied in an optimal way, the scheme (9.3) gives an example of a block of the fourth type of size $2 J+2$ with a dissipation index $m_{0}=2 J+2$. The symmetrizer construction for this block corresponds to case I.

It does not seem so obvious to find a one-step scheme $(s=0)$ that produces a block of size $m \geq 2$ for which the dissipation index is larger than $m$ (case II or case III). In the following paragraph, we construct a two-step scheme $(s=1)$ that produces a block of size 3 and that corresponds to case III.

\subsection{An example of block for case III}

We still consider the transport equation (9.1). The space derivative is discretized first by means of a finite difference operator, leading to the ordinary differential equation

$$
\dot{U}_{j}=\frac{1}{\Delta x} Q U_{j}, \quad j \in \mathbb{Z} .
$$

Then we apply the Adams-Bashforth quadrature rule of order 2. The numerical scheme thus reads

$$
U_{j}^{n+1}=U_{j}^{n}+\lambda\left(\frac{3}{2} Q U_{j}^{n}-\frac{1}{2} Q U_{j}^{n-1}\right) .
$$

We choose the finite difference operator $Q$ of the form

$$
Q=\sum_{\ell=-3}^{4} q_{\ell} T^{\ell}
$$

where the real numbers $q_{-3}, \ldots, q_{4}$ are defined as the solution to the linear system

$$
\left(\begin{array}{cccccccc}
1 & 1 & 1 & 1 & 1 & 1 & 1 & 1 \\
-3 & -2 & -1 & 0 & 1 & 2 & 3 & 4 \\
9 & 4 & 1 & 0 & 1 & 4 & 9 & 16 \\
-1 & 1 & -1 & 1 & -1 & 1 & -1 & 1 \\
3 & -2 & 1 & 0 & -1 & 2 & -3 & 4 \\
-9 & 4 & -1 & 0 & -1 & 4 & -9 & 16 \\
27 & -8 & 1 & 0 & -1 & 8 & -27 & 64 \\
-81 & 16 & -1 & 0 & -1 & 16 & -81 & 256
\end{array}\right)\left(\begin{array}{c}
q-3 \\
q-2 \\
q_{-1} \\
q_{0} \\
q_{1} \\
q_{2} \\
q_{3} \\
q_{4}
\end{array}\right)=\left(\begin{array}{c}
0 \\
-1 \\
1 \\
-1 \\
0 \\
0 \\
-1 \\
1
\end{array}\right) .
$$

The reader can easily check on a computer that the matrix of the above linear system is invertible so the scheme (9.6) is well-defined. In this case, we have $s=1, r=3$, $p=4$, and

$$
\forall z \in \mathbb{C} \backslash\{0\}, \quad \mathbb{A}_{-r}(z)=\frac{\lambda q-3}{2 z}\left(\frac{1}{z}-3\right), \quad \mathbb{A}_{p}(z)=\frac{\lambda q_{4}}{2 z}\left(\frac{1}{z}-3\right) .
$$

It follows from (9.7) that $q_{-3}$ and $q_{4}$ are non-zero so Assumption 2.2 is satisfied. We now try to verify the assumptions of Theorem $2.3: \ell^{2}$-stability and geometric regularity of eigenvalues. The proof of the following Lemma is a straightforward verification and is thus omitted. 
Lemma 9.2. Let the $q_{\ell}$ 's be defined by (9.7). Then the function

$$
\xi \in \mathbb{R} \longmapsto q(\xi):=\sum_{\ell=-3}^{4} q_{\ell} \mathrm{e}^{i \ell \xi},
$$

satisfies

$$
\begin{aligned}
& q(0)=0, \quad q^{\prime}(0)=-i, \quad q^{\prime \prime}(0)=-1, \\
& q(\pi)=-1, \quad q^{\prime}(\pi)=q^{\prime \prime}(\pi)=0, \quad q^{(3)}(\pi)=i, \quad q^{(4)}(\pi)=1 .
\end{aligned}
$$

In particular, the scheme (9.6) is consistent with the transport equation (9.1).

The amplification matrix $\mathcal{A}\left(\mathrm{e}^{i \xi}\right)$ associated with the scheme (9.6) is

$$
\mathcal{A}\left(\mathrm{e}^{i \xi}\right)=\left(\begin{array}{c}
1+\frac{3 \lambda}{2} q(\xi)-\frac{\lambda}{2} q(\xi) \\
1
\end{array}\right) .
$$

We wish to determine the CFL parameters $\lambda$ for which $\mathcal{A}$ satisfies the assumptions of Theorem 2.3. More precisely, we are going to show that for $\lambda \in] 0,1]$, the amplification matrix $\mathcal{A}\left(\mathrm{e}^{i \xi}\right)$ satisfies the stability and geometric regularity conditions of Theorem 2.3.

We shall need the following result.

\section{Lemma 9.3. The mapping}

$$
\kappa \in \mathbb{S}^{1} \longmapsto \frac{2 \kappa(\kappa-1)}{3 \kappa-1},
$$

is injective and thus defines a closed simple curve $\mathcal{C} \subset \mathbb{C} \simeq \mathbb{R}^{2}$. The interior $\mathcal{I}$ of $\mathcal{C}$ is a strictly convex region.

Proof of Lemma 9.3. We consider the mapping

$$
\theta \in[-\pi, \pi] \longmapsto \frac{2 \mathrm{e}^{i \theta}\left(\mathrm{e}^{i \theta}-1\right)}{3 \mathrm{e}^{i \theta}-1}=x(\theta)+i y(\theta) .
$$

Direct computations yield $y(0)=y( \pm \pi)=0$, and $\pm y(\theta)>0$ if $\pm \theta \in] 0, \pi[$. Furthermore, $x$ is increasing on $[-\pi, 0]$ and decreasing on $[0, \pi]$. These properties imply that $\mathcal{C}$ is a simple closed curve (see Figure 9.2 for a representation of $\mathcal{C}$ ). The reader can also check that $\left(x^{\prime}\right)^{2}+\left(y^{\prime}\right)^{2}$ does not vanish so every point of $\mathcal{C}$ is regular.

The interior of $\mathcal{C}$ is well-defined thanks to Jordan's Theorem. It is strictly convex provided that the curvature of $\mathcal{C}$ is nonnegative and vanishes at finitely many points. This amounts to proving that $x^{\prime} y^{\prime \prime}-x^{\prime \prime} y^{\prime}$ is nonnegative and vanishes at finitely many points. We compute

$$
x^{\prime}(\theta) y^{\prime \prime}(\theta)-x^{\prime \prime}(\theta) y^{\prime}(\theta)=\left.\frac{6(1-X)\left(3 X^{2}-3 X+4\right)}{(5-3 X)^{3}}\right|_{X=\cos \theta} \geq 0,
$$

so $\mathcal{I}$ is strictly convex. 


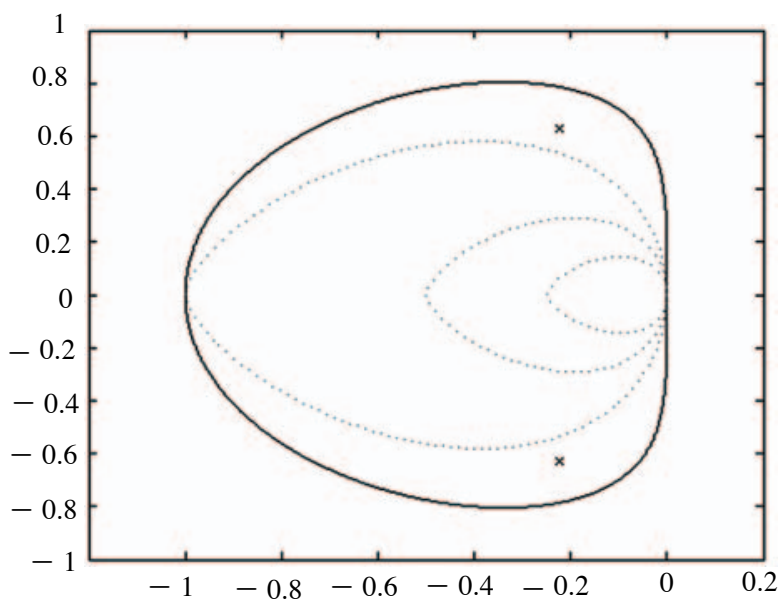

Figure 9.2. The curve $\mathcal{C}$ (black line), and the curve $\{\lambda q(\xi), \xi \in \mathbb{R}\}$ for $\lambda=1 / 4$, $\lambda=1 / 2$ and $\lambda=1$ (dots). The crosses represent the points $-2 / 9 \pm i 4 \sqrt{2} / 9$.

The curve $\mathcal{C}$ is relevant for the following reason: the amplification matrix $\mathcal{A}\left(\mathrm{e}^{i \xi}\right)$ in (9.9) has an eigenvalue on the unit circle $\mathbb{S}^{1}$ if and only if $\lambda q(\xi) \in \mathcal{C}$. More precisely, we have:

- If $\lambda q(\xi) \in \mathcal{I}$, then both eigenvalues of $\mathcal{A}\left(\mathrm{e}^{i \xi}\right)$ belong to $\mathbb{D}$ (examine the case $\lambda q(\xi)=-1 / 2$ and use a connectedness argument).

- If $\lambda q(\xi)$ belongs to the exterior of $\mathcal{C}$, then $\mathcal{A}\left(\mathrm{e}^{i \xi}\right)$ has one eigenvalue in $\mathbb{D}$ and one eigenvalue in $\mathcal{U}$ (examine the case $\lambda q(\xi)=1$ and use a connectedness argument).

- If $\lambda q(\xi) \in \mathcal{C}, \mathcal{A}\left(\mathrm{e}^{i \xi}\right)$ has one eigenvalue on $\mathbb{S}^{1}$ and one eigenvalue in $\mathbb{D}$ (use Lemma 9.3).

Eventually, it is straightforward to check that $\mathcal{A}\left(\mathrm{e}^{i \xi}\right)$ has a double eigenvalue if and only if $\lambda q(\xi)=-2 / 9 \pm i 4 \sqrt{2} / 9$. These two points are marked by crosses on Figure 9.2.

Let us consider the case $\lambda=1$. For $\xi=0$, Lemma 9.2 shows that $\mathcal{A}(1)$ has the eigenvalues 1 and 0 . For $\xi \in \mathbb{R}$ close to 0 , we make a Taylor expansion for the eigenvalue $\omega_{1}(\xi)$ of $\mathcal{A}\left(\mathrm{e}^{i \xi}\right)$ close to 1 . Using Lemma 9.2, we obtain

$$
\omega_{1}^{\prime}(0) \neq 0, \quad\left|\omega_{1}(\xi)\right|^{2}=1-\xi^{2}+o\left(\xi^{2}\right) .
$$

In particular, $q(0) \in \mathcal{C}$ and $q(\xi) \in \mathcal{I}$ for $\xi \neq 0$ sufficiently small.

For $\xi=\pi$, Lemma 9.2 shows that $\mathcal{A}(-1)$ has the eigenvalues -1 and $1 / 2$. For $\xi \in \mathbb{R}$ close to $\pi$, we make another Taylor expansion for the eigenvalue $\omega_{2}(\xi)$ of $\mathcal{A}\left(\mathrm{e}^{i \xi}\right)$ close to -1 . Using Lemma 9.2 again, we obtain $\omega_{2}^{\prime}(\pi)=\omega_{2}^{\prime \prime}(\pi)=0, \quad \omega_{2}^{(3)}(\pi) \neq 0, \quad\left|\omega_{2}(\xi)\right|^{2}=1-\frac{(\xi-\pi)^{4}}{9}+o\left((\xi-\pi)^{4}\right)$. 
In particular, $q(\pi) \in \mathcal{C}$ and $q(\xi) \in \mathcal{I}$ for $\xi-\pi \neq 0$ sufficiently small. In the block reduction for $\mathbb{M}(-1)$, the behavior of $\omega_{2}$ yields a $3 \times 3$ block with a dissipation index equal to 4 . Figure 9.2 gives numerical evidence that for $\xi \notin \mathbb{Z} \pi, q(\xi)$ belongs to $\mathcal{I}$ the interior of $\mathcal{C}$. The Von Neumann condition is thus satisfied for $\lambda=1$. We refer to Figure 9.3 for a representation of the spectrum ${ }^{7}$ of $\mathcal{A}\left(\mathrm{e}^{i \xi}\right)$ when $\lambda$ equals 1 . Since the eigenvalues of $\mathcal{A}( \pm 1)$ are simple, eigenvalues and eigenvectors of $\mathcal{A}(\kappa)$ depend holomorphically on $\kappa$ for $\kappa$ close to \pm 1 . The combination of geometric regularity and of the Von Neumann condition implies that $\mathcal{A}$ satisfies the uniform power boundedness condition of Theorem 2.3.

When $\lambda \in] 0,1$, the strict convexity of $\mathcal{I}$ implies that $\lambda q(\xi)$ belongs to $\mathcal{I}$ for $\xi \notin \mathbb{Z} 2 \pi$. Hence the eigenvalues of $\mathcal{A}\left(\mathrm{e}^{i \xi}\right)$ belong to $\mathbb{D}$. For $\xi=0$, we have $\lambda q(\xi)=0$ and $\mathcal{A}(1)$ has the eigenvalues 1 and 0 . A Taylor expansion gives

$$
\omega_{1}^{\prime}(0) \neq 0, \quad\left|\omega_{1}(\xi)\right|^{2}=1-\lambda \xi^{2}+o\left(\xi^{2}\right) .
$$

The Von Neumann condition is satisfied as well as the geometric regularity condition. Up to admitting that $q(\xi)$ belongs to $\mathcal{I}$ for $\xi \notin \mathbb{Z} \pi$ (the latter property being clearly verified on Figure 9.2), we have proved that $\mathcal{A}$ satisfies all assumptions of Theorem 2.3 when $\lambda \in] 0,1]$. Furthermore, the case $\lambda=1$ provides with an example of $3 \times 3$ block for case III.

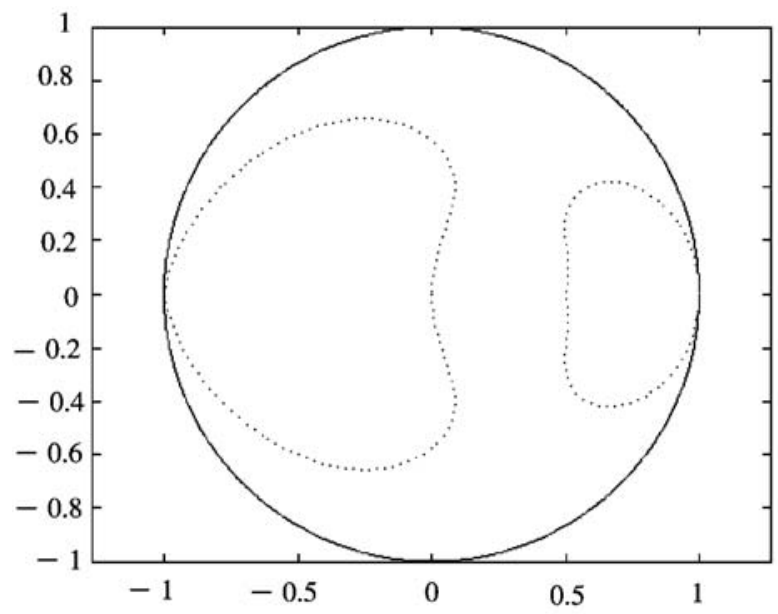

Figure 9.3. The eigenvalues of $\left\{\mathcal{A}(\kappa), \kappa \in \mathbb{S}^{1}\right\}$ for $\lambda=1$ (dots), and the unit circle (black line).

7 Observe that the eigenvalues do not cross because the curve $\{q(\xi), \xi \in \mathbb{R}\}$ does not meet the points $-2 / 9 \pm i 4 \sqrt{2} / 9$. 


\section{A. Some results about matrices}

\section{A.1. Proof of Lemma 5.4}

For the reader's convenience, we recall the statement of Lemma 5.4.

Lemma A.1. Let $C_{1}, C_{2} \in \mathbb{R}$, let $c>0$, and let $v$ be an integer. Then there exists a matrix $H \in \mathcal{H}_{v}$ such that for all $W=\left(W_{1}, \ldots, W_{v}\right) \in \mathbb{C}^{v}$, there holds

$$
\begin{aligned}
& W^{*} H W \geq C_{1}|W|^{2}, \\
& W^{*} \operatorname{Re}\left(i H N_{v}\right) W \geq-c\left|W_{1}\right|^{2}+C_{2} \sum_{j=2}^{v}\left|W_{j}\right|^{2} .
\end{aligned}
$$

Lemma 5.4 is a refined version of Lemma 5.7 in [3, Chapter VII] where it is shown that there exists a Hermitian matrix $H$ satisfying (A.2). Here we want to satisfy (A.1) and (A.2) simultaneously.

Proof of Lemma 5.4. There is nothing to prove if $v=1$ since $N_{1}=0$, and it is therefore sufficient to choose $H=C_{1}$. We thus assume $v \geq 2$ in what follows. We choose the matrix $H$ of the form

$$
H:=\left(\begin{array}{ccc}
a_{1} & & 0 \\
& \ddots & \\
0 & & a_{\nu}
\end{array}\right)-i\left(\begin{array}{cccc}
0 & -g_{1} & & 0 \\
g_{1} & \ddots & \ddots & \\
& \ddots & \ddots & -g_{\nu-1} \\
0 & & g_{\nu-1} & 0
\end{array}\right),
$$

where $a_{1}, \ldots, a_{v}, g_{1}, \ldots, g_{v-1}$ are real numbers to be fixed appropriately. We compute the following relations for all vector $W \in \mathbb{C}^{\nu}$ :

$$
\begin{aligned}
& W^{*} H W=\sum_{j=1}^{\nu} a_{j}\left|W_{j}\right|^{2}-2 \sum_{j=1}^{\nu-1} g_{j} \operatorname{Im}\left(\overline{W_{j}} W_{j+1}\right), \\
& W^{*} \operatorname{Re}\left(i H N_{\nu}\right) W= \sum_{j=2}^{\nu} g_{j-1}\left|W_{j}\right|^{2}-\sum_{j=1}^{\nu-1} a_{j} \operatorname{Im}\left(\overline{W_{j}} W_{j+1}\right) \\
&-\sum_{j=1}^{\nu-2} g_{j} \operatorname{Re}\left(\overline{W_{j}} W_{j+2}\right) .
\end{aligned}
$$

The idea is to choose first $a_{1}$, then $g_{1}$, then $a_{2}$, then $g_{2}$ and so on, and in the end to choose $a_{v}$. More precisely, it follows from an easy induction argument using Young's inequality that for all $J=1, \ldots, v-1$, there exist some real numbers $a_{1}, g_{1}, \ldots, a_{J}, g_{J}$ such that the inequalities

$$
\sum_{j=1}^{J} a_{j}\left|W_{j}\right|^{2}-2 \sum_{j=1}^{J-1} g_{j} \operatorname{Im}\left(\overline{W_{j}} W_{j+1}\right) \geq C_{1} \sum_{j=1}^{J-1}\left|W_{j}\right|^{2}+\left(C_{1}+1\right)\left|W_{J}\right|^{2},
$$


and

$$
\begin{aligned}
\sum_{j=2}^{J+1} g_{j-1}\left|W_{j}\right|^{2}-\sum_{j=1}^{J} & a_{j} \operatorname{Im}\left(\overline{W_{j}} W_{j+1}\right)-\sum_{j=1}^{J-1} g_{j} \operatorname{Re}\left(\overline{W_{j}} W_{j+2}\right) \\
& \geq-c\left|W_{1}\right|^{2}+C_{2} \sum_{j=2}^{J}\left|W_{j}\right|^{2}+\left(C_{2}+1\right)\left|W_{J+1}\right|^{2},
\end{aligned}
$$

hold for all $W \in \mathbb{C}^{v}$.

We can therefore fix some real numbers $a_{1}, g_{1}, \ldots, a_{v-1}, g_{v-1}$ such that (A.5) and (A.6) hold with $J=v-1$. Using (A.6) in (A.4), we already find that the inequality (A.2) is satisfied by $H$. This inequality does not involve the coefficient $a_{\nu}$. If we use (A.5) in (A.3), we obtain

$$
W^{*} H W \geq a_{\nu}\left|W_{\nu}\right|^{2}-2 g_{\nu-1} \operatorname{Im}\left(\overline{W_{\nu-1}} W_{\nu}\right)+\left|W_{\nu-1}\right|^{2}+C_{1} \sum_{j=1}^{\nu-1}\left|W_{j}\right|^{2} .
$$

Applying Young's inequality and choosing $a_{v}$ large enough, we can construct the matrix $H$ such that (A.1) holds. The proof of Lemma 5.4 is now complete.

\section{A.2. Proof of Lemma 6.3}

We first recall the statement of Lemma 6.3.

Lemma A.2 ([9]). Let $a \in \mathbb{R}$. Let $c>0$ and let $C \in \mathbb{R}$. If $m$ is even, there exists $a$ matrix $S \in \mathcal{S}_{m}$ of the form (5.7) with $s_{1}=a$, and that satisfies

$$
\forall W \in \mathbb{C}^{m}, \quad W^{*} S W \geq C\left|W^{u}\right|^{2}-c\left|W^{s}\right|^{2} .
$$

Proof of Lemma 6.3. Consider the case $m=2$. We wish to choose $s_{2} \in \mathbb{R}$ sufficiently large such that the inequality

$$
\left(\overline{W_{1}} \overline{W_{2}}\right)\left(\begin{array}{ll}
0 & a \\
a & s_{2}
\end{array}\right)\left(\begin{array}{l}
W_{1} \\
W_{2}
\end{array}\right) \geq C\left|W_{2}\right|^{2}-c\left|W_{1}\right|^{2}
$$

holds for all $W \in \mathbb{C}^{2}$. The result is clear and is based on Young's inequality.

The proof of Lemma 6.3 in the case $m \geq 4$ follows the same lines. More precisely, we consider a real symmetric matrix $S$ of size $m=2 \mu$ and of the form (5.7). The first coefficient $s_{1}$ is fixed by choosing $s_{1}:=a$. It remains to choose the coefficients $s_{2}, s_{3}, \ldots, s_{2} \mu$ appropriately. We first make the choice $s_{3}=s_{5}=$ $\cdots=s_{2 \mu-1}:=0$. We compute

$$
\begin{aligned}
W^{*} S W= & 2 a \sum_{k=1}^{\mu} \operatorname{Re}\left(\overline{W_{k}} W_{m+1-k}\right)+\sum_{k=1}^{\mu} s_{2 k}\left|W_{\mu+k}\right|^{2} \\
& +\sum_{k=1}^{\mu-1} 2 s_{2 k} \sum_{j=2 k}^{\mu+k-1} \operatorname{Re}\left(\overline{W_{j}} W_{m+2 k-j}\right) .
\end{aligned}
$$


We apply Young's inequality for the first term in the right-hand side. There exists a constant $C_{0}>0$, that only depends on $a$ and $c$ such that

$$
\begin{aligned}
W^{*} S W \geq & -\frac{c}{2^{\mu-1}}\left|W^{s}\right|^{2}+\sum_{k=1}^{\mu}\left(s_{2 k}-C_{0}\right)\left|W_{\mu+k}\right|^{2} \\
& +\sum_{k=1}^{\mu-1} 2 s_{2 k} \sum_{j=2 k}^{\mu+k-1} \operatorname{Re}\left(\overline{W_{j}} W_{m+2 k-j}\right) .
\end{aligned}
$$

The following property is proved by induction on $J=1, \ldots, \mu-1$ : there exist some coefficients $s_{2}, \ldots, s_{2} J>0$ and there exists a constant $C_{J}>0$ that only depend on $a, c, C$ such that the inequality

$$
\begin{aligned}
& \sum_{k=1}^{J}\left(s_{2 k}-C_{0}\right)\left|W_{\mu+k}\right|^{2}+\sum_{k=1}^{J} 2 s_{2 k} \sum_{j=2 k}^{\mu+k-1} \operatorname{Re}\left(\overline{W_{j}} W_{m+2 k-j}\right) \\
& \geq\left(C+\frac{1}{2^{J}}\right) \sum_{k=1}^{J}\left|W_{\mu+k}\right|^{2}-c\left(\frac{1}{2^{\mu-1-J}}-\frac{1}{2^{\mu-1}}\right)\left|W^{s}\right|^{2} \\
& \quad-\left(C_{J}-C_{0}\right) \sum_{k=J+1}^{\mu}\left|W_{\mu+k}\right|^{2},
\end{aligned}
$$

holds for all $W \in \mathbb{C}^{m}$. We use the inequality (A.8) with $J=\mu-1$ in (A.7). This yields

$$
W^{*} S W \geq-c\left|W^{s}\right|^{2}+\left(C+\frac{1}{2^{\mu-1}}\right) \sum_{k=1}^{\mu-1}\left|W_{\mu+k}\right|^{2}+\left(s_{2 \mu}-C_{\mu-1}\right)\left|W_{2 \mu}\right|^{2} .
$$

It remains to choose $s_{2 \mu}:=C+C_{\mu-1}$ and the inequality

$$
W^{*} S W \geq-c\left|W^{s}\right|^{2}+C\left|W^{u}\right|^{2}
$$

holds.

\section{B. When does Kreiss' construction apply?}

In this appendix, we clarify when Kreiss' symmetrizer construction works. We show that it actually works only in case IV. We recall that we consider a matrix $M(\tau)$ that depends holomorphically on a complex variable $\tau \in \mathcal{V}$, where $\mathcal{V}$ is a neighborhood of 0 . Moreover, the expression of $M(\tau)$ is given, as in (5.1), by

$$
M(\tau)=i N+\left(\begin{array}{cccc}
b_{1}(\tau) & 0 & \cdots & 0 \\
\vdots & \vdots & & \vdots \\
b_{m}(\tau) & 0 & \cdots & 0
\end{array}\right), \quad N:=\left(\begin{array}{cccc}
0 & 1 & & 0 \\
\vdots & \ddots & \ddots & \\
\vdots & & \ddots & 1 \\
0 & \cdots & \cdots & 0
\end{array}\right) .
$$


Our result is the following.

Theorem B.1. Let $m \geq 2$ and let $M$ be given by (B.1) where the functions $b_{1}, \ldots, b_{m}$ are holomorphic on a neighborhood of 0 and vanish at 0 . Assume that there exists an integer $\mu \in\{1, \ldots, m-1\}$ such that the following property holds:

For all $K \geq 1$, there exist two constants $\alpha_{K}>0, c_{K}>0$, and there exists a $\mathcal{C}^{\infty}$ function $S_{K}$ defined on $]-\alpha_{K}, \alpha_{K}[\times]-\alpha_{K}, \alpha_{K}$ [ with values in $\mathcal{H}_{m}$ that satisfies

(i) for all $\delta \in]-\alpha_{K}, \alpha_{K}\left[\right.$ and all $\gamma \in\left[0, \alpha_{K}\left[, \operatorname{Re}\left(S_{K}(\gamma, \delta) M(\gamma+i \delta)\right) \geq\right.\right.$ $c_{K} \gamma I$

(ii) for all $\delta \in]-\alpha_{K}, \alpha_{K}\left[, S_{K}(0, \delta) \in \mathcal{S}_{m}\right.$,

(iii) for all $W \in \mathbb{C}^{m}, W^{*} S_{K}(0,0) W \geq K^{2}\left|W^{u}\right|^{2}-\left|W^{s}\right|^{2}$ with $W^{s}:=\left(W_{1}, \ldots, W_{\mu}\right)$ and $W^{u}:=\left(W_{\mu+1}, \ldots, W_{m}\right)$.

Then $M(i \delta)$ has purely imaginary coefficients for all $\delta \in \mathbb{R}$ sufficiently small.

Theorem B.1 shows that the choice $S_{K}(0, \delta) \in \mathcal{S}_{m}$ that was made in [9], see also [2,3], is convenient only in case IV. When the dissipation index is finite, it follows from Proposition 4.5, Proposition 6.1 or Proposition 7.1 that $M(i \delta)$ does not have purely imaginary coefficients for all small $\delta$. One therefore needs to consider a symmetrizer $S_{K}(0, \delta)$ with complex coefficients, that is a Hermitian matrix and not a real symmetric matrix. Thus our new construction of the symmetrizer in cases I, II, III was necessary.

Proof of Theorem B.1. We adopt some similar notation to what was introduced in the proof of Theorem 4.4. More precisely, we introduce the matrices $B_{q} \in \mathcal{M}_{m}(\mathbb{C})$ defined by

$$
\forall q \geq 1, \quad B_{q}:=\frac{i^{q-1}}{q !}\left(\begin{array}{cccc}
b_{1}^{(q)}(0) & 0 & \ldots & 0 \\
\vdots & \vdots & & \vdots \\
b_{m}^{(q)}(0) & 0 & \ldots & 0
\end{array}\right) \in \mathcal{M}_{m}(\mathbb{C}) .
$$

Using the holomorphy of $M$, we can expand $M(i \delta)$ as a power series, and get

$$
M(i \delta)=i N+i \sum_{q=1}^{+\infty} \delta^{q} B_{q} .
$$

In order to prove Theorem B.1, it is sufficient to show that all matrices $B_{q}$ have real coefficients. (The condition is not only sufficient but also necessary.) This property will be shown below by an induction argument on $q$.

For $K \geq 1$, we also introduce the notation $\mathbf{S}_{K}(\delta):=S_{K}(0, \delta)$, and

$$
\forall q \in \mathbb{N}, \quad \mathbf{S}_{q, K}:=\frac{1}{q !} \frac{\partial^{q} S_{K}}{\partial \delta^{q}}(0,0) \in \mathcal{S}_{m},
$$

where we have used the property (ii) of Theorem B.1. 
Let $K \geq 1$. Then $\mathbf{S}_{0, K}$ is a real symmetric matrix such that $\operatorname{Re}\left(i \mathbf{S}_{0, K} N\right)$ is nonnegative (use property (i) of Theorem B.1 at $\tau=0$ ). We feel the following Lemma can be used without proof since it is elementary.

Lemma B.2. Let $m \geq 2$ and let $H \in \mathcal{H}_{m}$ satisfy $H \geq 0$ and

$$
\forall j=2, \ldots, m, \quad H_{j, j}=0 .
$$

Then $H_{j, k}=0$ if $\max (j, k) \geq 2$. In particular if $H \in \mathcal{H}_{m} \cap i \mathcal{M}_{m}(\mathbb{R})$ satisfies $H \geq 0$, then $H=0$.

The matrix $\operatorname{Re}\left(i \mathbf{S}_{0, K} N\right)$ has purely imaginary coefficients, and we have seen that it is nonnegative. Lemma B.2 thus gives $\operatorname{Re}\left(i \mathbf{S}_{0, K} N\right)=0$. Applying Lemma 5.2, we find that $\mathbf{S}_{0, K}$ has the form (5.7), that is

$$
\mathbf{S}_{0, K}=\left(\begin{array}{cccc}
0 & \cdots & 0 & s_{1}^{K} \\
\vdots & 0 & s_{1}^{K} & s_{2}^{K} \\
0 & s_{1}^{K} & s_{2}^{K} & \vdots \\
s_{1}^{K} & s_{2}^{K} & \cdots & s_{m}^{K}
\end{array}\right) \in \mathcal{S}_{m}
$$

Let us show that the coefficient $s_{1}^{K}$ in (B.3) is non-zero. We use property (i) of Theorem B. 1 for $\delta=0$, and make a Taylor expansion for small $\gamma>0$. Using the relation $\operatorname{Re}\left(i \mathbf{S}_{0, K} N\right)=0$ that we have just found, the Taylor expansion gives

$$
\operatorname{Re}\left(S_{K}(0,0) M^{\prime}(0)+i \frac{\partial S_{K}}{\partial \gamma}(0,0) N\right) \geq c_{K} I .
$$

In particular, the upper left coefficient of $\operatorname{Re}\left(\mathbf{S}_{0, K} M^{\prime}(0)\right)$ is non-zero. In view of the form (B.3) of $\mathbf{S}_{0, K}$ and of the form (B.2) of $B_{1}=M^{\prime}(0)$, this yields $s_{1}^{K} \neq 0$.

Our goal is to show by induction on $q$ that the matrices $B_{q}$ in (B.2) have real coefficients. Let us first show that $B_{1}$ has real coefficients. We perform another Taylor expansion, but here we use property (i) with $\gamma=0$ and expand with respect to $\delta$. We obtain

$$
\operatorname{Re}\left(\mathbf{S}_{K}(\delta) M(i \delta)\right)=\delta \operatorname{Re}\left(i \mathbf{S}_{1, K} N+i \mathbf{S}_{0, K} B_{1}\right)+O\left(\delta^{2}\right) \geq 0 .
$$

The latter inequality implies

$$
\operatorname{Re}\left(i \mathbf{S}_{1, K} N+i \mathbf{S}_{0, K} B_{1}\right)=0,
$$

because $\delta$ can be either positive or negative. In particular, $\operatorname{Re}\left(i \mathbf{S}_{0, K} B_{1}\right)$ is a Hermitian matrix with purely imaginary coefficients. Using the form (B.3) of $\mathbf{S}_{0, K}$, with $s_{1}^{K} \neq 0$, together with the expression (B.2) of $B_{1}$, we obtain that all coefficients of $B_{1}$ are real. 
Showing that $B_{2}$ has real coefficients is a little more difficult. Expanding $M(i \delta)$ and $\mathbf{S}_{K}(\delta)$ at second order in $\delta$ and using property (i), we obtain

$$
\operatorname{Re}\left(i \mathbf{S}_{2, K} N+i \mathbf{S}_{1, K} B_{1}+i \mathbf{S}_{0, K} B_{2}\right) \geq 0
$$

Using the form (B.2) of $B_{2}$ and the form (B.3) of $\mathbf{S}_{0, K}$, the matrix $\operatorname{Re}\left(i \mathbf{S}_{0, K} B_{2}\right)$ reads

$$
\operatorname{Re}\left(i \mathbf{S}_{0, K} B_{2}\right)=\left(\begin{array}{cccc}
* & \ldots & \ldots & * \\
\vdots & 0 & \ldots & 0 \\
\vdots & \vdots & & \vdots \\
* & 0 & \ldots & 0
\end{array}\right)
$$

where the $*$ symbols denote some complex numbers. In particular, the $(j, j)$ element of the matrix $\operatorname{Re}\left(i \mathbf{S}_{0, K} B_{2}\right)$ vanishes for $j \geq 2$. We also know that the matrix $\operatorname{Re}\left(i \mathbf{S}_{2, K} N+i \mathbf{S}_{1, K} B_{1}\right)$ belongs to $\mathcal{H}_{m} \cap i \mathcal{M}_{m}(\mathbb{R})$ so its diagonal elements are zero. We can thus apply Lemma B.2 and derive

$$
\operatorname{Re}\left(i \mathbf{S}_{2, K} N+i \mathbf{S}_{1, K} B_{1}+i \mathbf{S}_{0, K} B_{2}\right)=\left(\begin{array}{cccc}
d & 0 & \ldots & 0 \\
0 & 0 & & \vdots \\
\vdots & & & \vdots \\
0 & \ldots & \ldots & 0
\end{array}\right), \quad d \geq 0
$$

The coefficient $d$ in (B.4) equals $-s_{1}^{K} \operatorname{Im} b_{m}^{\prime \prime}(0)$. If we can show that $d$ is zero, then the matrix $\operatorname{Re}\left(i \mathbf{S}_{0, K} B_{2}\right)$ will have purely imaginary coefficients. Consequently $B_{2}$ will have real coefficients (use again the form (B.3) of $\mathbf{S}_{0, K}$ ). Let us argue by contradiction and assume that $d$ is non-zero. In other words, let us assume that $b_{m}^{\prime \prime}(0)$ is not a real number (observe that this assumption is independent of $K$ ). The matrix in (B.4) is the sum of a matrix with purely imaginary coefficients and of $\operatorname{Re}\left(i \mathbf{S}_{0, K} B_{2}\right)$. For all $j=2, \ldots, m$, the coefficient $\operatorname{Re}\left(i \mathbf{S}_{0, K} B_{2}\right)_{1, j}$ is a purely imaginary number since it must compensate the $(1, j)$ element of $\operatorname{Re}\left(i \mathbf{S}_{2, K} N+\right.$ $\left.i \mathbf{S}_{1, K} B_{1}\right)$. We thus have

$$
\forall j=2, \ldots, m, \quad\left(\operatorname{Re}\left(i \mathbf{S}_{0, K} B_{2}\right)\right)_{1, j} \in i \mathbb{R}
$$

Using (B.2) and (B.3), these $m-1$ relations can be recast as a triangular linear system

$$
\left(\begin{array}{ccc}
\operatorname{Re}\left(i b_{m}^{\prime \prime}(0)\right) & & 0 \\
\vdots & \ddots & \\
\operatorname{Re}\left(i b_{2}^{\prime \prime}(0)\right) & \ldots & \operatorname{Re}\left(i b_{m}^{\prime \prime}(0)\right)
\end{array}\right)\left(\begin{array}{c}
s_{2}^{K} \\
\vdots \\
s_{m}^{K}
\end{array}\right)=-s_{1}^{K}\left(\begin{array}{c}
\operatorname{Re}\left(i b_{m-1}^{\prime \prime}(0)\right) \\
\vdots \\
\operatorname{Re}\left(i b_{1}^{\prime \prime}(0)\right)
\end{array}\right)
$$


In particular, there exists some real numbers $\sigma_{2}, \ldots, \sigma_{m}$ that can be computed from the matrix $B_{2}$ and are independent of $K$, such that

$$
\left(\begin{array}{c}
s_{2}^{K} \\
\vdots \\
s_{m}^{K}
\end{array}\right)=s_{1}^{K}\left(\begin{array}{c}
\sigma_{2} \\
\vdots \\
\sigma_{m}
\end{array}\right) .
$$

The expression (B.3) of $\mathbf{S}_{0, K}$ reduces to

$$
\mathbf{S}_{0, K}=s_{1}^{K}\left(\begin{array}{cccc}
0 & \cdots & 0 & 1 \\
\vdots & 0 & 1 & \sigma_{2} \\
0 & 1 & \sigma_{2} & \vdots \\
1 & \sigma_{2} & \cdots & \sigma_{m}
\end{array}\right)
$$

Let us now consider the vector $W:=\left(-\sigma_{m} / 2,0, \ldots, 0,1\right)$ that does not depend on $K$. We use the property (iii) of Theorem B.1 and derive

$$
0=W^{*} \mathbf{S}_{0, K} W \geq K^{2}\left|W^{u}\right|^{2}-\left|W^{s}\right|^{2}=K^{2}-\frac{\sigma_{m}^{2}}{4} .
$$

Since $K$ can be arbitrarily large and $\sigma_{m}$ is fixed, we are led to a contradiction. We have therefore obtained $d=0$ in (B.4). As explained before, this gives first $\operatorname{Re}\left(i \mathbf{S}_{0, K} B_{2}\right) \in \mathcal{H}_{m} \cap i \mathcal{M}_{m}(\mathbb{R})$, which implies $B_{2} \in \mathcal{M}_{m}(\mathbb{R})$ because of the form of $B_{2}$. We have also obtained the relations:

$$
\forall q=1,2, \quad \operatorname{Re}\left(i \mathbf{S}_{q, K} N+i \sum_{p=0}^{q-1} \mathbf{S}_{p, K} B_{q-p}\right)=0 .
$$

An induction argument then shows that for all integer $Q \geq 1$, the matrices $B_{1}, \ldots, B_{2} Q$ have real coefficients, and that the relations

$$
\forall q=1, \ldots, 2 Q, \quad \operatorname{Re}\left(i \mathbf{S}_{q, K} N+i \sum_{p=0}^{q-1} \mathbf{S}_{p, K} B_{q-p}\right)=0
$$

hold. Theorem B.1 is proved.

\section{References}

[1] H. BAUMgäRTEL, "Analytic Perturbation Theory for Matrices and Operators”, Birkhäuser Verlag, 1985.

[2] S. Benzoni-Gavage and D. Serre, "Multidimensional Hyperbolic Partial Differential Equations", Oxford Mathematical Monographs, Oxford University Press, 2007. 
[3] J. Chazarain and A. Piriou, "Introduction to the Theory of Linear Partial Differential Equations", North-Holland, 1982.

[4] J.-F. COulOMbel, Stability of finite difference schemes for hyperbolic initial boundary value problems, SIAM J. Numer. Anal. 47 (2009), 2844-2871.

[5] E. GodlewsKi and P.-A. Raviart, "Numerical Approximation of Hyperbolic Systems of Conservation Laws", Springer-Verlag, 1996.

[6] B. Gustafsson, H.-O. Kreiss and J. Oliger, "Time Dependent Problems and Difference Methods", John Wiley \& Sons, 1995.

[7] B. Gustafsson, H.-O. Kreiss and A. Sundström, Stability theory of difference approximations for mixed initial boundary value problems II, Math. Comp. 26 (1972), 649686.

[8] H.-O. KREISS, Stability theory for difference approximations of mixed initial boundary value problems, I, Math. Comp. 22 (1968), 703-714.

[9] H.-O. KREISS, Initial boundary value problems for hyperbolic systems, Comm. Pure Appl. Math. 23 (1970), 277-298.

[10] G. Métivier and K. Zumbrun, Symmetrizers and continuity of stable subspaces for parabolic-hyperbolic boundary value problems, Discrete Contin. Dyn. Syst. 11 (2004), $205-220$

[11] G. MÉTIVIER and K. ZUMBRUN, Hyperbolic boundary value problems for symmetric systems with variable multiplicities, J. Differential Equations 211 (2005), 61-134.

[12] D. Michelson, Stability theory of difference approximations for multidimensional initialboundary value problems, Math. Comp. 40 (1983), 1-45.

[13] R. Mneimné and F. Testard, "Introduction à la théorie des groupes de Lie classiques", Hermann, 1986.

[14] J. RAlston, Note on a paper of Kreiss, Comm. Pure Appl. Math. 24 (1971), 759-762.

[15] R. S AK Амото, Mixed problems for hyperbolic equations, I, Energy inequalities, J. Math. Kyoto Univ. 10 (1970), 349-373.

CNRS \& Université Lille 1

Laboratoire Paul Painlevé (UMR CNRS 8524)

and

Project Team SIMPAF of INRIA Lille

Nord Europe Cité scientifique

Bâtiment M2

59655 VILLENEUVE D’ASCQ Cedex, France jfcoulom@math.univ-lille1.fr 